\title{
Chiral Hypervalent, Pentacoordinated Phosphoranes
}

\author{
Dorota Krasowska $^{1, *, \dagger}$, Jacek Chrzanowski ${ }^{1, *, \dagger}$, Piotr Kiełbasiński ${ }^{1, \dagger}$ and Józef Drabowicz ${ }^{1,2, *, \dagger}$ \\ 1 Centre of Molecular and Macromolecular Studies, Polish Academy of Sciences, Sienkiewicza 112, \\ 90-363 Lodz, Poland; piokiel@cbmm.lodz.pl \\ 2 Department of Chemistry, Environment Protection and Biotechnology, \\ Jan Dlugosz University in Czestochowa, Armii Krajowej Ave. 13/15, 42-200 Czestochowa, Poland \\ * Correspondence: dkrasowska@gmail.com (D.K.); jacekchrzanowski1@gmail.com (J.C.); \\ drabow@gmail.com (J.D.); Tel.: +48-42-680-32-34 (J.D.) \\ + These authors contributed equally to this work.
}

Academic Editor: György Keglevich

Received: 29 September 2016; Accepted: 16 November 2016; Published: 21 November 2016

Abstract: This review presents synthetic procedures applied to the preparation of chiral (mainly optically active) pentacoordinated, hypervalent mono and bicyclic phosphoranes. The mechanisms of their stereoisomerization and their selected interconversions are also presented.

Keywords: hypervalency; chirality; phosphoranes; Berry pseudorotation; turnstile rotation

\section{Introduction}

The phenomenon of chirality plays a very important role in organic chemistry and biological processes [1-5]. Therefore an easy access to chiral compounds, especially optically active species, constitutes a vital challenge in modern synthetic organic chemistry. At least two reasons are responsible for this fact. Firstly, single stereoisomers are used very often as active components in formulations which serve as drugs, food additives or substances having the requested flavor or fragrance. The second reason stems from their application as chiral catalysts in various asymmetric reactions [6,7]. Moreover, detailed mechanistic description of a number of reactions would not be possible without taking into account their stereochemical aspects. These can be studied only with the use of optically active species which allow simultaneous application of kinetic and polarimetric measurements carried out with the use of the suitably designated model substrates (enantiomerically or diastereomerically pure or enriched samples) containing an appropriate chirality element [8]. In the organophosphorus chemistry the phenomenon of chirality is very common among organic derivatives having different valency and/or coordination number $[9,10]$. Their optical activity can be related to the presence of the following elements of chirality:

(a) a stereogenic center (for trivalent, tricoordinated, tetravalent tetracoordinated, pentavalent tetracoordinated derivatives)

(b) "trigonal bipyramidal chirality"

(c) "tetragonal bipyramidal chirality"

Organophosphorus derivatives in which optical activity results from the presence of a phosphorus atom at a center of trigonal bipyramid, are commonly named phosphoranes [11,12]. They have the general formula $\mathrm{P}\left(\mathrm{L}_{\mathrm{a}}\right)_{2}\left(\mathrm{~L}_{\mathrm{e}}\right)_{3}$, where " $\mathrm{a}$ " means an axial position and " $\mathrm{e}$ " means an equatorial position (Figure 1). 


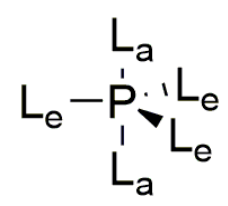

Figure 1. General formula of a phosphorus-containing trigonal bipyramid.

They constitute the family of hypervalent derivatives, showing "trigonal bipyramidal chirality" in which the central phosphorus atom has expanded its valence shell from 8 to 10 electrons (the concept was for the first time proposed by Musher in 1969) [13]. According to general systematic scheme proposed by Martin and coworkers [14,15] [N-P-L $\left({ }_{n} A_{m} B\right)$ coding system, in which N stands for the number of valence electrons associated formally with a central phosphorus atom and $L$ shows the number of ligands (A and B stand for the bonding element)] — this group should be considered as the family of 10-P-5 derivatives. The definition of hypervalency is also formally fulfilled by the corresponding phosphonium ylides (in which a phosphorus atom has also expanded its formal valence shell from 8 to 10 electrons). However, when one considers that the important resonance structures of these compounds are polarized from phosphorus to carbon it becomes evident that they represent 8-P-4 species (Figure 2). Therefore they do not meet the condition of hypervalency and they are excluded from this review.

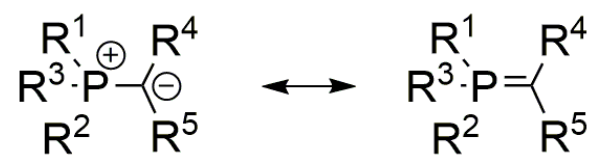

Figure 2. Resonance structure of phosphonium ylides.

The nature of bonding of hypervalent derivatives is presented in details in a few chapters of "The Chemistry of Hypervalent Compounds" [16,17] and more recently has again been studied using the topological analysis of the electron localization function (ELF)" [18]. Their structure and reactivity are dominated by the weak three-center-four-electron (3c-4e) bond. Its weakness results from the fact that two of the four electrons that participate in the bonding are in a nonbonding molecular orbital.

Phosphoranes, like other compounds having a trigonal bipyramide (TBP) geometry can exist in an enantiomeric form when the number of different ligands is sufficiently large to induce chirality of such a structure. For 10-P-5 derivatives having the general structure with five substituents (Figure 1) bonded to the central phosphorus atom the numbers of achiral and chiral structures are given in Table 1. According to the Mutterties rule [19] in hypervalent structures the more electronegative ligands tend to occupy the apical positions whereas the lone electron pair "should be located at the equatorial position" [19]. Moreover, "five- and six-membered rings, which stabilize the hypervalent molecules, span both the axial and equatorial positions" [14].

Table 1. Number of chiral and achiral species of 10-P-5 (TBP geometry).

\begin{tabular}{cc}
\hline General Structure & Number of Different Structures and Their Chirality \\
\hline $\mathrm{PL}_{5}$ & 1 achiral \\
$\mathrm{PL}_{4}^{1} \mathrm{~L}^{2}$ & 2 achiral \\
$\mathrm{PL}^{1}{ }_{3} \mathrm{~L}^{2}$ & 3 achiral \\
$\mathrm{PL}_{3}{ }_{3} \mathrm{~L}^{2} \mathrm{~L}^{3}$ & 2 achiral and 2 pairs of enantiomers \\
$\mathrm{PL}_{2} \mathrm{~L}_{2}{ }_{2} \mathrm{~L}^{3}$ & 2 achiral and 3 pairs of enantiomers \\
$\mathrm{PL}_{2}{ }_{2} \mathrm{~L}^{2} \mathrm{~L}^{3} \mathrm{~L}^{4}$ & 1 achiral and 6 pairs of enantiomers \\
$\mathrm{PL}^{1} \mathrm{~L}^{2} \mathrm{~L}^{3} \mathrm{~L}^{4} \mathrm{~L}^{5}$ & 10 pairs of enantiomers \\
\hline
\end{tabular}

It is evident from this table that phosphoranes containing at least three different ligands can be chiral and for structures $\mathrm{PL}_{2}{ }_{2} \mathrm{~L}_{2} \mathrm{~L}^{3}$ chirality may appear in the symmetric bicyclic spiro derivatives 
(Figure 3). Therefore, from the synthetic point of view, hypervalent structures exhibiting chirality due to the presence of one or two bidentate ligands should be more easily available than its acyclic analogues.

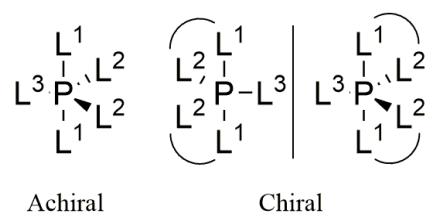

Figure 3. Chiral and achiral structure of hypervalent phosphoranes.

In hypervalent phosphoranes 10-P-5 the apical and equatorial ligands can be interchanged with each other according to the Berry pseudorotation mechanism (BPR) [20-22] (Scheme 1) or by the "turnstile" rotation mechanism (TR) proposed by Ugi and Ramirez [23-26] (Scheme 2). It should be noted here that the very recent DFT calculations of the fluxional behavior of experimentally known pentavalent molecules have suggested the equivalence of the turnstile rotation with the Berry pseudorotation. This suggestion is based on identification of three principal mechanisms by which the substituents interchange can be achieved (Berry pseudorotation, threefold cyclic permutation, and half-twist axial-equatorial interchange) [27].

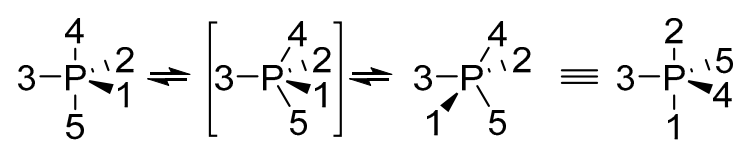

Scheme 1. Berry pseudorotation mechanism.

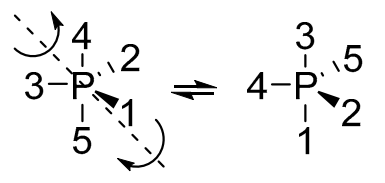

Scheme 2. "Turnstile" rotation mechanism (TR).

As early as 1949, Wittig and Rieber [28] reported isolation of pentaphenylphosphorane as the first example of a pentacoordinated organophosphorus derivative. However, a real opening in the hypervalent organophosphorus chemistry can be ascribed to a much later finding that the Ramirez reaction affords oxyphosphoranes by an oxidative addition of $\alpha$-diketones to trivalent phosphines [29,30] and Westheimer's proposal that a pentacoordinate phosphorane (able to stereomutate by pseudorotation) is involved in the hydrolysis of phosphoric esters [31]. Since that time, studies devoted to the synthesis, structural determinations, stereochemistry and reactivity of this group of the organophosphorus derivatives, have been carried out in a number of academic and industrial laboratories. Their results are regularly published in renowned chemical journals and, fortunately for those who work on these topics, are regularly presented in the chapters devoted to penta- and hexacoordinated organophosphorus derivatives included in the subsequent volumes of RSC monographic series "Organophosphorus Chemistry" [32-43]. In this respect, a very recent review highlighting methods for the asymmetric synthesis of P-chiral pentacoordinated spirophosphoranes should also be mentioned [44].

\section{Chiral Hypervalent Phosphoranes and Their Anions}

\subsection{Chiral Phosphoranes as Reactive Intermediates}

In most cases the papers describing the involvement of unstable chiral phosphoranes as reactive intermediates tackle the problem of the mechanism of $S_{N^{-}}-\mathrm{P}$ reactions. This is connected with the fact 
that a nucleophilic substitution at phosphorus can occur either synchronously according to an $\mathrm{S}_{\mathrm{N}} 2-\mathrm{P}$ mechanism or in a stepwise manner by an addition-elimination mechanism (A-E). The later involves formation of a phosphorane as an intermediate, by the addition of a nucleophile $\mathrm{s}(\mathrm{Nu})$ to a chiral substrate. It is now generally accepted that diaxial or diequatorial disposal of entering " $\mathrm{Nu}^{\prime}$ " and leaving " $\mathrm{L}$ " groups in a trigonal bipyramidal structure of a transient or intermediary phosphorane should lead to inversion of the configuration at phosphorus while the steric course of axial-equatorial substitution (which is possible if a phosphorane intermediate undergoes pseudorotation) is predicted to be a retention (Scheme 3).

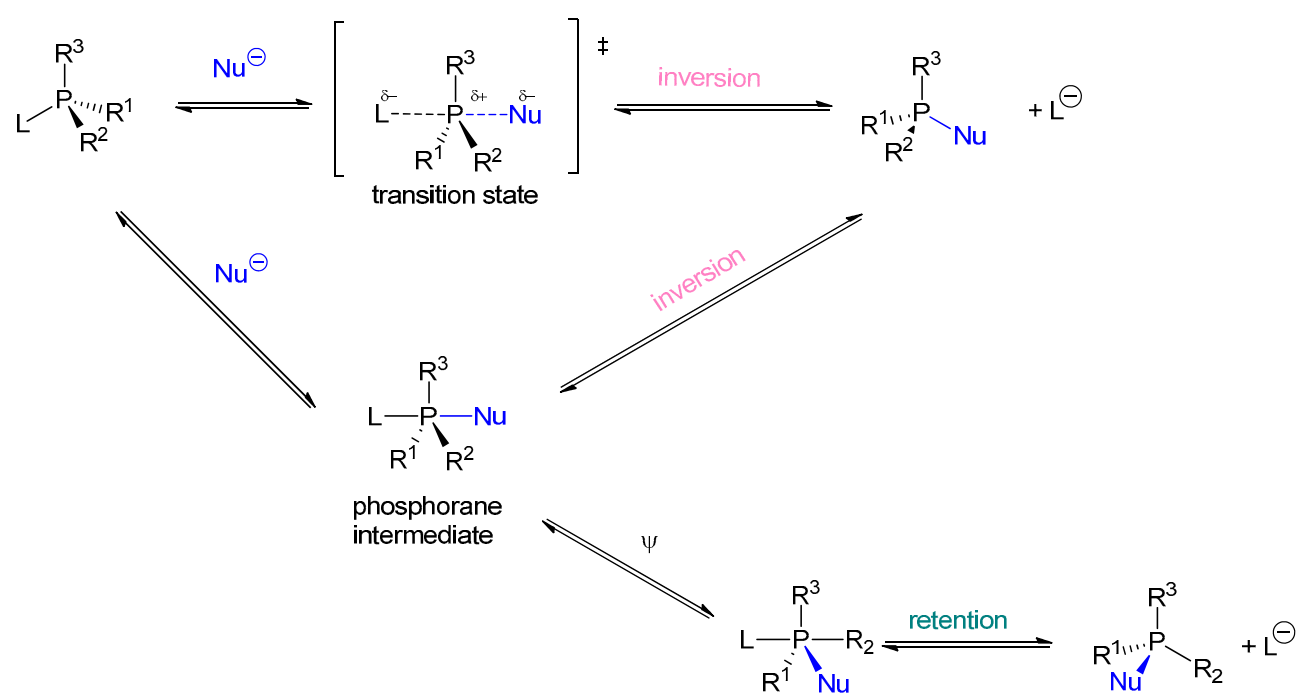

Scheme 3. Possible mechanism for nucleophilic substitution reaction at phosphorus.

The stereochemical consequences (retention or inversion) of such substitution reactions may be conveniently discussed with the aid of the Desargues-Levi graph [45], which originally was proposed by Mislow [46] for stereoisomerization of pentacoordinate phosphoranes.

The participation of such chiral phosphoranes $\mathbf{I}-\mathbf{V}$ as intermediates and their pseudorotation was suggested by Juge and coworkers [47] to explain retention of configuration during the rearrangement of the lithium salts $\mathbf{2 a - c}$ derived from the 2-bromophenylphosphinite boranes $\mathbf{1 a}-\mathbf{c}$ to the $o$-hydroxyphenylphosphine boranes 3a-c induced by halogen-metal exchange (Scheme 4) [47].

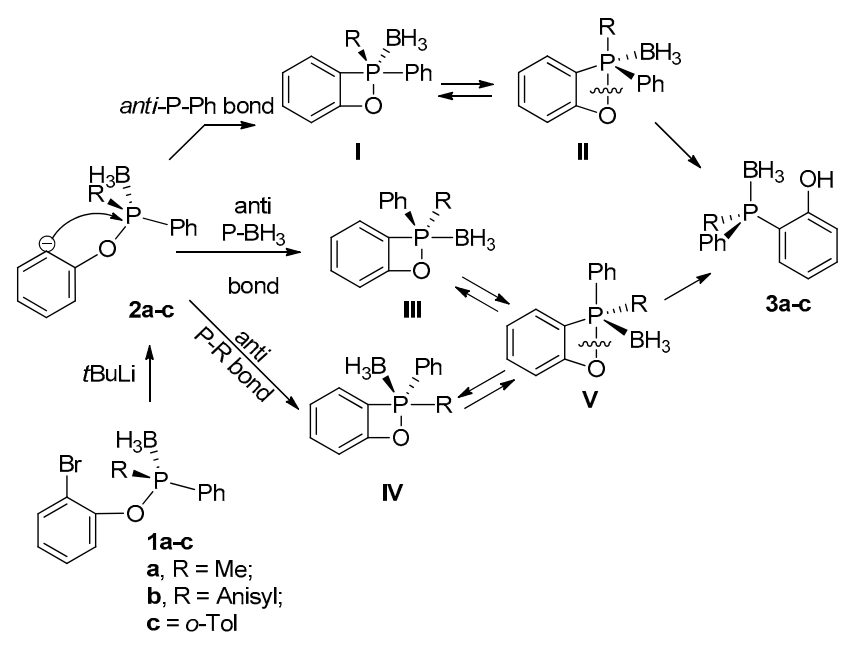

Scheme 4. The rearrangement of the lithium salts $2 a-c$ derived from the 2-bromophenylphosphinite boranes $\mathbf{1 a}-\mathbf{c}$ to the $o$-hydroxyphenylphosphine boranes $3 \mathbf{a}-\mathbf{c}$. 
Earlier the same group proposed that pseudorotation of the intermediate phosphoranes VI, VII was responsible for predominant retention of configuration during the regiospecific ring opening reaction of the diastereomerically pure dioxaphospholane borane 4 with organolithium reagents which afforded phosphinite boranes $\mathbf{7 a}, \mathbf{b}$ (Scheme 5) [48].
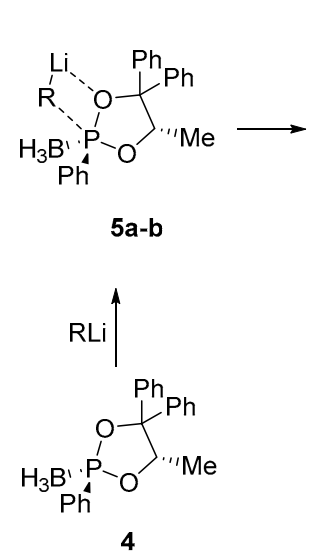

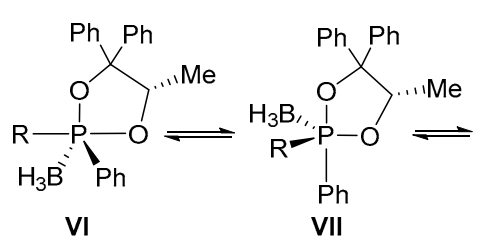

VII

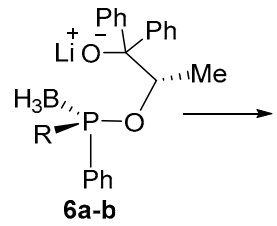

6a-b

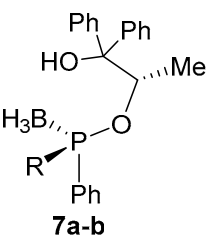

a; $\mathrm{R}=\mathrm{Me}$

b; $R=o-A n$

Scheme 5. Stereochemical outcome of the regiospecific ring opening reaction of the diastereomerically pure dioxaphospholane borane 4 with organolithium reagents.

As another example of such a transformation, hydrolysis of quasiphosphonium salts, cis- and trans-3-methoxy-2,2,6-trimethyl-3-phenyl-1,3-oxaphosphorinanium tetrafluoroborate salts $\mathbf{8 a}$ and $\mathbf{8 b}$ can be presented, by which the corresponding phosphine oxides $\mathbf{9 a}$ and $\mathbf{9 b}$ were formed with complete retention of configuration at phosphorus. The detailed NMR and stereochemical studies allow rationalization of the stereochemical outcome of this reaction in terms of the addition-elimination mechanism, A-E, involving two hydroxyphosphorane intermediates 10a and $\mathbf{1 0 b}$ (that are able to undergo pseudorotation) in which at least one oxygen is apical as shown in Scheme 6 [49].
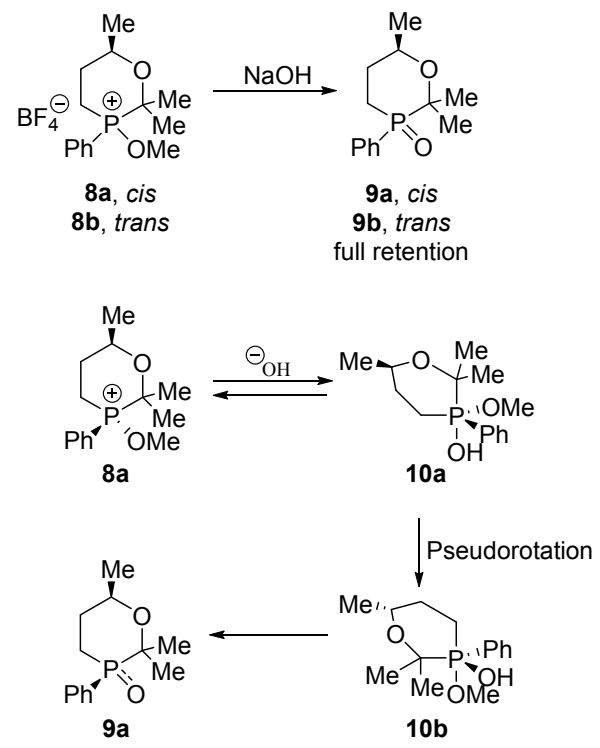

Scheme 6 . The hydrolysis of quasiphosphonium salts $8 \mathbf{a}$ and $8 \mathbf{b}$ involving two hydroxyphosphorane intermediates $\mathbf{1 0 a}$ and $\mathbf{1 0 b}$.

Similarly, the participation of the chiral hydridophosphorane $\mathbf{1 2}$ as an intermediate in the intramolecular transesterification of the 2-hydroxyphenyl phosphite $\mathbf{1 1}$ to the 3-hydroxypropyl phosphite 13 (Scheme 7) was supported by low temperature ${ }^{31}$ P-NMR measurements [50]. 


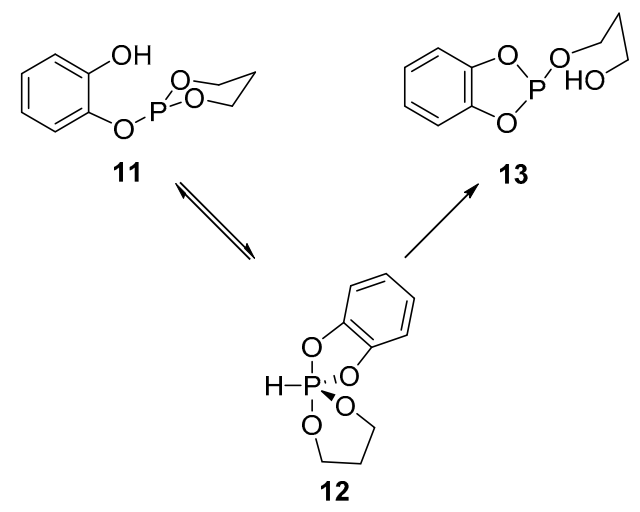

Scheme 7. The intramolecular transesterification of the 2-hydroxyphenyl phosphite 11.

Earlier, the participation of chiral hydridophosphoranes was proposed in the intermolecular transesterification of chiral asymmetric phenyl phosphites [51] and in the addition of achiral alcohols to compounds having a stereogenic tricoordinated phosphorus atom [52] and very recently in the hydrolysis of a trinucleoside monophosphate by the intramolecular $2 \alpha$-hydroxy group neighbouring the scissile phosphodiester linkage [53]. The regioselective formation of chiral 3-hydroxypropylphosphinates $\mathbf{1 7}$ from cyclic oxaphospholane $\mathbf{1 4}$ and Grignard reagents was explained by assuming that during the reactions a pentavalent TBP phosphorane intermediate $\mathbf{1 5}$ is formed, which could not undergo pseudorotation to generate another phosphorane $\mathbf{1 6}$ due to a high energetic barrier. Therefore, the endocyclic $\mathrm{P}-\mathrm{O}$ bond in $\mathbf{1 5}$ is cleaved almost exclusively to form the phosphinates 17 in high yields (Scheme 8) [54].

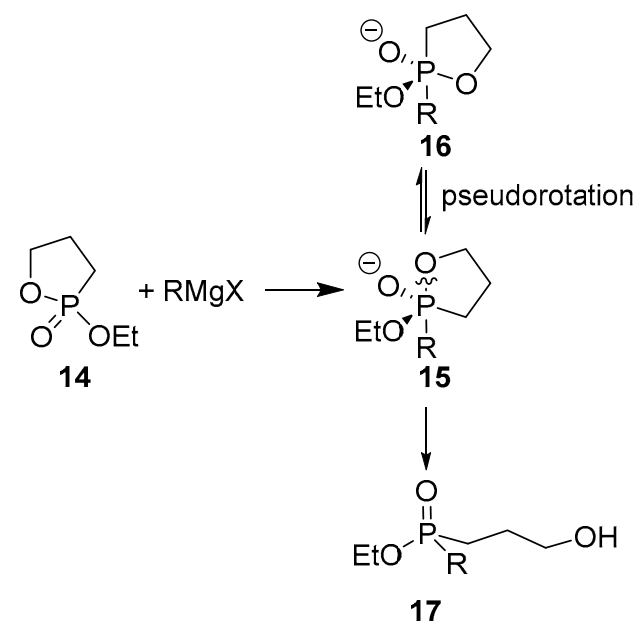

Scheme 8. Reaction of cyclic oxaphospholane 14 and Grignard reagents.

\subsection{Chiral Phosphoranes and Their Anions as Isolable Species}

As it was mentioned above, from the synthetic point of view, hypervalent structures exhibiting chirality due to the presence of one or two bidentate ligands should be more easily available than their acyclic analogues. Therefore, the majority of isolated hypervalent phosphoranes constitute monoand especially bicylic derivatives in which a cyclic frame include pentacoordinated phosphorus atom bonded to a carbon and/or heteroatoms.

\subsubsection{Chiral Monocyclic Phosphoranes and Their Anions as Isolable Species}

The non-concerted [2 + 2] cycloaddition reaction of P-haloylides 18 with trifluorometyl ketones led to the stereoselective formation of monocyclic halophosphoranes 19 (Scheme 9) [55]. 


$$
\mathrm{R}^{1} \mathrm{R}^{2} \mathrm{P}(\mathrm{Cl})=\mathrm{CHR}^{3}+\mathrm{R}^{4}\left(\mathrm{CF}_{3}\right) \mathrm{C}=\mathrm{O} \longrightarrow \mathrm{R}_{18}
$$

Scheme 9. Monocyclic halophosphoranes 19.

The monocyclic vinyl phosphoranes $\mathbf{2 1}$ were prepared by the reaction of cyclic phosphite $\mathbf{2 0}$ with dimethyl acetylenedicarboxylate in the presence of the appropriate phenol (Scheme 10) [56].

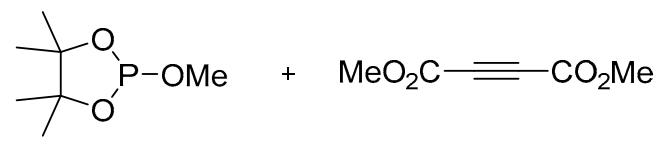

20

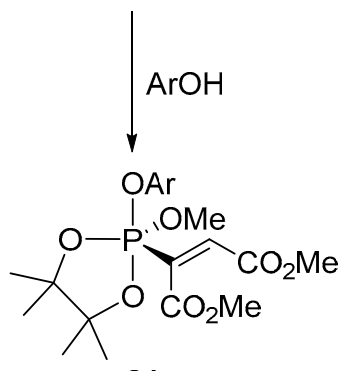

21

Scheme 10. Reaction of cyclic phosphite 20 with dimethyl acetylenedicarboxylate.

The formation of monocyclic phosphorane $\mathbf{2 3}$ was observed in the reaction of ylide $\mathbf{2 2}$ with hexafluoroacetone (Scheme 11) [57].

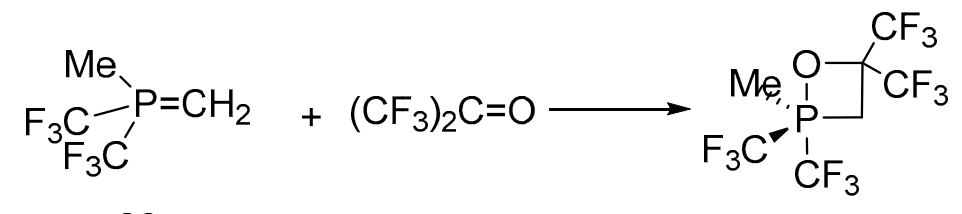

22

23

Scheme 11. Reaction of ylide 22 with hexafluoroacetone.

A similar reaction of ylide 24 with hexafluoroacetone gave monocyclic phosphorene 25 (Scheme 12) [57].

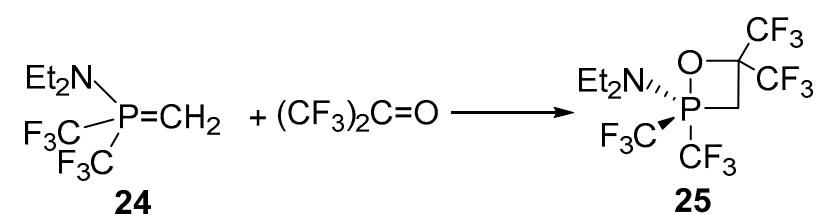

Scheme 12. Reaction of ylide 24 with hexafluoroacetone. 
The tricoordinate organophosphorus derivatives $\mathbf{2 6 a}-\mathbf{c}$ were found to react with hexafluoroacetone to give chiral monocyclic phosphoranes $\mathbf{2 7 a - c}$ (Scheme 13) in which no ligand exchange processes were observed at room temperature. However, the chiral phosphonic acid ester 29 was isolated upon hydrolysis of the chlorophosphorane $\mathbf{2 7 a}$, which proceeds most probably via hydroxyphosphorane $\mathbf{2 8}$ (Scheme 13) [58].

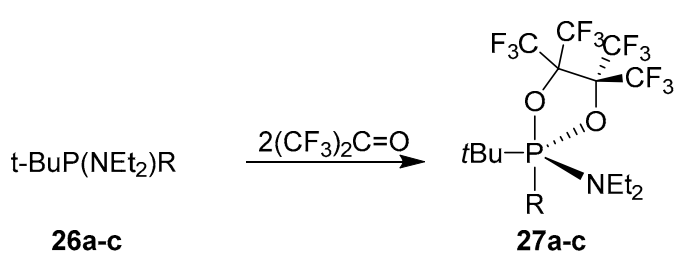
a, $\mathrm{R}=\mathrm{Cl}$
b, $\mathrm{R}=\mathrm{Br}$
c, $\mathrm{R}=\mathrm{OCH}\left(\mathrm{CF}_{3}\right)_{2}$<smiles>CCN(CC)C(C)(C)C(F)(F)F</smiles>

29
Hydrolysis for 27 a

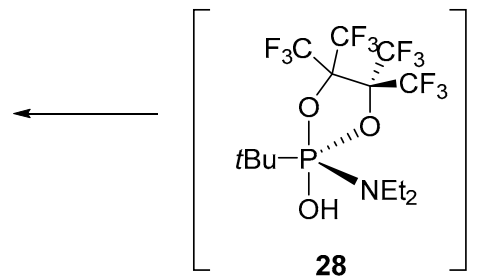

Scheme 13. Reaction of organophosphorus derivatives 26a-c with hexafluoroacetone.

Preparation of monocyclic halophosphoranes $31 \mathbf{a}, \mathbf{b}$ and $31 \mathbf{c}$ was based on the reaction of the tricoordinate phosphinites $\mathbf{3 0 a}, \mathbf{b}$ with benzyl chloride or benzyl bromide. The corresponding fluorophosphoranes 31d or 31e were isolated upon the chloride-fluoride or bromide-fluoride exchange reactions when the chloride 31a or bromide 31c were used as a substrate respectively (Scheme 14) [59].
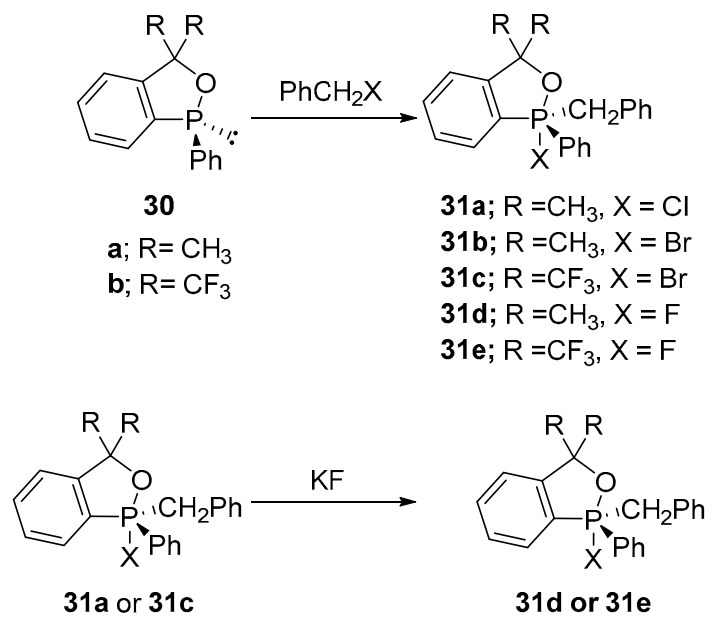

Scheme 14. Preparation of monocyclic halophosphoranes 31a-e.

Monocyclic (ethylthio)fluorophosphorane 34 was isolated in quantitative yield upon fluoride anion abstraction from $\mathrm{BF}_{4}$ counter anion of phosphonium salt 33 easily generated by the reaction of cyclic phosphinothionate 32 with triethyloxonium tetrafluoroborate. Defluorination of the fluorophosphorane 34 with trimethylsilyl trifluoromethanesulfonate gave phosphonium salt 35 [60] (Scheme 15). 


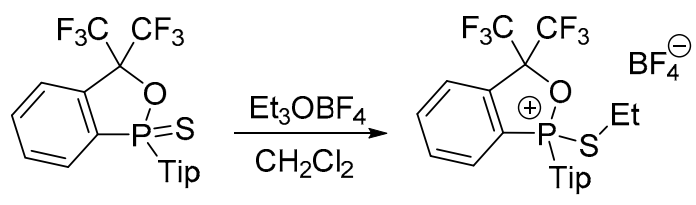

32

33

Tip= 2,4,6-triisopropylphenyl

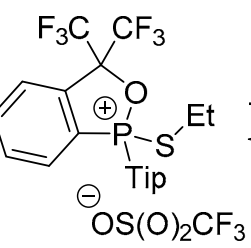

35

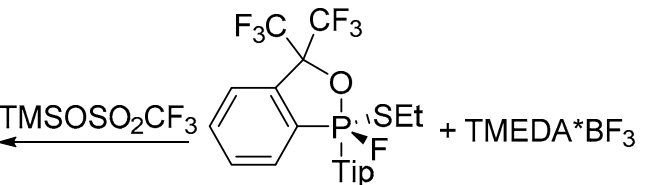

Tip

34

Scheme 15. Preparation of monocyclic (ethylthio)fluorophosphorane 34 .

\subsubsection{Chiral Bicyclic Phosphoranes and Their Anions as Isolable Species}

A relatively rich number of isolable bicyclic hypervalent phosphoranes have been well documented in the older and more recent chapters by Hellwinkel [61], Burger [62], Holmes [63], Husband and McNab [64], Burgada and Setton [65], Akiba [16], and Kawashima [66]. These chapters mention, more or less extensively, chiral bicyclic phosphoranes, but without deeper comments devoted to the phenomenon of chirality in such derivatives. Therefore, in this part of our compilation we are going to present, as comprehensively as possible, isolable hypervalent derivatives in which their chirality results from the presence of two heterocyclic units forming a P-spiro system. They are divided in accord with the commonly accepted Martin's N-P-L $\left({ }_{n} A_{m} B\right)$ coding system, in which N stands for the number of valence electrons associated formally with a central phosphorus atom and $L$ shows the number of ligands (A and B stand for the bonding element) [14].

\section{P-5C Phosphoranes}

Hellwinkel as early as 1966 reported on the isolation of the first optically active pentaarylphosphorane. Initially, hexacoordinate phosphorane potassium salt $\mathbf{3 7}$ was synthesized in good yield in racemic form by the reaction of bis(biphenyl)phosphonium iodide 36 with 2,2'-dilithio4-methyl-biphenyl. The optical resolution of the racemic salt 37 was based on its conversion into two diastereomeric ammonium salts by the treatment with $\mathrm{N}$-methylammonium iodide derived from brucine. The isolation of the levorotatory diastereoisomer $\left([\alpha]_{578}=-1200\right)$ was achieved in moderate yield by recrystallization of a crude crystalline fraction from acetone. The dextrorotatory diastereoisomer of the ammonium salt was isolated from a mother liquor in poor yield and with a lower optical purity $\left([\alpha]_{578}=+986\right)$. The isolated diastereoisomers were reconverted into the enantiomeric potassium salts upon the treatment with potassium iodide in acetone followed by repeating crystallizations. Their maximum specific rotation reached values $[\alpha]_{578}=+$ and -1870 . Acidification of optically active potassium salts of hexacoordinate phosphorus derivatives $(+)$ and (-)-37 with $\mathrm{HCl}$ in methanol/acetone solution provided the mixture of spirophosphoranes from which enantiomeric spirophosphoranes 38 having $[\alpha]_{578}=+$ and -94 , respectively were isolated by multiple recrystallization (Scheme 16). Additionally, the structure of 38 as a racemate was confirmed by the alternative method for its preparation using biphenyl-biphenylyl-2-phosphine $\mathbf{3 9}$ as the starting material (Scheme 17) [67]. 


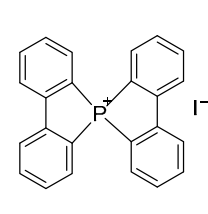

36

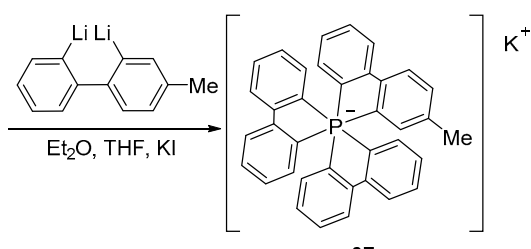

rac-37

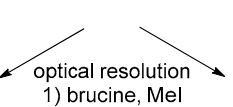

1) brucine, Mel

2) $\mathrm{KI}$ /acetone
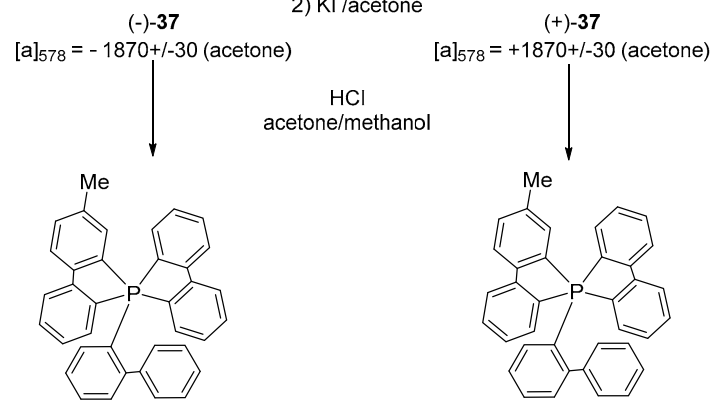

$(-)-38$

$[\alpha]_{578}=-94(\mathrm{THF})$

+ isomeric mixture

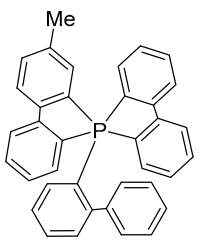

$(+)-39$

$[\alpha]_{578}=+94$ (THF)

+ isomeric mixture

Scheme 16. Preparation of enantiomeric spirophosphoranes 38.

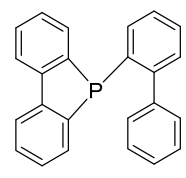

39

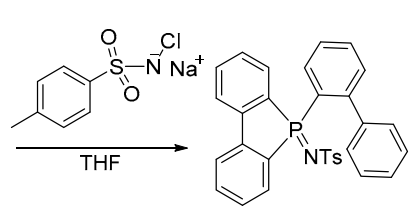

not isolated $\mathbf{4 0}$

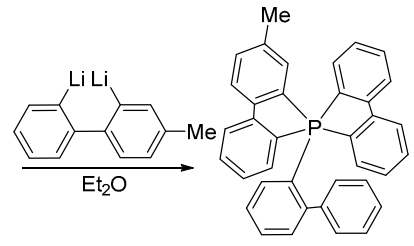

$38(85 \%)$

Scheme 17. Preparation of 38 using biphenyl-biphenylyl-2-phosphine 39.

More recently the reaction of spirocyclotetraalkylphosphonium salts 41 with a series of organolithium reagents RLi, was found to give spirobicyclophosphoranes $42 a-e$ in good to low yields (Scheme 18). Single crystal X-ray analysis of 42a showed a TBP geometry with the axial-equatorial rings and the methyl group in an equatorial position. Low temperature NMR measurements indicated that all the pentacoordinate structures $\mathbf{4 2}$ show fluxional behaviour in a solution with a very low pseudorotation barrier [68].

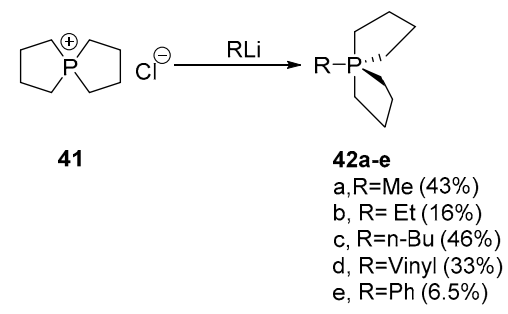

Scheme 18. Reaction of spirocyclotetraalkylphosphonium salts 41 with a series of organolithium reagents.

\section{P-4C-1O Phosphoranes}

Oxaphosphetane (OPA) is a widely recognized intermediate formed via an irreversible [2 + 2] cycloaddition of an aldehyde or ketone with the phosphonium ylide in the Wittig reactions. 
The stereoselectivity of the reaction is decided by the course of the first irreversible step. Then, the oxaphosphetane undergoes irreversible [2 + 2] syn cycloreversion to give an alkene and phosphine oxide. In most cases the diastereomeric ratio of the oxaphosphetane (OPA) intermediate exactly corresponds to the final alkene, except the cases when a stereochemical drift occurs in the formation of the alkene product. The process "stereochemical drift" refers to the nonstereospecific decomposition of the oxaphosphetane (OPA) intermediate in reactions of certain alkylides with certain aldehydes. The particular investigations on the stereochemical drift in the Wittig reactions (alkylidenephosphonium ylide with aldehydes) under Li-salt-free conditions were undertaken by D. G. Gilheany et al. [69], and based on Variable-Temperature NMR measurements. A particularly suitable candidate for the study of stereochemical drift was intermediate 44a formed in the reaction of biphenylphenylethylidene-phosphonium ylide $\mathbf{4 3}$ with 2-bromobenzaldehyde. It is due to its stability at room temperature (Scheme 19). It undergoes significant stereochemical drift on decomposition in THF at reflux: A sample containing cis- and trans-44a in a ratio of 94:6 gave alkenes of a $(Z) /(E)$ ratio $82: 18$ under reflux conditions. On the other hand, the decomposition conducted at $50{ }^{\circ} \mathrm{C}$ was not complete after $6 \mathrm{~d}$, but the maximum possible $(Z) /(E)$ ratio that could have been achieved in this reaction was 88:12, revealing that a smaller amount of the stereochemical drift had occurred. A very similar experiment supported with VTNMR spectra analysis over the temperature range -20 to $+40{ }^{\circ} \mathrm{C}$ on $44 \mathrm{~b}$ (produced in the reaction of biphenylphenylethylidenephosphonium ylide 43 and benzaldehyde) was performed. Oxaphosphetane intermediate $44 \mathrm{~b}$ was formed in a diastereomeric ratio 71:29 (cis/trans). Again, the ratio of OPA, ylide and phosphine oxide was invariant within the temperature range from -20 to $30^{\circ} \mathrm{C}$, which indicates that no decomposition to alkene and phosphine oxide occurred. On heating the OPA $44 \mathbf{b}$ was decomposed to produce the alkene in 53:47 (Z)/(E) isomeric ratio. In the ${ }^{31} \mathrm{P}$ NMR spectra recorded for $44 \mathbf{a}$ and $44 \mathbf{b}$ in the presence of an excess aldehyde additional signals appeared in the pentavalent region $(\delta=-50$ to $-80 \mathrm{ppm})$. The observation was consistent with that reported earlier by Vedejs et al. [70]. Thus, the apparent interaction between OPA and aldehyde was expected to have an impact on the occurrence of the stereochemical drift.

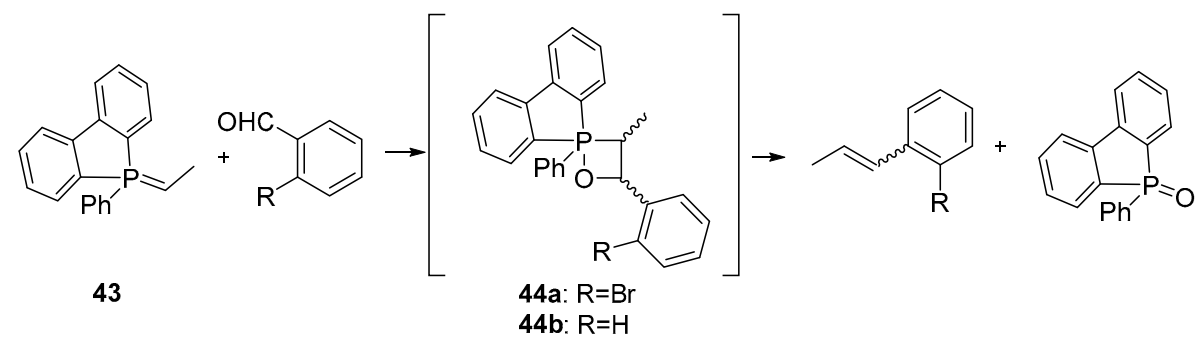

Scheme 19. Reaction of biphenylphenylethylidene-phosphonium ylide 43 with benzaldehydes.

\section{P-3C-1N-1O Phosphoranes}

In 1996 Kawashima and Okazaki succeeded in preparing pentacoordinate $\mathrm{N}$-apical 1,2-azaphosphetidines, whose structures were fully characterized. They also investigated their thermolysis. The first observation on the occurrence of the $N$-equatorial pseudorotamers was also reported [71]. The synthetic pathway was as follows: the treatment of 2-(methylphenylphosphinyl)- $\alpha$, $\alpha$-bis(trifluoromethyl)phenylmethanol 45 with $n$-BuLi led to the generation of methylene carbanion species, which subsequently underwent nucleophilic addition to a Schiff base providing the $\beta$-aminoethyl derivative 46. 1,2-Azaphosphetidine 47 was formed in 93\% yield by intramolecular cyclization followed by dehydration under the Mitsunobu reaction condition (Scheme 20). 

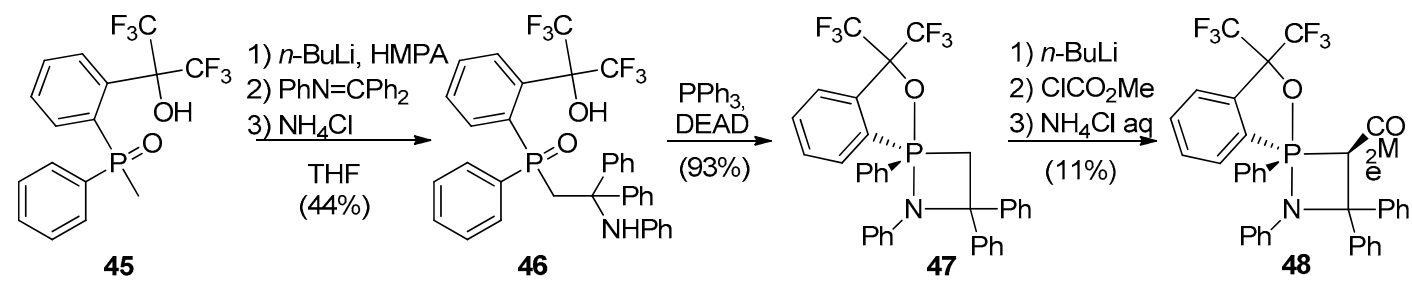

Scheme 20. Synthesis of 1,2-Azaphosphetidine 47 and 48.

A typical chemical shift in the NMR spectra was observed for compounds with a TBP geometry in which an apical position was occupied with $\mathrm{O}$ or $\mathrm{N}$ atom. X-ray single crystal analysis revealed the distorted TBP geometry with phosphorus as a central core. Hydrolysis of $\mathbf{4 7}$ on silica gel resulted in its cycloreversion to the starting material 46. Finally, 1,2-azaphosphetidine 47 was converted into the 3-methoxycarbonyl derivative 48, likewise it had been reported, for the corresponding $1,2-\lambda^{5}$-oxaphosphetanes [72,73]. In the ${ }^{31} \mathrm{P}-\mathrm{NMR}$ spectrum recorded for the solution of $\mathbf{4 7}$ in deuterated toluene, apart from the appropriate resonance signal $\left(\delta_{P}=-29.9\right)$, another upfielded peak $\left(\delta_{P}=-50.9\right)$, was observed which was assigned to the $N$-equatorial pseudorotamer 49 (Scheme 21). A similar pseudorotamer $\left(\delta_{\mathrm{P}}=-51\right)$ was observed also in the case of 48 .
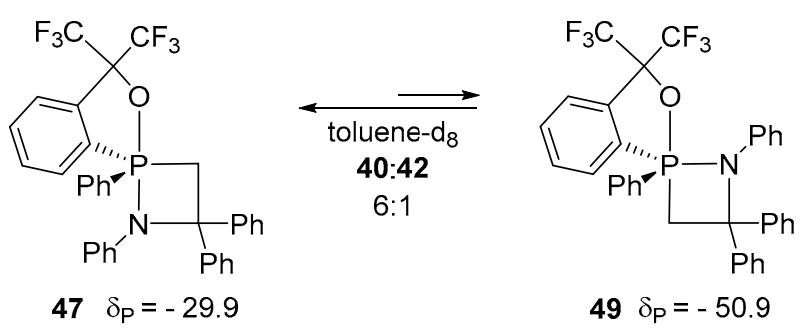

Scheme 21. The equilibrium between phosphoranes 47 and 49 .

Thermolysis of $\mathbf{4 7}$ or $\mathbf{4 8}$ proceeded with the quantitative formation of the corresponding alkenes and iminophosphorane (Scheme 22), which implies that azaphosphetidines should be considered as aza-Wittig intermediates.

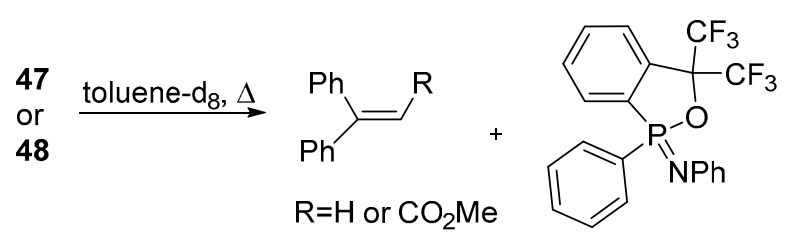

Scheme 22. Thermolysis of 47 or 48 .

The reaction of an iminophosphorane, the nitrogen analogue of a phosphonium ylide, with a reactive alkyne has been intensely studied because it constitutes a useful synthetic pathway for the corresponding $\alpha$-iminoalkylidenephosphoranes via a [2+2]-cycloadduct. The intermediate had neither been observed nor isolated until the first stable pentacoordinate $1,2-\lambda^{5}$-azaphosphetine $\mathbf{5 1}$ was synthesized by Kawashima et al. [74]. Its preparation was achieved by the cycloaddition reaction of the Martin ligand-based iminophosphorane $\mathbf{5 0}$ with an alkyne (Scheme 23). X-ray crystallography of 51 showed a distorted TBP with $\mathrm{N}$ and $\mathrm{O}$ atoms at the apical positions. The variable temperature ${ }^{31} \mathrm{P}$ NMR spectra of 51 in $C_{7} D_{8}$ or $C_{3} C N$ showed a shift to a lower field with decreasing temperature indicating that (51) was in an equilibrium with the corresponding ylide structure 52. 


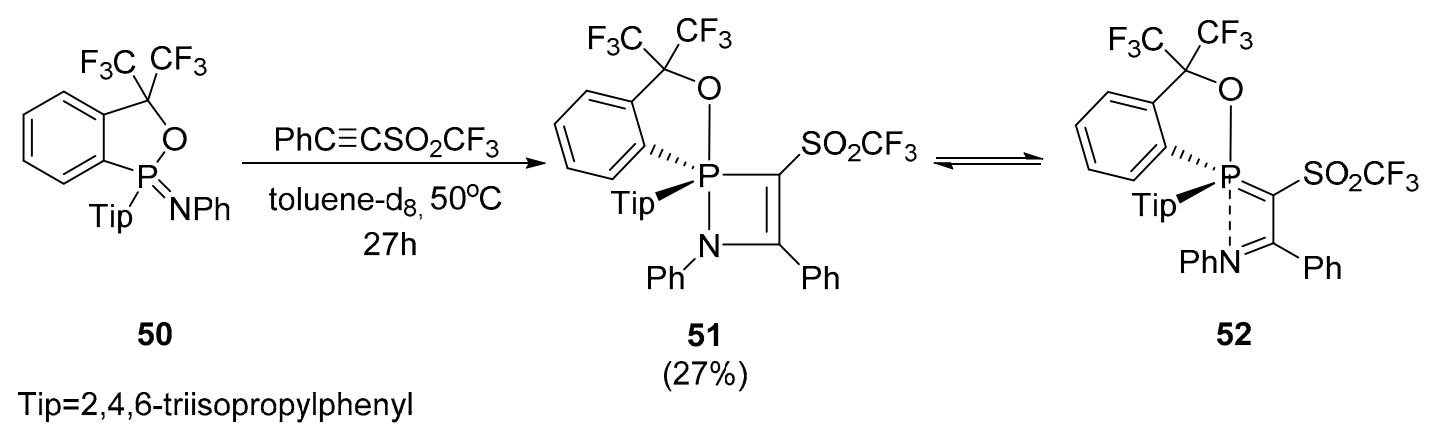

Scheme 23. Cycloaddition reaction of the iminophosphorane $\mathbf{5 0}$ with an alkyne.

The isomerization and thermal decomposition of isolable spiro-1,2-oxaphosphetanes has been studied in details with the aim to recognize a stereomutation mechanism of spiro-1,2-oxaphosphetanes, the Wittig intermediate products. Spiro-oxaphosphetanes $54-58$, stabilized by the presence of the $o$-benzamide moiety, were found to be good candidates for the studies [75]. The OPA's 54-56 were obtained according to the previously reported procedure [76] (Scheme 24). The reactions with the hindered ketone, L-(-)-camphor, gave products 57 and 58 (in a 45:55 ratio) arising from the endo attack at the $\mathrm{CO}$ group of L-camphor.

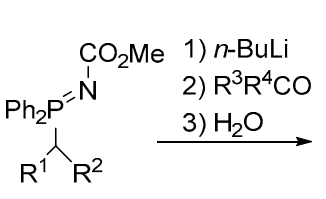

53

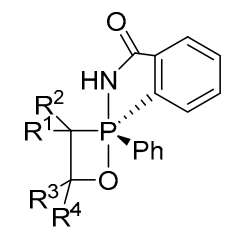

$54,55,56$<smiles>[R7]P1([R7])([R7])NC(=O)c2ccccc21</smiles>

$55 a, 56 a$

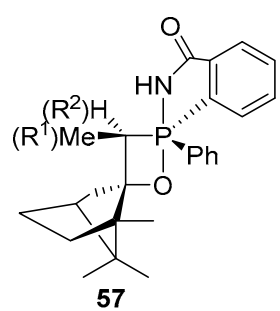

$\| \Delta$

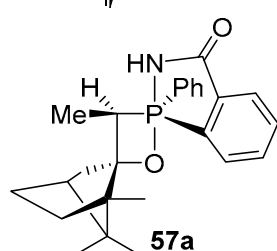

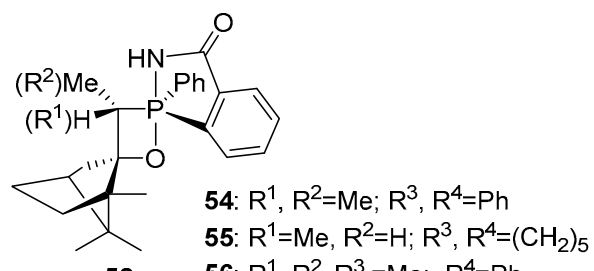

58
$=\left(\mathrm{CH}_{2}\right)_{5}$

57: $\mathrm{R}^{1}=\mathrm{Me}, \mathrm{R}^{2}=\mathrm{H}$;

58: $R^{1}=H, R^{2}=M e$

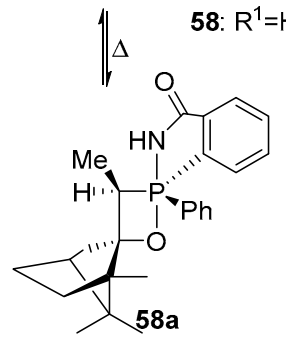<smiles>[R]C=C([R])[R]</smiles>

Scheme 24. Synthesis of spiro-oxaphosphetanes 54-58 and their thermal decompositions.

All thermal decompositions of OPAs 54-58 furnished the appropriate alkenes and a phosphinamide, quantitatively. On the thermolysis of 55-58, partial isomerization to 55a-58a was observed, which resulted in the inverted configuration at phosphorus atom. It has been found that oxaphosphetane decomposition took place in a single step via a polar transition state. For the first time the stereomutation through three possible mechanisms $\mathrm{MB}_{2}, \mathrm{MB}_{3}$, and $\mathrm{MB}_{4}$ involving two, three, and four Berry pseudorotations (at phosphorus atom), respectively, supported by DFT calculations has been evidenced [75]. 


\section{P-3C-2O Phosphoranes}

The first configurationally stable enantiomeric pair of 10-P-3C-2O phosphoranes with well-defined sole stereogenic centre at phosphorus atom was successfully obtained and characterized by Akiba et al. [77]. The synthesis of diastereomeric $60-\left(R_{P}\right)$ and $60-\left(S_{P}\right)$ was achieved via a facile alkylation of the in situ generated phosphoranide anion by (-)-menthyl chloroacetate in $87 \%$ yield (Scheme 25). The diastereomeric mixture could be resolved effectively by fractional crystallization to furnish $60-\left(R_{\mathrm{P}}\right)$ in $25 \%$ and $60-\left(S_{\mathrm{P}}\right)$ in $22 \%$ yield. Thus, the separation and the selection of suitable crystals allowed for the determination of absolute configurations for both diastereoisomers by $\mathrm{X}$-ray diffraction. The alkylation of $\mathrm{P}-\mathrm{H}$ phosphorane derivatives was recognized to proceed with complete retention of configuration at the phosphorus sterereogenic centre, most probably due to no detectable racemization at the step of phosphoranide intermediate formation.<smiles>FC(F)(F)c1ccccc1[PH](OC(F)(F)C(F)(F)F)(c1ccccc1C(F)(F)F)C(F)(F)F</smiles>

(rac) $-\mathbf{5 9}$

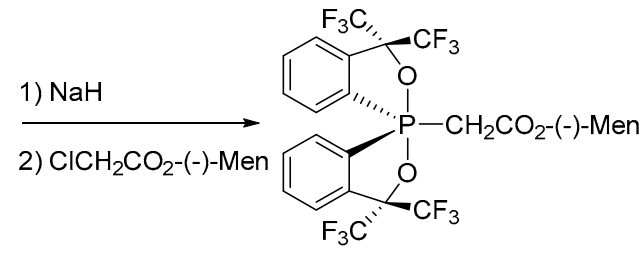

$60-\left(R_{\mathrm{P}}\right), 60-\left(S_{\mathrm{P}}\right)$

$$
\text { mixture of }
$$$$
\text { diastereoisomers }
$$

$1: 1$

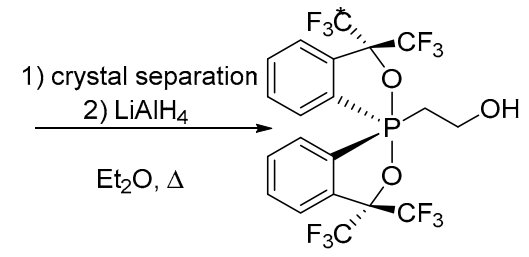

61- $\left(R_{\mathrm{P}}\right)$ and $61-\left(S_{\mathrm{P}}\right)$

Scheme 25. Synthesis of diastereomeric phosphoranes 60 and enantiomeric phosphoranes 61.

The removal of the menthyl unit by treating the diastereoisomers $60-\left(R_{\mathrm{P}}\right)$ and $60-\left(S_{\mathrm{P}}\right)$ with an excess of $\mathrm{LiAlH}_{4}$ provided the enantiomerically pure phosphoranes 61- $\left(R_{\mathrm{P}}\right)$ and $61-\left(S_{\mathrm{P}}\right)$, respectively, with a single chirality center at the pentacoordinated phosphorus atom. In order to verify enantiomeric purity of the obtained $P$-chiral 2-hydroxyethylphosphoranes 61, they were converted into the $(R)-(+)$ Mosher esters 62 using $(R)-(+)-2$-methoxy-2-(trifluoromethyl)phenylacetic acid chloride ((+)-MTPA-Cl)) (Scheme 26) [78].

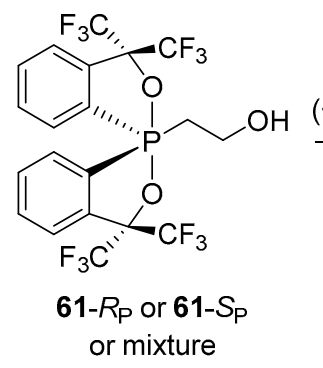

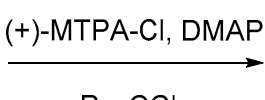

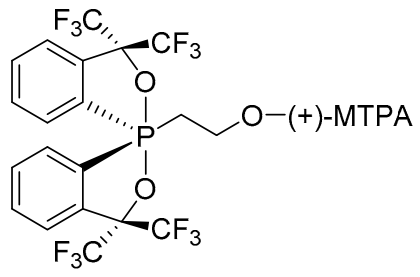

62- $R_{\mathrm{P}}$ or $62-S_{\mathrm{P}}$ or mixture

Scheme 26. Conversion of phosphoranes $\mathbf{6 1}$ to esters 62 .

Independently, the H-phosphorane 59 was converted (by the treatment with $n$-BuLi) into an anion, which was reacted with $\mathrm{CH}_{2} \mathrm{I}_{2}$ affording the alkylating product 63 in $82 \%$ yield. Subsequently $\alpha$-iodomethyl phosphorane derivative was treated with $\alpha$-methylbenzylamine to give a diastereomeric mixture of 64 in $56 \%$ yield (Scheme 27). The pure diastereoisomers were isolated by recrystallization and their absolute configuration was determined by X-ray analysis.

It was also found that deprotonation of the nitrogen-bonded proton in each single diastereomer 64 afforded enantiomeric phosphoroanidates $65-\left(R_{\mathrm{P}}\right)$ and $65-\left(S_{\mathrm{P}}\right)$ (via imine elimination). They, after acidification, gave the desired optically active $\mathrm{P}-\mathrm{H}$ phosphoranes $59-\left(R_{\mathrm{P}}\right)$ and $59-\left(S_{\mathrm{P}}\right)$ (Scheme 28). 


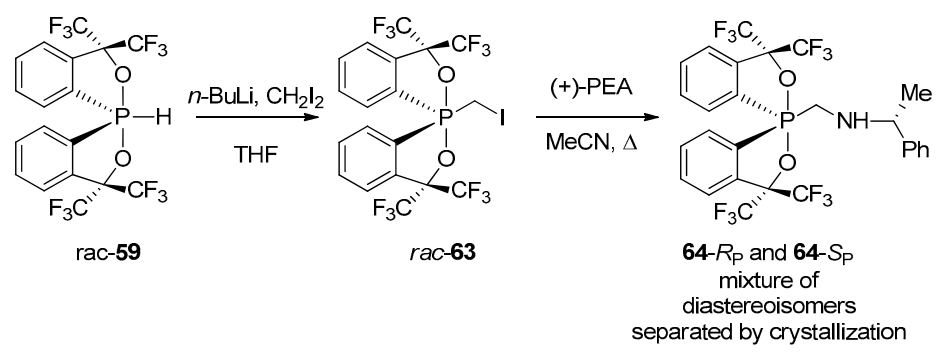

Scheme 27. Synthesis of diastereomeric phosphoranes 64 .

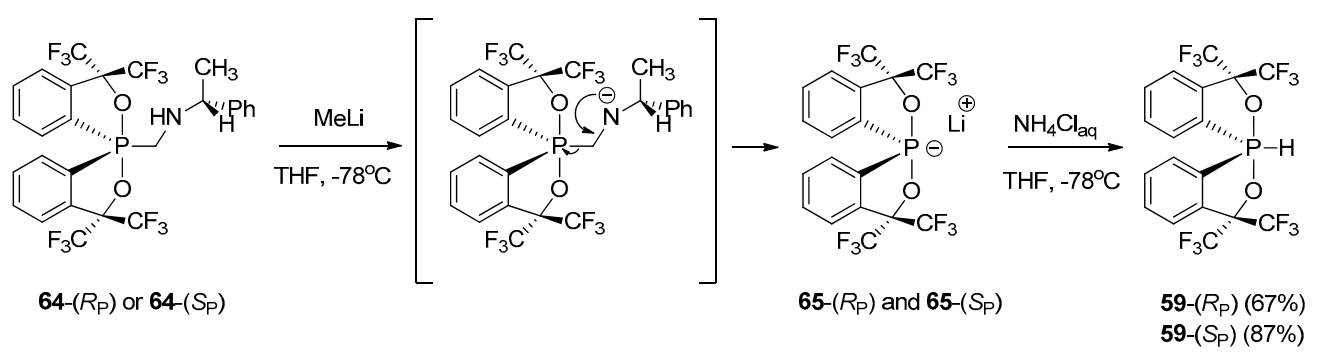

Scheme 28. Conversion of diastereomeric phosphoranes 64 to enantiomeric phosphoranes 59.

Later on, Akiba and co-workers reported the first example of an "anti-apicophilic" (O-cis) spirophosphoranes 67 [79-81], in which the oxygen atom occupies an equatorial position and the carbon atom is located in an apical position of a five membered ring, which is in contrast to the general concept of apicophilicity. According to the procedure shown in Scheme 29 (O-cis) phosphoranes 66 were obtained. The treatment of $\mathrm{P}-\mathrm{H}$ phosphorane 59 with an excess of alkyl(aryl)lithium resulted in the formation of a dianionic intermediate which upon treatment with $\mathrm{I}_{2}$, as an oxidizing agent, afforded P-I equatorial phosphorane. Its cyclization, initiated by the nucleophilic attack of the alkoxide anion at the phosphorous centre and the simultaneous extrusion of $\mathrm{I}^{-}$produced $O$-cis-alkyl-or aryl spirophosphoranes 66.

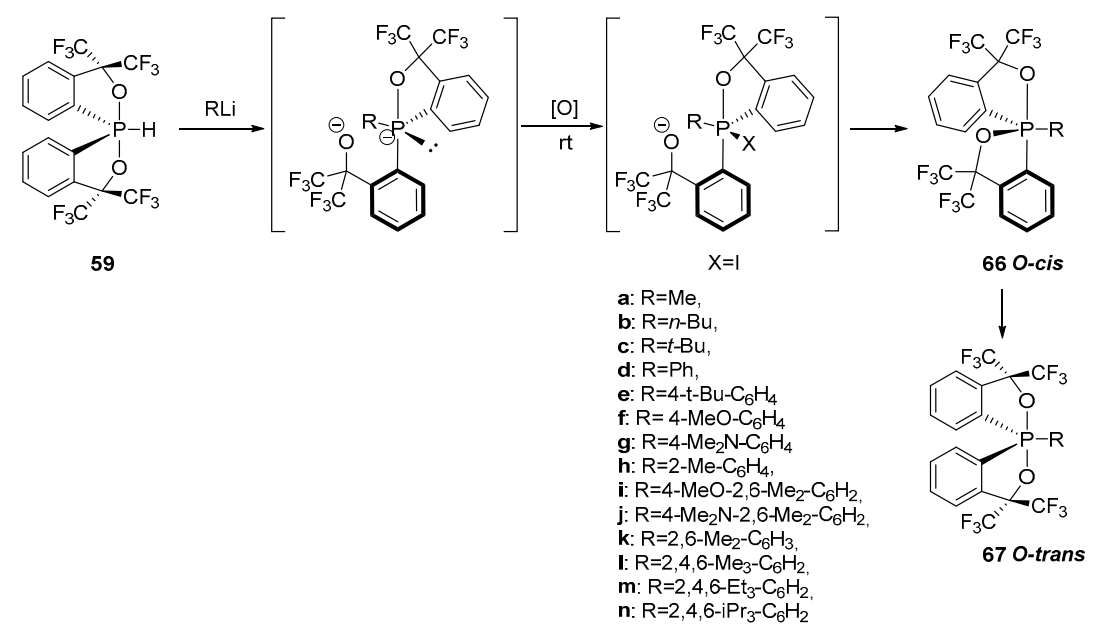

Scheme 29. Synthesis of (O-cis) spirophosphoranes 66 and their stereomutation to (O-trans) phosphoranes 67.

The effects of substituents in the monodentate aryl ring of O-cis arylphosphoranes on the stereomutation process, along with a kinetic study on the stereomutation of O-cis $66 \mathbf{k}-\mathbf{n}$ to O-trans 67k-n were studied in details. The exclusive generation of $O$-cis 66 could be observed for the alkyl derivatives $66 \mathrm{a}-\mathrm{c}$ right after oxidation, however, in the case of the phenyl derivative $66 \mathrm{~d}$ the 
O-cis isomer was the minor product. The pseudorotation to O-trans $67 \mathbf{d}$ isomer was complete in $30 \mathrm{~min}$ at room temperature, which could be explained by the higher apicophilicity of aryl groups in comparison to alkyl groups. For compounds $66 \mathbf{e}-\mathbf{g}$, bearing electron donating groups $(t \mathrm{Bu}, \mathrm{OMe}$, $\mathrm{NMe}_{2}$, respectively) in the 4-position of the monodentate aryl groups $\mathrm{O}$-cis isomers were the major products. Finally, the use of bulky aryl organometallic reagents containing two substituents in ortho positions led to the exclusive formation of the $\mathrm{O}$-cis isomers $(\mathbf{6 6} \mathbf{i}-\mathbf{n})$, having the sufficient lifetimes for characterization. From the relative stability of $6 \mathbf{6 i}-\mathbf{n}$, it is evident that steric effects contributed mainly to the stabilization against pseudorotation in these isomers that exhibited reversed apicophilicity. In the case of $66 \mathrm{n}$, pseudorotation was slow enough to allow for its isolation (purification on silica gel in $71 \%$ yield) and the crystal structure determination by X-ray diffraction. The differences in the reactivity of $O$-cis-66b and $O$-trans-67b toward nucleophiles were also indicated (Scheme 30). The reaction of $O$-cis-66 with TBAF as a nucleophile afforded the hexacoordinated phosphate 68 with the newly formed P-F bond while the trans-isomer did not react. Rapid decomposition of 68 caused by the presence of traces of water was observed. O-Cis $66 \mathbf{b}$ likewise was reacted with MeLi to give product 69, while $O$-trans-67b treated with organolithium reagent remained unreacted under the same conditions; however by increasing MeLi molar equivalency and temperature the product 69 was successfully isolated.

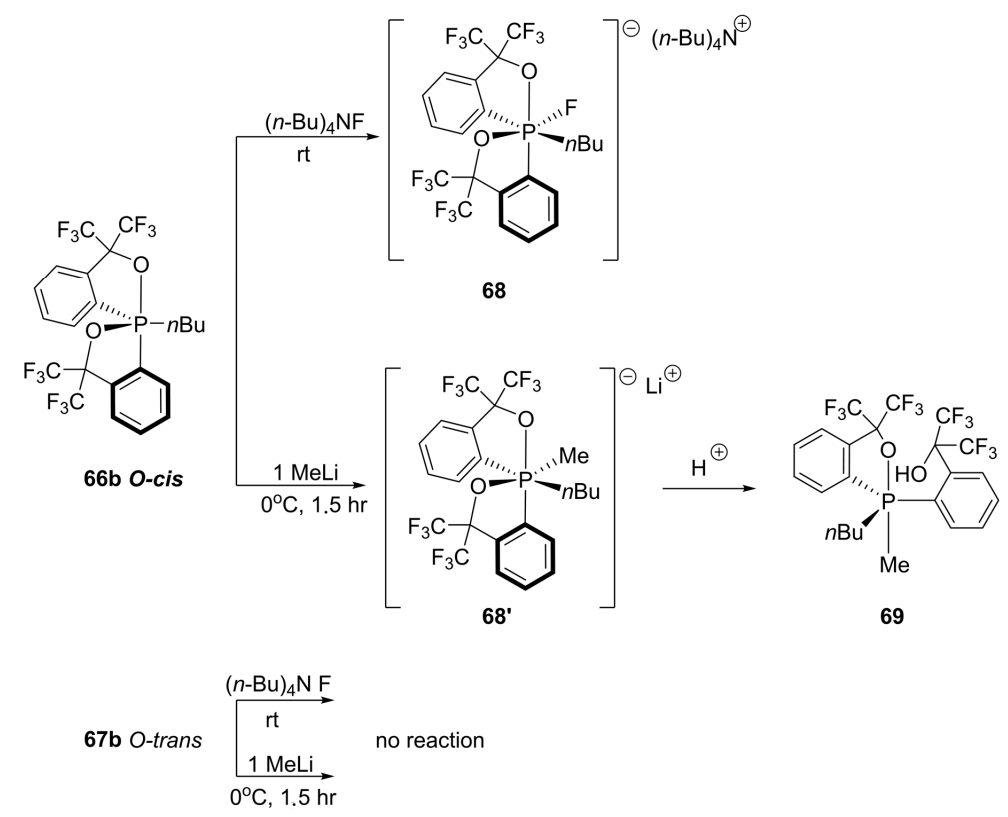

Scheme 30. Reactivity of $O$-cis-66b and $O$-trans-67b toward nucleophiles.

A three-component reaction of phosphoranide 70, silane 71, and THF was applied for the preparation of hypervalent compound $\mathbf{7 2}$ containing simultaneously pentacoordinated phosphorus and silicon atoms (Scheme 31) [82,83]. The ${ }^{31} \mathrm{P}-\mathrm{NMR}$ spectrum indicated the presence of two diastereomeric products in a ratio of $1: 1$.<smiles></smiles>

70
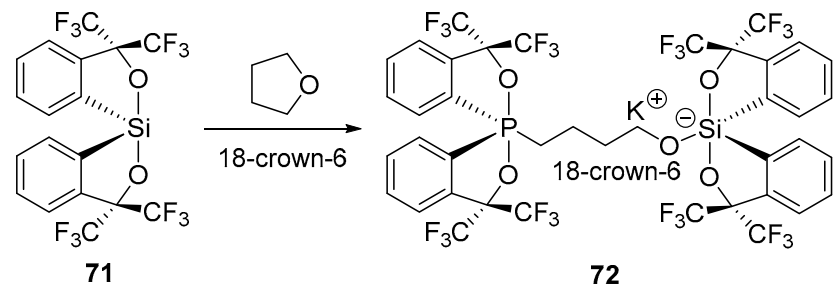

Scheme 31. Reaction of phosphoranide 70 with silane 71. 
The synthesis of other phosphoranylalkoxysilicates was achieved by the nucleophilic addition of phosphoranylalkoxides generated from hydroxy(alkyl)phosphoranes 61 and 73a-c to silane 74 (Scheme 32). Hydroxy(alkyl)phosphoranes 61 and 73a-c were prepared by deprotonation of hydrophosphorane 59 with DBU and subsequent treatment with an electrophile, such as hydrogen peroxide, formaldehyde, 2-bromoethanol, and 4-bromobutanol, respectively. Their treatment with $\mathrm{KH}$ in the presence of 18-crown-6 followed by the addition of silane $\mathbf{7 1}$ in THF afforded the appropriate phosphoranyloxysilicate $74 \mathbf{a}(56 \%)$, and phosphoranylalkoxysilicates $74 \mathbf{b}, \mathbf{c}$ and 72 (53\%-96\%). Potassium silicates $74 \mathbf{a}-\mathbf{c}$ were obtained as a mixture of two diastereomers (in ratios of $66: 34$ for $\mathbf{7 4 a}, 53: 47$ for $\mathbf{7 4 b}, 50: 50$ for $\mathbf{7 4 c}$, respectively), as estimated by the integrals in the ${ }^{31} \mathrm{P}-\mathrm{NMR}$ spectra $[82,83]$.<smiles>FC(F)(F)c1ccccc1[PH]1(c2ccccc2)OC(C(F)(F)F)(C(F)(F)F)O1</smiles>
2) reagent a: $\mathrm{H}_{2} \mathrm{O}_{2}$ b: $\mathrm{CH}_{2} \mathrm{O}$ c: $\mathrm{Br}\left(\mathrm{CH}_{2}\right)_{2} \mathrm{OH}$

59<smiles>OP(O)(OC(c1ccccc1)(c1ccccc1)C(F)(F)F)(c1ccccc1)C(F)(F)F</smiles>

$$
\begin{aligned}
& \text { 73a-d } \\
& \text { a: } n=0 \\
& \text { b: } n=1 \\
& \text { c: } n=4 \\
& \text { 61: } n=2
\end{aligned}
$$

1) $\mathrm{KH}, 18-$ crown-6 2) 64

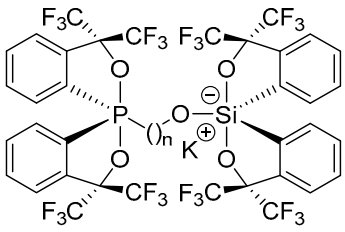

Scheme 32. Reactivity of phosphoranes 59,61 and 73.

More recently, studies on spirophosphoranes, prepared from a new bulkier analogue of the Martin ligand containing two decafluoroetyl units, have been carried out by the Yamamoto group. This new ligand was expected to suppress BPR more efficiently than the Martin ligand [84]. The synthesis of $\mathrm{P}-\mathrm{H}$-spirophosphoranes $\mathbf{7 5 a}, \mathbf{b}$ was based on a two-step reaction involving dimetallation of perfluorocumyl alcohol derivatives leading to the corresponding dianion and its subsequent condensation with $\mathrm{PCl}_{3}$, followed by acidification with 6 molar $\mathrm{HCl}$. The $\mathrm{O}$-equatorial spirophosphoranes $\mathbf{7 6} \mathbf{a}, \mathbf{b}$ were obtained by the reaction of $\mathbf{7 5 a}, \mathbf{b}$ with 3 molar equivalents of RLi followed by the oxidation with $\mathrm{I}_{2}$. Their conversion to the corresponding $O$-apical stereoisomers $\mathbf{7 0 a}, \mathbf{b}$ proceeded in the solution at high temperatures. The stereomutation of 76a-1 to 77a-1 was found to proceed with first-order kinetics. The free energy of activation for stereomutation was higher by $3.6 \mathrm{kcal} / \mathrm{mol}$ in comparison with the $\mathrm{CF}_{3}$ analogue indicating that steric hindrance of pentafluoroethyl group is the major factor for freezing pseudorotation. Recently, Jiang and coworkers [85] obtained a new stable anti-apicophilic phosphorene $\mathbf{7 6} \mathbf{b}$ with four bulky $n-\mathrm{C}_{3} \mathrm{~F}_{7}$ groups, which was converted to more stable $O$-apical phosphorane $\mathbf{7 7 b}$ only by heating, (Scheme 33 ).

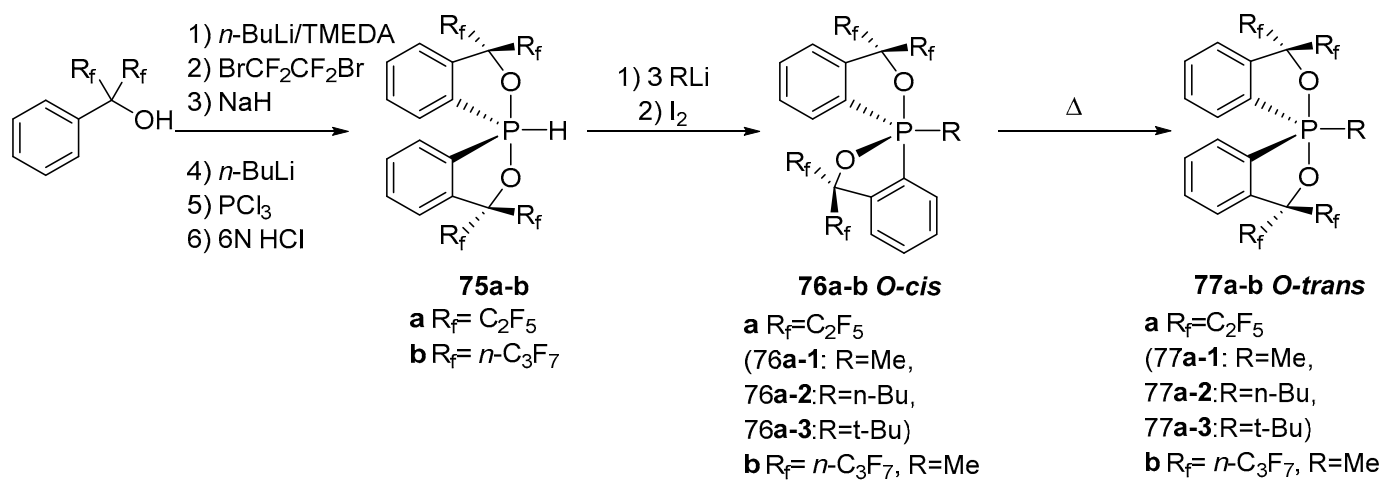

Scheme 33. Synthesis of phosphoranes 76 and 77. 
The same research group elaborated the synthesis of a series of anti-apicophilic phosphoranes bearing a para-substituted aryl group $\left(-\mathrm{C}_{6} \mathrm{H}_{4}(p-\mathrm{X}) ; \mathrm{X}=\mathrm{H}, \mathrm{CF}_{3}, \mathrm{~F}, \mathrm{OMe}\right)$ or a mesityl group as a more electronegative monodentate ligand than an alkyl group. The $O$-equatorial arylphosphoranes $69 \mathbf{c}-\mathbf{g}$ were successfully synthesized by the reaction of the P-H spirophosphorane 68a with an excess of ArLi, followed by treatment with $\mathrm{I}_{2}$ (Scheme 34) [86]. These phosphoranes were found to be stable at r.t. Their isolation indicates that the steric effect of the $\mathrm{C}_{2} \mathrm{~F}_{5}$ groups for freezing the isomerization is remarkable. By heating in an organic solvent the $O$-equatorial arylphosphoranes $69 \mathrm{c}-\mathrm{g}$ were quantitatively converted into the more stable $O$-apical isomers $\mathbf{7 0} \mathbf{c}-\mathbf{g}$. The effect of a solvent and a para-substituent on the rate of the isomerization were also investigated in order to provide an insight into the $\pi \rightarrow \sigma^{*} \mathrm{P}-\mathrm{O}$ interaction in the O-equatorial arylphosphoranes. The kinetic study showed a small para-substituent effect on the stereomutations. However, the multi-regression analysis for the para substituents revealed the 1.3 times greater contribution of the resonance effect on the on the isomerization rate in $\mathrm{C}_{6} \mathrm{D}_{6}$ than the inductive effect suggesting that the $\pi \rightarrow \sigma^{*} \mathrm{P}-\mathrm{O}$ stabilizing interaction in the $\mathrm{O}$-equatorial isomer plays some role in the isomerization.

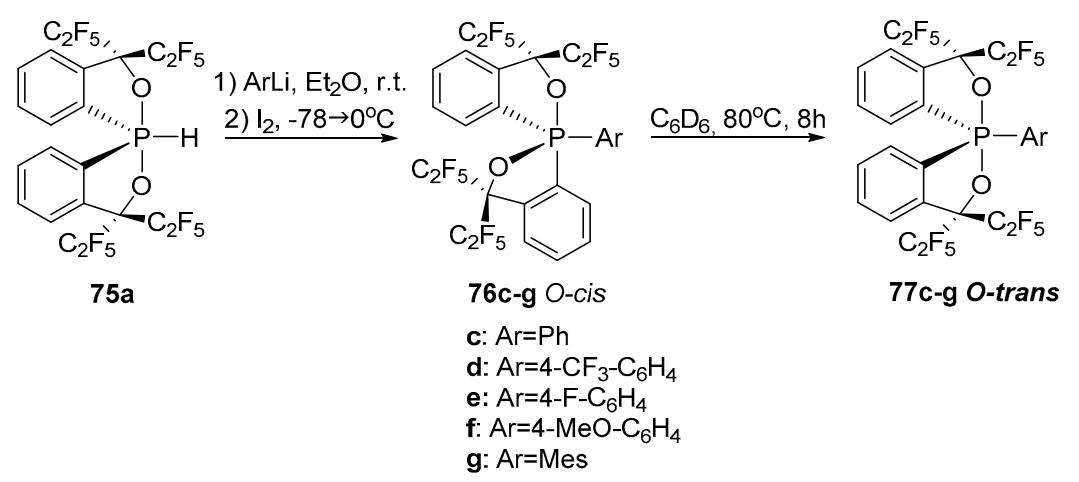

Scheme 34. Synthesis of phosphoranes 76 and their interconversion into 77.

In this context it is interesting to note that the O-equatorial methylphosphorane 76a treated with MeLi was converted to the more stable $O$-apical isomer 77a, but on the contrary, the $O$-apical phosphorane did not react with MeLi at all (Scheme 35) [87].

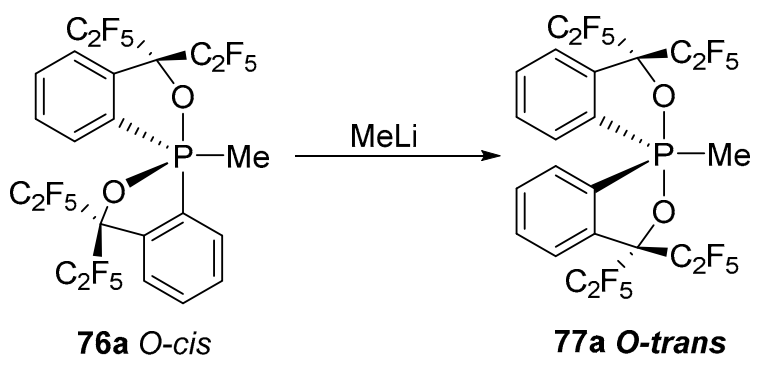

Scheme 35. Methyllithium induced epimerization of $O$-equatorial methylphosphorane 76a.

Deprotonation of methylphosphorane 76a at the methyl group using superbase $(t-\mathrm{BuOK} / n-\mathrm{BuLi})$ led to the corresponding $\alpha$-anion, which upon treatment with electrophilic agents gave new phosphorane derivatives $\mathbf{7 8}$ and/or $\mathbf{7 9}$ (Scheme 36). The $O$-equatorial and $O$-apical phosphoranes having a $\beta$-hydroxyethyl group were synthesized according to this procedure using paraformaldehyde and applied as monodentate ligands [87].

Akiba et al. reported the preparation and characterization of an anti-apicophilic spirophosphorane bearing an oxaphosphetane ring and the Martin ligand [88]. Although the substitution pattern on the phosphorus atom slightly differs from that of oxaphosphetane in the typical Wittig reaction they can be considered as a model for the possible reactive intermediate for the Wittig reaction. 


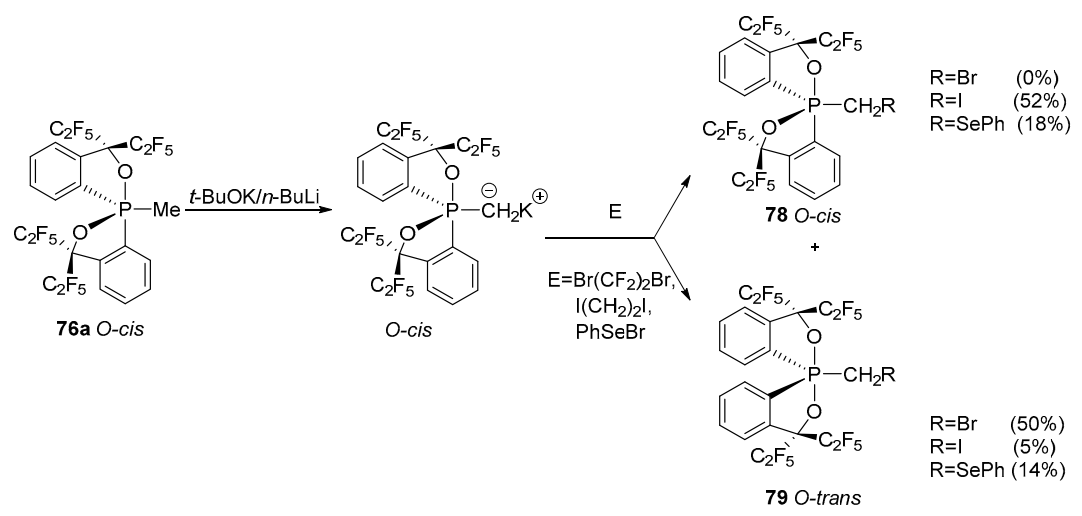

Scheme 36. Synthesis of phosphorane derivatives 78 and/or 79.

The synthesis of isomeric mixture of oxaphosphetane $\mathbf{8 0 a}$ and $\mathbf{8 0 b}$ in 1:1 ratio was achieved by the oxidation procedure using $n$-BuLi followed by iodine in ether (Scheme 37). The sole anti-apicophilic 80a isomer was isolated from the reaction mixture by crystallization upon addition of hexane. Under acidic conditions 80a underwent complete stereomutation to 80b. A similar conversion took place within minutes upon dissolution of $\mathbf{8 0 a}$ in anhydrous $\mathrm{CDCl}_{3}$ at room temperature. A ${ }^{31} \mathrm{P}-\mathrm{NMR}$ measurement of the $80 \mathrm{~b}$ sample heated to $120^{\circ} \mathrm{C}$ in $p$-xylene did not show the presence of $80 \mathrm{a}$ in equilibrium, indicating that $\mathbf{8 0 a}$ is thermodynamically much less favorable than its $O$-apical isomer. Moreover, heating a solid sample of $\mathbf{8 0 a}$ at $120^{\circ} \mathrm{C}$ for $5 \mathrm{~min}$. gave only $\mathbf{8 0 b}$ as a result of pseudorotation.

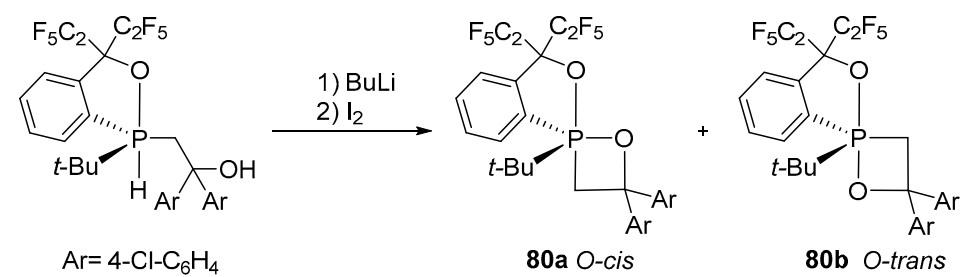

Scheme 37. Synthesis of isomeric mixture of oxaphosphetane $80 \mathrm{a}$ and $80 \mathrm{~b}$.

The synthesis of pentacoordinate phosphirenes 82a and $82 \mathbf{b}$ bearing a phenyl group on the phosphorus atom was achieved by the reaction of tricoordinate phosphirenes 81a and 81b with o-chloranil (Scheme 38) [89].

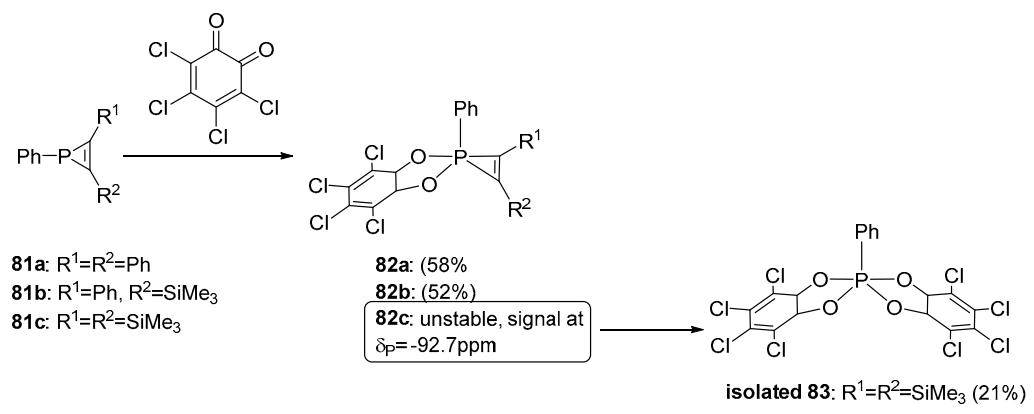

Scheme 38. Reaction of phosphirenes $81 \mathrm{a}$ and $81 \mathrm{~b}$ with $o$-chloranil.

The formation of 82c was evidenced by ${ }^{31} \mathrm{P}-\mathrm{NMR}$ spectrum that showed a suitable signal at $\delta_{P}-92.7$. It was converted rapidly to phosphorane 83 bearing two tetrachlorocatecholate ligands. For relatively more thermally stable phosphirene 82a the crystal structure was determined by X-ray analysis. It showed that phosphirene 82a has a highly distorted square pyramidal SP arrangement 
at the phosphorus atom as a core. The basal positions are occupied by two oxygen atoms from the tetrachlorocatecholate ligand and two carbon atoms belonging to the three-membered ring of phosphirene, when the phenyl group is located at the apical position. No decomposition was observed for 82a after heating at $60{ }^{\circ} \mathrm{C}$ for $40 \mathrm{~h}$. On the other hand, phosphirene 82b was thermally less stable, and decomposed at room temperature to give the $o$-chloranil derived phenylphosphinite and phenyl(trimethylsilyl)acetylene. These results indicate that an attachment of a trimethylsilyl group to the endocyclic carbon lowers the thermal stability of the pentacoordinate phosphirenes.

\section{P-3C-1O-1Se; 10P-3C-1O-1S; 10P-3C-1O-1F; 10P-3C-1O-1H Phosphoranes}

The synthesis of the first $1,2-\sigma^{5-}$ selenaphosphirane and $1,2-\sigma^{5}$-thiaphosphirane involving a pentacoordinate phosphorus atom was based on the reaction of the Martin ligand based phosphorus ylide with elemental selenium [90], CpTiSe ${ }_{5}$ [91] or elemental sulfur [92]. Treatment of ylide 84 with 1.6 equiv. of elemental selenium in THF at room temperature or with $\mathrm{Cp}_{2} \mathrm{TiSe}_{5}$ under the same conditions resulted in the formation of selenaphosphirane 85 as a solid in $96 \%$ and $85 \%$ yield, respectively (Scheme 37). Although the selenaphosphirane $\mathbf{8 5}$ is highly moisture-sensitive, its isolation was successful under an argon atmosphere and its structure was crystallographically characterized. Both, the solid-state ${ }^{31} \mathrm{P}\left\{{ }^{1} \mathrm{H}\right\}$ NMR spectrum and the ${ }^{31} \mathrm{P}\left\{{ }^{1} \mathrm{H}\right\}$ NMR spectrum of the $\mathrm{C}_{6} \mathrm{D}_{6}$ solution showed a single peak with chemical shifts at -26.1 and -26.6 , respectively. This was considered as evidence for the pentacoordinate state of the phosphorus atom of the selenaphosphirane. It is interesting to note that in the ${ }^{77} \mathrm{Se}\left\{{ }^{1} \mathrm{H}\right\}-\mathrm{NMR}$ spectrum (the resonance signal at $\delta_{\mathrm{Se}} 147.5$ in $\mathrm{C}_{6} \mathrm{D}_{6}$ ), the coupling constant between the phosphorus and the selenium nuclei was not observed. This selenaphosphirane upon treatment with methyl triflate in $\mathrm{CDCl}_{3}$ afforded the highly air-sensitive $\alpha$-(methylseleno)- $\alpha$-methylethyl phosphonium triflate 86 in $76 \%$ yield (Scheme 39). The formation of 86 was initiated by the electrophilic attack of methyl triflate on the negatively charged selenium atom in 85 and proceeded with the subsequent cleavage of the polarized P-Se bond. In a similar manner the thiaphosphirane 87 was prepared in $68 \%$ yield, by the reaction of the ylide 84 with 0.96 equivalent of elemental sulfur in THF at $-30{ }^{\circ} \mathrm{C}$ for $7 \mathrm{~h}$ (Scheme 39). Thiaphosphirane $\mathbf{8 7}$ was found to be air-sensitive and its single crystal was obtained by recrystallization from hexane under an argon atmosphere. Its X-ray crystallographic analysis showed that the oxygen and sulfur atom are located at apical positions.

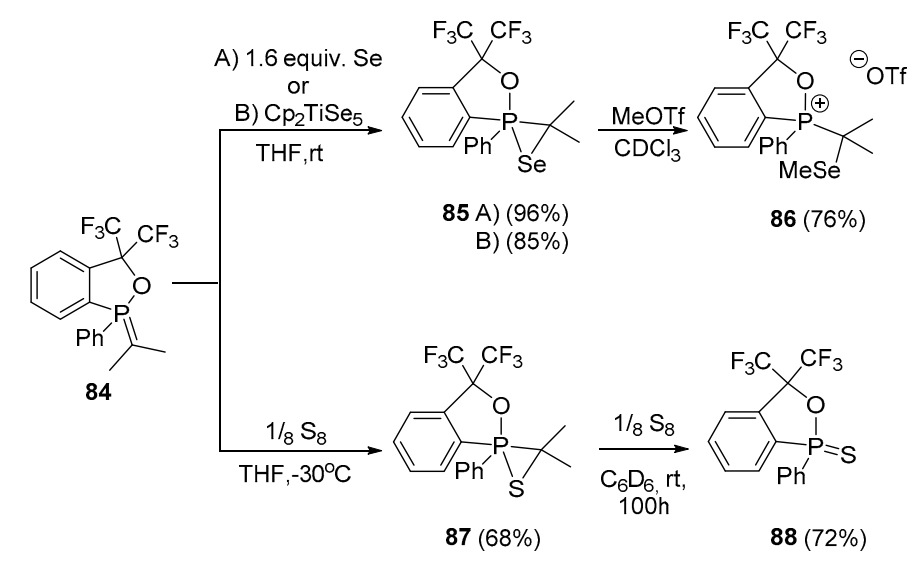

Scheme 39. Reactions of monocyclic phosphorus ylide 84 .

Thiaphosphirane $\mathbf{8 7}$ showed inertness toward triaryl- or trialkylphosphines. This is in a contrast to tri- and tetracoordinate thiaphosphiranes which underwent desulfurization to form phosphaalkene derivarives and phosphine sulfides [93]. When the thiaphosphirane 87 was treated with additional portion of elemental sulfur the corresponding phosphinothionate 88 was isolated in $72 \%$ yield. The independent experiment showed that the phosphorus ylide 84 treated with 2 equiv. of elemental sulfur provided phosphinothionate 88 in $31 \%$ yield. 


\section{P-2C-2N-1O; 10P-2C-1N-2O Phosphoranes}

The reactions of iminophosphorane bearing the Martin ligand and a bulky 2,4,6-triisopropyl-phenyl group with a ketone, an isothiocyanate and an alkyne has been found to proceed with the formation of the corresponding cycloadducts as novel heterocycles bearing pentacoordinated phosphorus atom [94,95]. Cyclic iminophosphinate 89 reacted with carbonyl compounds, phenyl isothiocyanate and phenylethynyl trifluoromethyl sulfone affording the appropriate cycloadducts: 1,3,2 $\lambda^{5}$-oxazaphosphetidines 90a-c, 1,3,2 $\lambda^{5}$-diazaphosphetidine-4-thione 91, 1,2 $\lambda^{5}$-azaphosphetine 92, respectively. The reaction with hexafluoroacetone proceeded at room temperature yielding cyclic phosphorane which could be even purified by silica gel chromatography. With dimethyl acetylenedicarboxylate (DMAD) and water the reaction provided 1,2 $2 \lambda^{5}$-oxaphosphol-5(2H)-one 93 instead of the expected 1,2-azaphosphetine (Scheme 40).

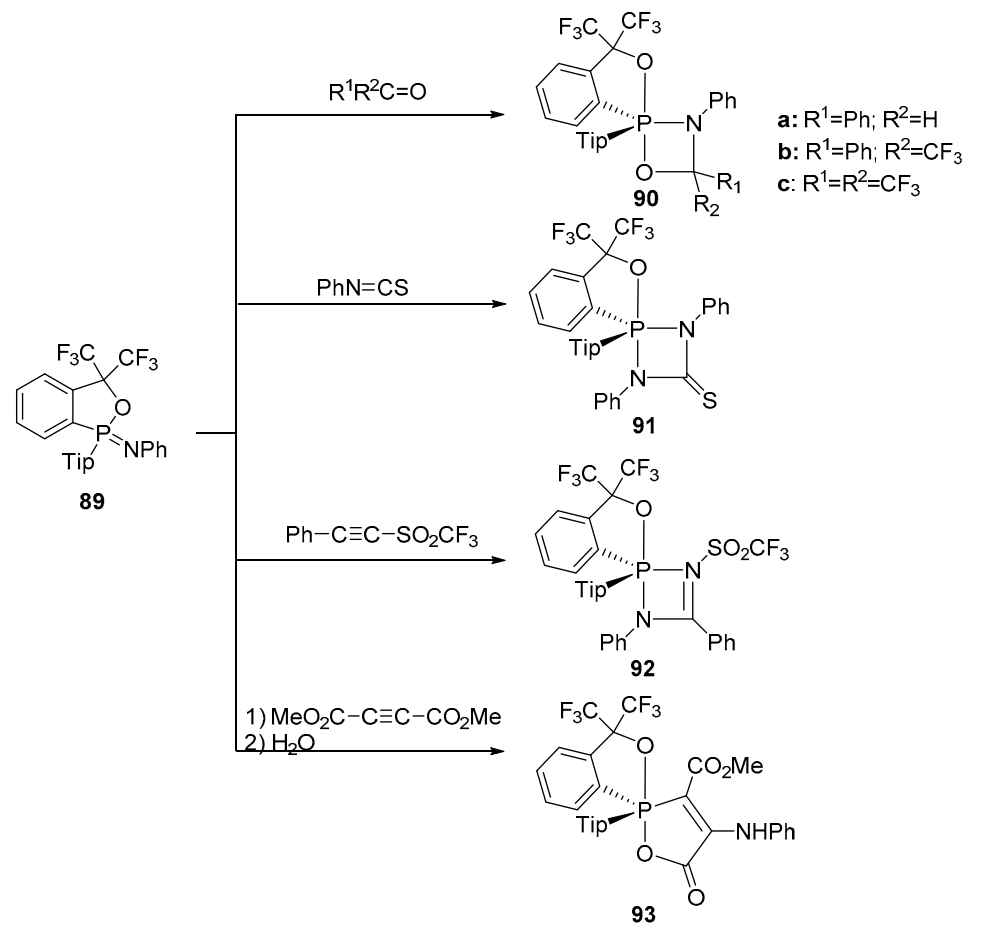

Scheme 40. Syntheses of bicyclic phosphoranes 90-93.

Thermolysis of 90c at $140{ }^{\circ} \mathrm{C}$ in a sealed tube gave the corresponding imine 94 and cyclic phosphinate 95 (Scheme 41), indicating that 1,3,2 $\lambda^{5}$-oxazaphosphetidine $\mathbf{9 0} \mathrm{c}$ is regarded as an intermediate of the aza-Wittig reaction [95].

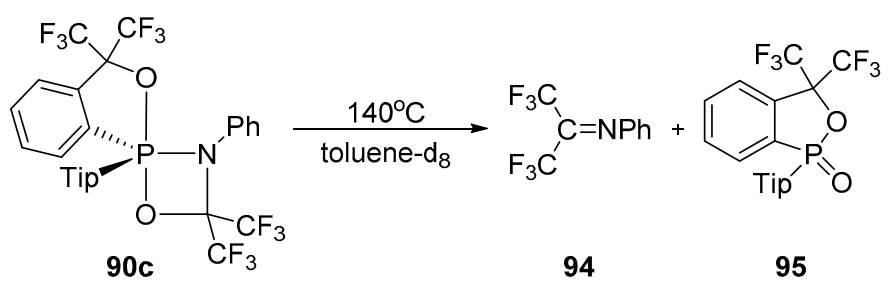

Scheme 41. Thermolysis of spirophosphorane 90c.

The detailed studies on the formation of $1,2 \lambda^{5}$-oxaphosphol-5(2H)-one 93 were conducted on the basis of ${ }^{31} \mathrm{P}-\mathrm{NMR}$ analysis [96]. In the course of the reaction a decrease of the resonance signal at +25.6 ppm along with an increase of the new one at +47.3 ppm corresponding to 96 was observed. 
The latter was found to be unstable in the presence of moisture which resulted in the slow conversion to the final product. The proven synthetic pathway was concluded in Scheme 42.
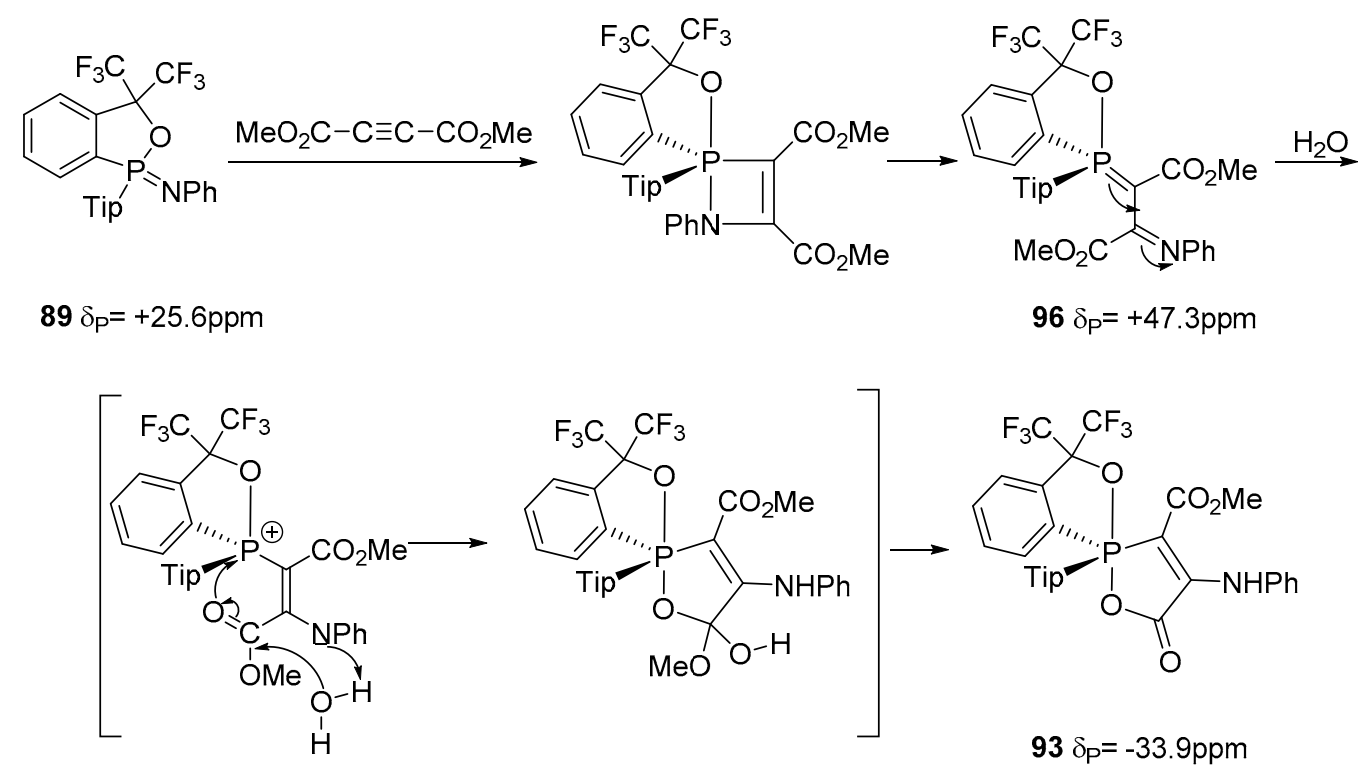

Scheme 42. The mechanism of formation of the bicyclic phosphorane 93 from cyclic iminophosphinate 89.

10P-2C-2O-1Z ( $\mathrm{Z}=\mathrm{N}, \mathrm{O}$, etc.) Phosphoranes

It is already well known that the preparation of pentacoordinate phosphoranes can be accomplished by the reaction of phosphorus (III) compounds with ylidene derivatives of $\beta$-carbonyl compounds. This approach was exemplified by the reaction of 2-phenyl-1,3,2-benzodioxaphosphorin-4-one $\mathbf{9 7}$ with diethyl benzylidenemalonate 98 which occurred along two pathway, yielding pentacoordinate phosphorane $\mathbf{9 9}$ and phosphinate derivative $\mathbf{1 0 0}$ containing tetracoordinate phosphorus atom [97]. When the reaction was allowed to heat for a short time the formation of the seven-membered phosphonate derivative with relatively high stereoselectivity equal to $50 \%$ was observed (Scheme 43 ).

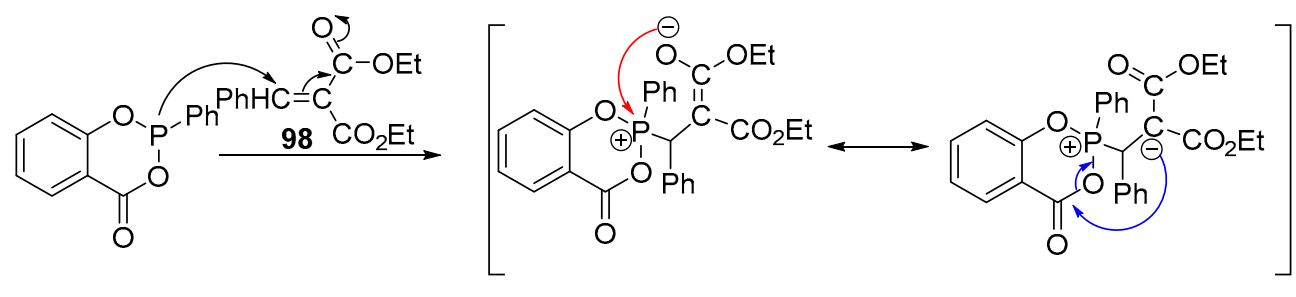

97

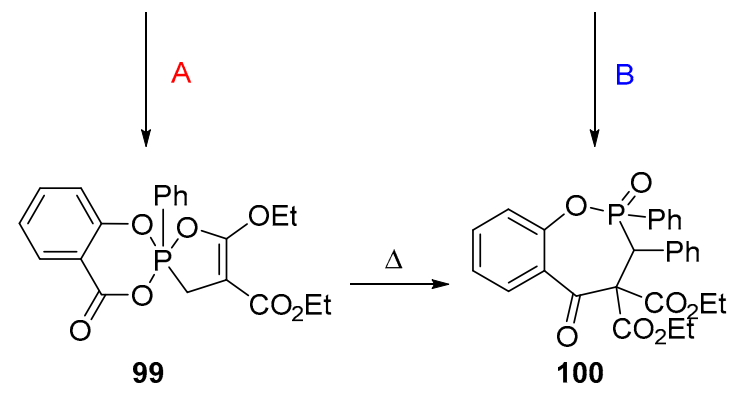

Scheme 43. Synthesis of pentacoordinate phosphorane 99 and phosphinate derivative 100.

The plausible mechanism involves the nucleophilic attack of the phosphorus atom at the $\beta$-carbon atom in $\alpha, \beta$-unsaturated carbonyl compounds to produce an intermediate with delocalized $\pi$-electrons 
along three atoms, as is presented by its two resonance structures. In case of synthetic pathway A the attack of negatively charged oxygen on phosphorus atom occurs with reversible formation of pentacoordinate phosphorane. The second synthetic pathway (B) assumes nucleophilic substitution at the carbonyl carbon atom to yield the seven-membered ring, 1,2-benzoxaphosphepine. In further investigations Akiba et al. [98] succeeded in preparing spirophosphoranes bearing Martin ligand units and a monodentate primary amino substituent. These $O$-equatorial isomers were found to be surprisingly stable, taking into account the fact that the equatorial substituents, which are more electronegative than carbon, are expected to facilitate pseudorotation. The $O$-equatorial aminophosphoranes 102 along with $O$-apical isomers 103 were obtained from the in-situ generated chlorophosphorane 101 which was allowed to react with the corresponding amines (Scheme 44). However, when methylamine or aniline were used, the corresponding $\mathrm{O}$-equatorial isomer were not produced.

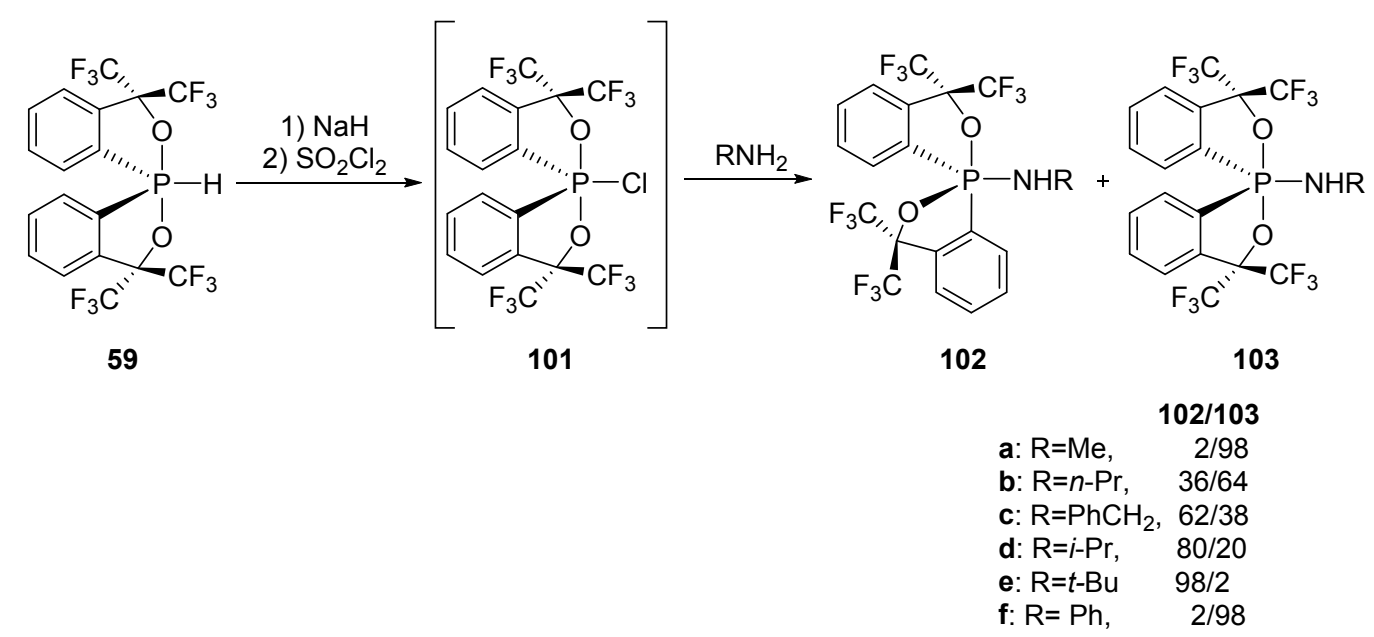

Scheme 44. Synthesis of aminophosphoranes 102 and 103.

The O-equatorial phosphoranes $\mathbf{1 0 2}$ were stable at room temperature and could still be converted to their more stable $O$-apical pseudorotamers 103 when they were heated in a solution, indicating that the phosphoranes $\mathbf{1 0 2}$ are kinetic products, as in the case of $O$-equatorial alkylphosphoranes. The structures of $102 \mathrm{c}$ and 102e were determined by single-crystal X-ray analyses to be in the $O$-equatorial configuration. The unusual stability of the $O$-equatorial phosphorane $\mathbf{1 0 2}$ could be attributed to the stabilizing $\mathrm{n}_{\mathrm{N}} \rightarrow \sigma^{*} \mathrm{P}$-O orbital interaction. More detailed studies on the experimental determination of the $\mathrm{n}_{\mathrm{N}} \rightarrow \sigma^{*} \mathrm{P}$-O interaction energy of $O$-equatorial $C$-apical phosphorane supported with DFT calculations have been also reported providing an additional proof for this assumption.

Juge et al. described the formation of chiral bicyclic phosphoranes in the Arbuzov-type reaction of diastereomerically pure cyclic tricoordinated oxaphosphacycloalkanes and very reactive Koshland reagent I 104 [99]. They found that both enantiomers of 2-phenyl-1.3.2-oxazaphospholidine [(-)-105 derived from (+)-ephedrine or (+)-105 from (-)-ephedrine], treated with 2-hydroxy-5-nitrobenzyl bromide 104a afforded the stable pentacoordinated phosphoranes $[(-)-106$ from $(-)-105$ or (+)-106 from (+)-105] which were isolated by simple chromatography. The minor isomeric spirophosphoranes with the opposite configuration at the phosphorus atom were also formed (Scheme 45). 2-Hydroxy-5-nitrobenzyl halides 104a, b reacted also with five- or six-membered cyclic tricoordinated phosphorus compounds (-)-107 (derived from (+)-2-methyl-2,3-butanediol) or (-)-110 (derived from (-)-chloramphenicol), yielding spirophosphoranes, 108 and 109 or 110, respectively. However, these spirophosphoranes were too unstable to be isolated. Their formation was confirmed only by the ${ }^{31} \mathrm{P}-\mathrm{NMR}$ spectra of a crude reaction product. 


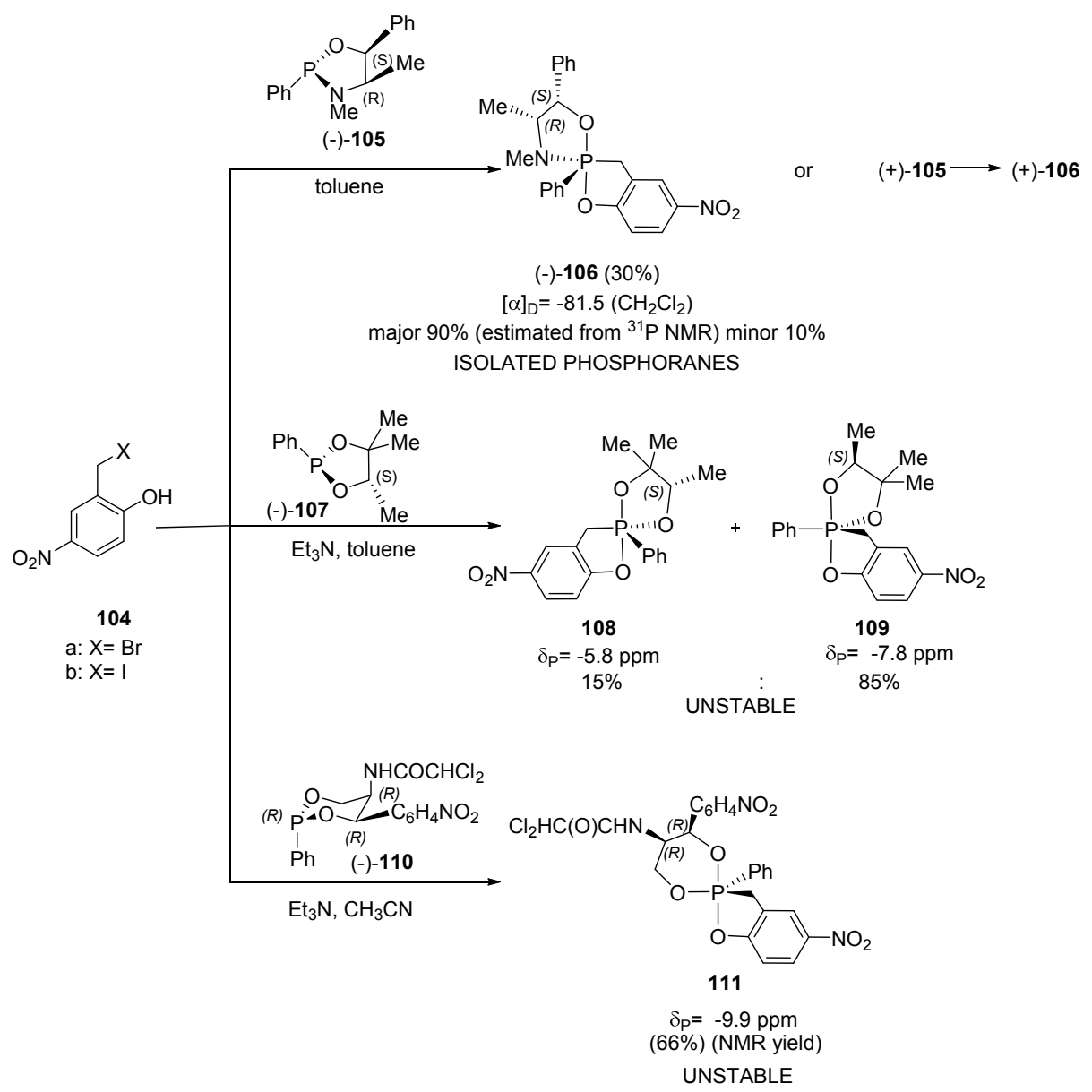

Scheme 45. Reactions of cyclic five or six-membered tricoordinated phosphorus compounds with 2-hydroxy-5-nitrobenzyl halides.

\section{P-1C-1N-3O Phosphoranes}

The synthesis of a pentacoordinated phosphorane containing tricyclic system was reported by Sevenard et al. [100]. 2-Fluoroacetylcycloalkanones 111a,b reacted with diethyl isocyanatophosphite diastereospecifically leading to phosphoranes $\mathbf{1 1 2} \mathbf{a}, \mathbf{b}$ via intermediate species formed by the addition of phosphorus to a trifluoromethyl substituted carbonyl atom which subsequently underwent two additional heterocyclizations (Scheme 46).

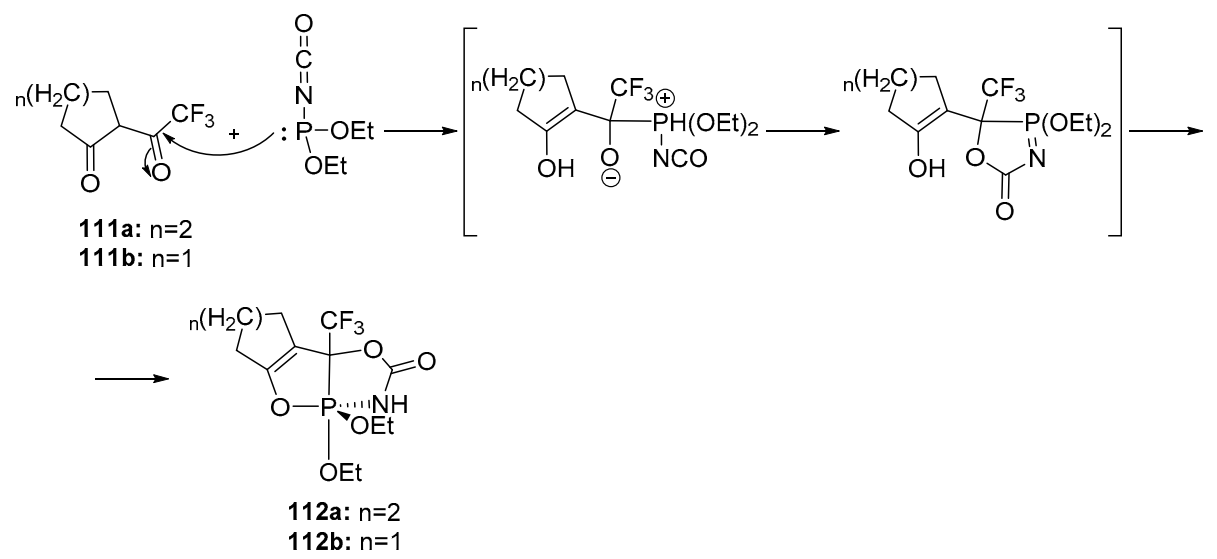

Scheme 46. Synthesis of bicyclic aminophosphoranes 112. 
The phosphorane derived from 2-trifluoroacetylcyclopentanone $\mathbf{1 1 2 b}$ was moisture-sensitive, unlike the cyclohexane derivative 112a, probably due to the greater ring strains. The proposed structure was strictly confirmed by means of NMR data on the basis of which it was found that carbon atom occupies an axial position and two annulated five membered rings are arranged in the equatorial-axial-equatorial order.

\section{P-1C-2N-2O Phosphoranes}

Pudovik et al. reported the synthesis and crystal structure analysis of spirophosphoranes derived from benzoxazaphospholidines and/or 2-aminophenol [101]. It has been found that the condensation reaction of 2-aminophenol with chloromethylphosphonic dichloride proceeded with the formation of the expected benzoxazaphospholidine-2-oxide 99 along with the unexpected one: spirophosphorane 100 (Scheme 47).

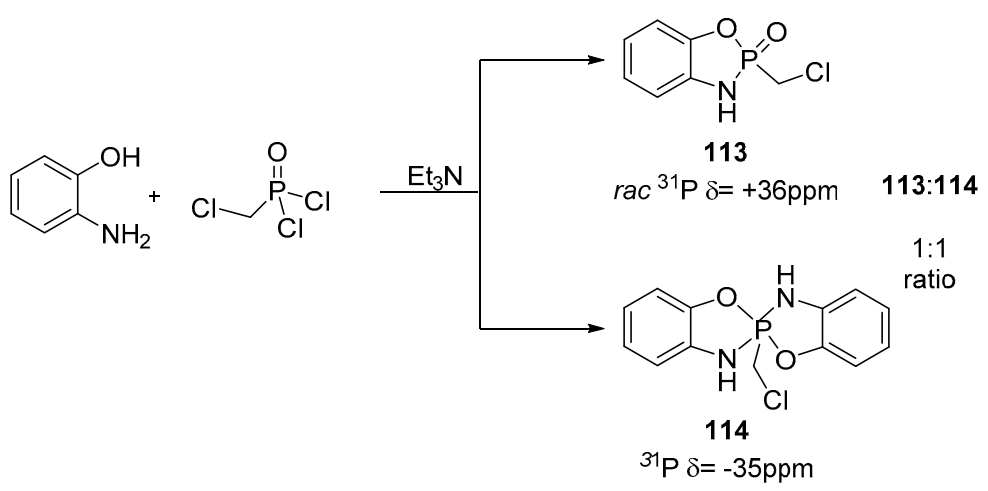

Scheme 47. Synthesis of bicyclic aminophosphorane 114.

As indicated by the authors, the crucial step in a plausible pathway for their synthesis is an initial phosphorylation of a hydroxy group in 2-aminophenol with phosphonic dichloride leading to chloromethylphosphonochloridate intermediate (I). Subsequent nucleophilic attack of the amino group at the phosphorus atom leads to the cyclization product 99. Simultaneously, the second pathway is realized when chloromethylphosphonochloridate intermediate reacts with the next molecule of the starting aminophenol to give the $\mathrm{OH}$ condensation product, which undergoes cyclization to provide unstable intermediate (II, III). It is then converted to the spirophosphorane as a result of intramolecular nucleophilic attack of the amino group at electrophilic phosphorus atom with the elimination of water (Scheme 48).

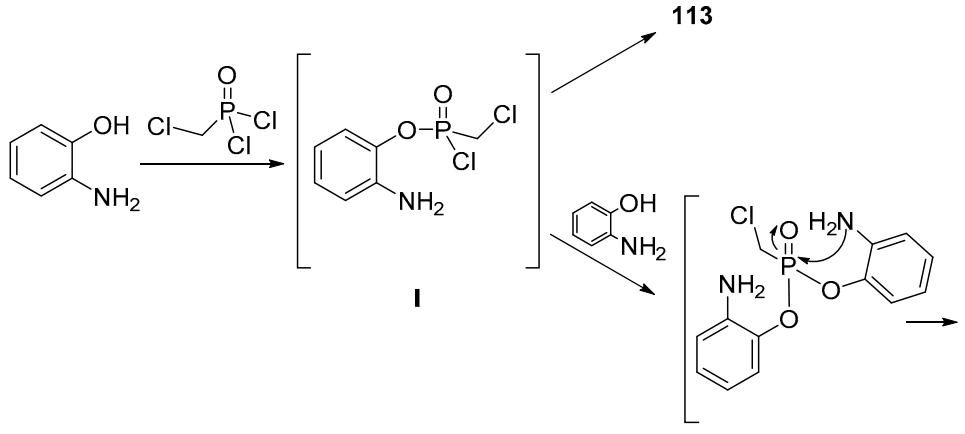

II

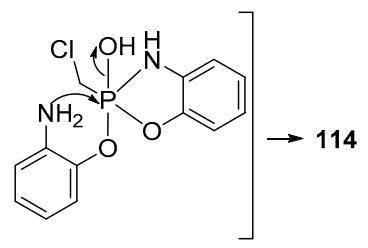

III

Scheme 48. The mechanism for the formation of spirophosphorane 114.

The alkylation of 2-alkoxy-1,3,2-benzoxazaphospholidine with bis(dialkylamino)methanes afforded $\mathrm{N}$-alkylated product $\mathbf{1 1 5}$ which underwent tautomerization to phosphonimidate $\mathbf{1 1 6}$ and 
further transformation to polycyclic phosphoranes 117 in 30 days [102]. It is worth to mention that both benzoxazaphospholidine $\mathbf{1 1 5}$ and polycyclic phosphorane $\mathbf{1 1 7}$ were used as phosphorylating agent for 2-aminophenol yielding spirophosphorane 118 (Scheme 49). The crystal and molecular structure of the synthesized spirophosphoranes $\mathbf{1 1 4}$ and $\mathbf{1 1 8}$ were determined by single-crystal X-ray diffraction $[101,103,104]$.

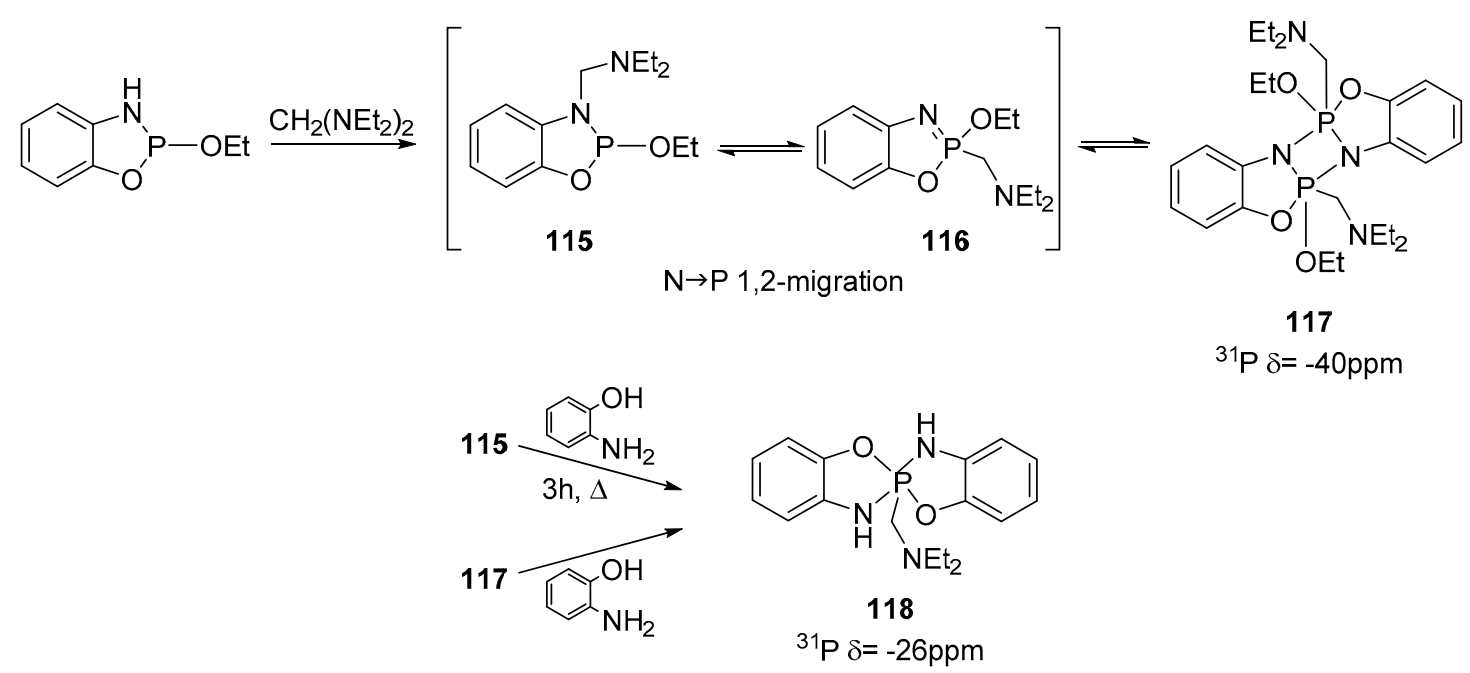

Scheme 49. Syntheses of bicyclic diaminophosphoranes 117 and 118.

Amino alcohol based monocyclic hydrophosphorane 119 was prepared in the reaction of 2-aminophenol with tetraalkylphenylphosphonous diamide and used for the preparation of bicyclic spirophosphorane $\mathbf{1 2 0}$ [105] based on the oxidation procedure or on heating above its melting point (Scheme 50).

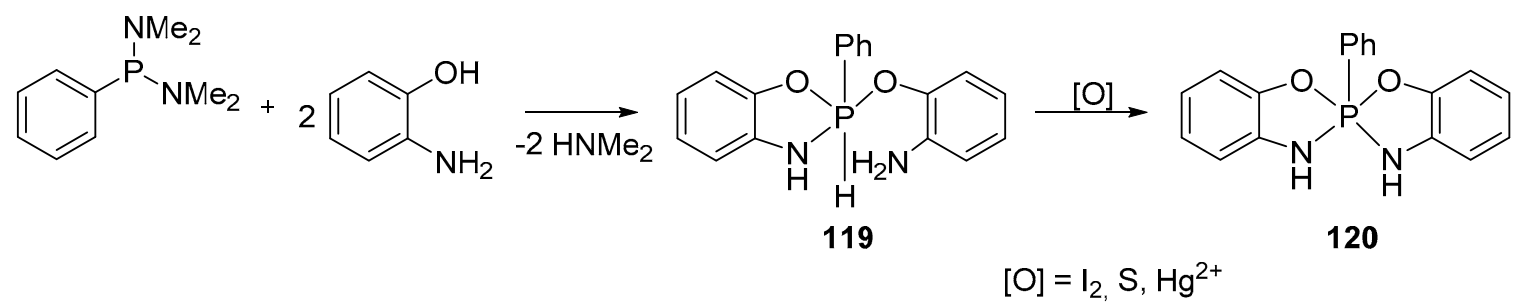

Scheme 50. Synthesis of bicyclic diaminophosphorane 120.

The alternative approach to synthesize bicyclic pentacoordinate spirophosphoranes proposed by Gholivand et al. [106] is based on the condensation of simple hydrazides (i.e., benzhydrazide 123a and 4-pyridinecarboxylic acid hydrazide $\mathbf{1 2 3 b}$ ) with phosphoryl reagents followed by a dehydration-cyclization rearrangement of the resulting phosphorylated hydrazides. Such spiro-bicyclophosphorane with a trigonal bipyramidal structure is formed if phosphoryl reagent contains at least two appropriate leaving groups such as chlorine atoms (in $\mathrm{PhPOCl}_{2}$ or $\mathrm{POCl}_{3}$ ). Benzhydrazide reacted with $\mathrm{POCl}_{3}$ in refluxing acetonitrile to give the $\mathrm{P}-\mathrm{Cl}$ phosphorane intermediate $\mathbf{1 2 1}$ which treated with the appropriate amine was converted to the P-N spirophosphorane 122a, b (Scheme 51). The mechanism proposed for the formation of the intermediate involved $\beta$-amidic proton elimination in phosphorylated hydrazides $\mathbf{A}$ upon which the cyclization product with new $\mathrm{C}=\mathrm{N}$ imine bond $\mathbf{B}$ was formed. The intermediate was finally converted into 121 by dehydration. 
<smiles>NNC(=O)c1ccccc1</smiles>

$123 a$

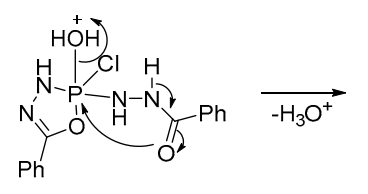

C

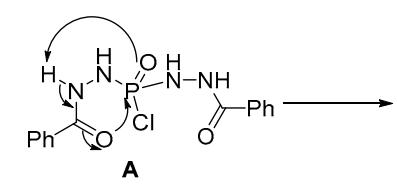

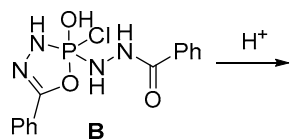

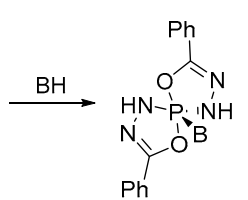

122

Scheme 51. Syntheses of bicyclic diaminophosphoranes 121 and 122.

Similarly, several pentacoordinate phosphoranes $122 \mathrm{c}-\mathrm{g}$ were easily prepared in the reaction of benzhydrazide 123a or 4-pyridinecarboxylic acid hydrazide 123b with $\mathrm{PhPOCl}_{2}, \mathrm{PhNHPOCl}_{2}$ or $\mathrm{POCl}_{3}$ in the presence of triethylamine [106] (Scheme 52).

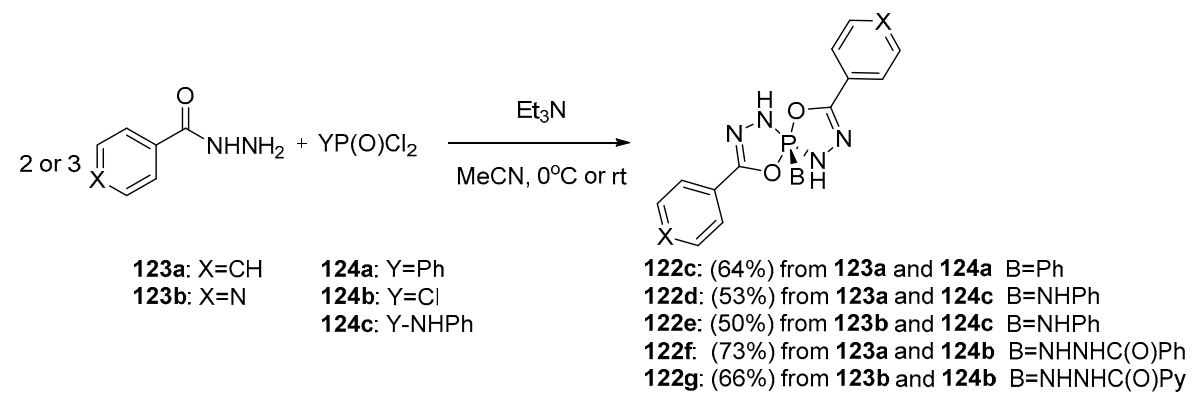

Scheme 52. Synthesis of bicyclic diaminophosphoranes 122.

\section{P-1C-4O Phosphoranes}

The reaction of $(E)$-bis-(2,4,6-tri-t-butylphenyl)diphosphane 125 with a fourfold excess of tetrachloro-o-benzoquinone $\mathbf{1 2 6}$ was found to give the chiral perchlorinated spirophosphorane $\mathbf{1 2 7}$. It was suggested that the reaction, proceeding according to an electron transfer mechanism, occurred via 128 with a cleavage of the P-P bond and indeed, 127 was isolated in low yield even with two equivalents of $\mathbf{1 2 6}$ (Scheme 53) [107].

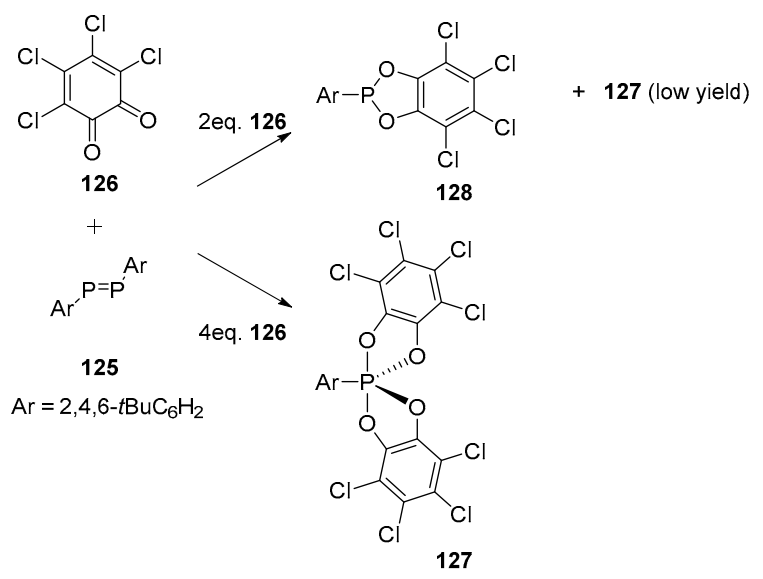

Scheme 53. Synthesis of bicyclic phosphoranes 127. 
Similarly, the reactions of tricoordinated organophosphorus precursors 129-131 with an appropriate equivalent of ethylene glycol was found to give the spirophosphorane 132. All the three reactions occurred via the bis-ester 133 formed in a nucleophilic exchange reaction at the tricoordinated phosphorus center of precursors 129-131 [108] (Scheme 54).

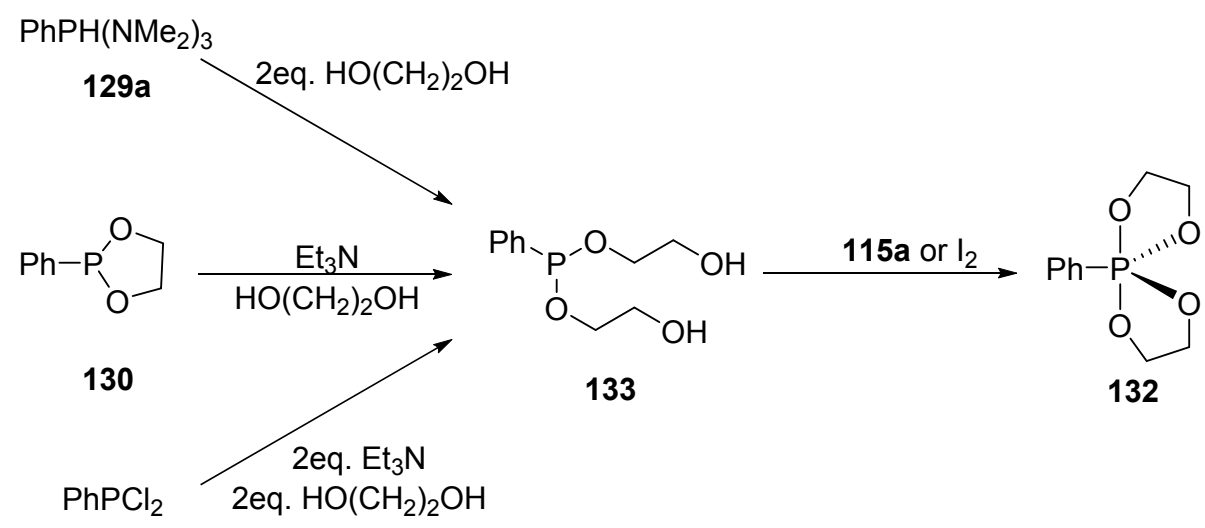

131

Scheme 54. Synthesis of bicyclic phosphorane 132.

\section{P-1C-1N-2O-1H Phosphoranes}

The reactions of tricoordinated phosphonous diamides $\mathbf{1 1 5} \mathbf{a}-\mathbf{d}$ with an appropriate equivalent of aminodiols $\mathbf{1 2 0}$ led to a series of chiral enantiomeric or diastereomeric bicyclic spirophosphoranes 121a-f (Scheme 55) [109].

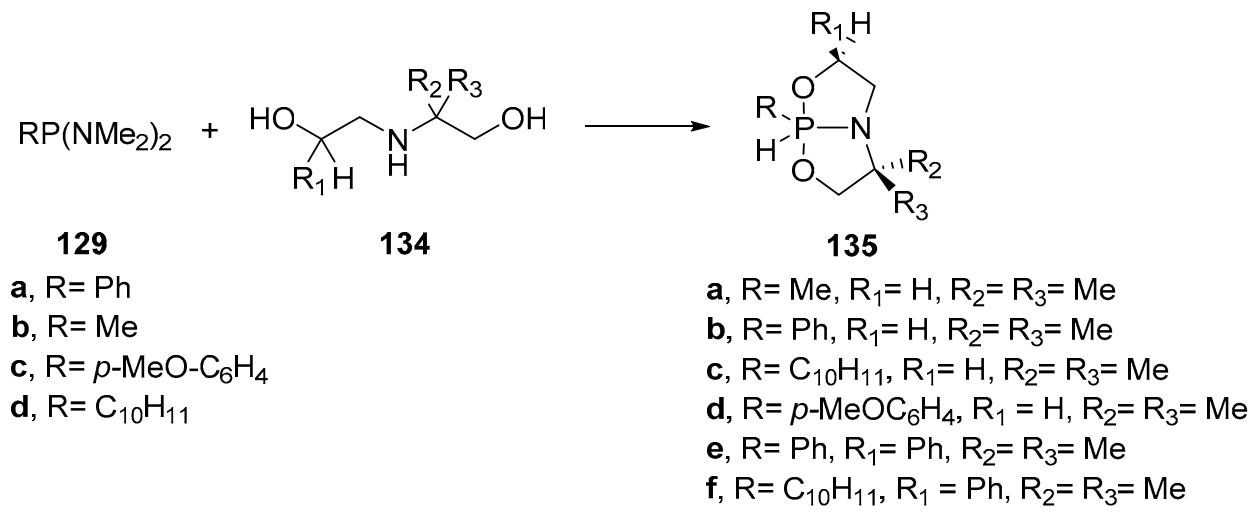

Scheme 55. Synthesis of bicyclic azaphosphoranes 135.

\section{P-2N-2O-1H and 10P-2N-3O Phosphoranes}

Chiral hydrospirophosphoranes derived from $L$-amino acids were for the first time obtained by Koenig et al. as early as 1979 [110]. More recently this approach based on the reaction of phosphorus trichloride with amino acids was used by Zhao and coworkers for the preparation of spirophosphoranes from enantiomerically pure valine 136a, isoleucine $\mathbf{1 3 6} \mathbf{b}$ and phenylalanine 136c. [111-114]. The spirophosphoranes formed as mixtures of diastereoisomers were separated to the pure stereoisomers 137a-c and 138a-c by reverse-phase HPLC or recrystallization (Scheme 56). 


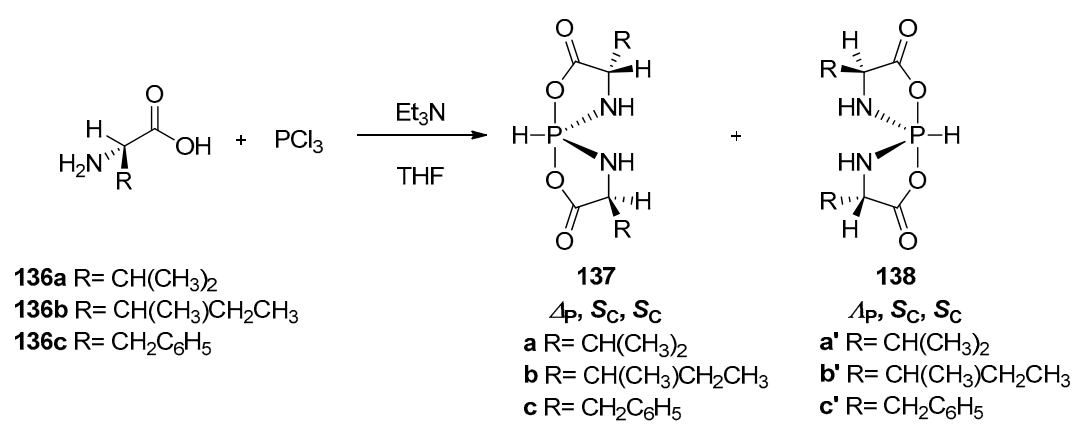

Scheme 56. Synthesis of hydrospirophosphoranes 137 and 138 derived from L-amino acids.

The pure stereoisomers 137 were used for the preparation of pentacoordinate spirophosphorane carbamates 139 [115] via the two-steps Atherton-Todd-type reaction (Scheme 57). Initially, the spirophosphoranes 137 reacted with $\mathrm{CCl}_{4}$ yielding chlorospirophosphoranes 140 (with retention of configuration at the phosphorus atom). In a second step chlorophosphoranes, upon the reaction with carbamate anions, (formed in situ from $\mathrm{CO}_{2}$ and secondary amines), afforded the carbamates $\mathbf{1 3 9}$ with inversion of configuration (Scheme 58).

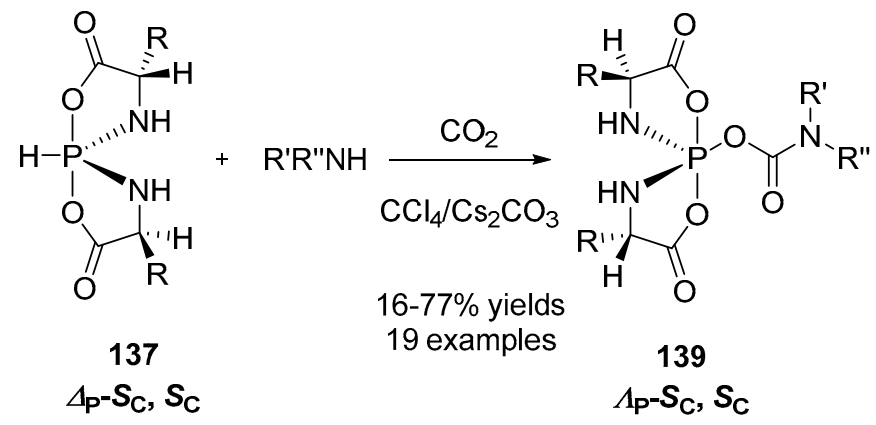

Scheme 57. Preparation of pentacoordinate spirophosphorane carbamates 139.<smiles>[R]C(N[PH+]1OC(=O)C([R])NC1C(=O)O)C(=O)O</smiles>

137 $\Delta_{\mathrm{p}}-S_{\mathrm{C}}, S_{\mathrm{C}}$<smiles>[R]C1NP2(Cl)(N[C@@H]1CC)OC(=O)[C@H]([R])O2</smiles>

140 $\Delta \mathrm{p}-S_{\mathrm{C}}, S_{\mathrm{C}}$

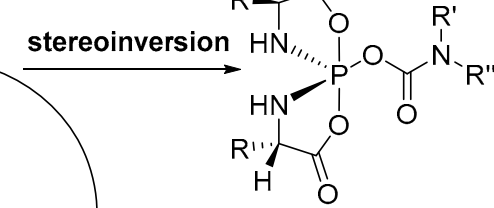

139 $\Lambda_{\mathrm{P}}-\mathrm{S}_{\mathrm{C}}, \mathrm{S}_{\mathrm{C}}$

Scheme 58. The mechanism of formation spirophosphorane carbamates 139.

Similarly, the spirophosphoranes 137a-e and 138a-e were used as substrates for the synthesis of pentacoordinate pyrospirophosphoranes containing a P-O-P bond 141-143 under modified Atherton-Todd conditions [116] (Scheme 59). It was found, upon optimization of the reaction conditions, that the spirophosphoranes $\mathbf{1 3 7} \mathbf{a}, \mathbf{b}$ gave the pure diasteroisomer of pyrospirophosphoranes $\mathbf{1 4 2} \mathbf{a}, \mathbf{b}$ whereas a mixture of diastereoisomers 141c-e and 143c-e were isolated if hydrospirophosphoranes $137 \mathbf{c}-\mathbf{e}$ were used as substrates. 

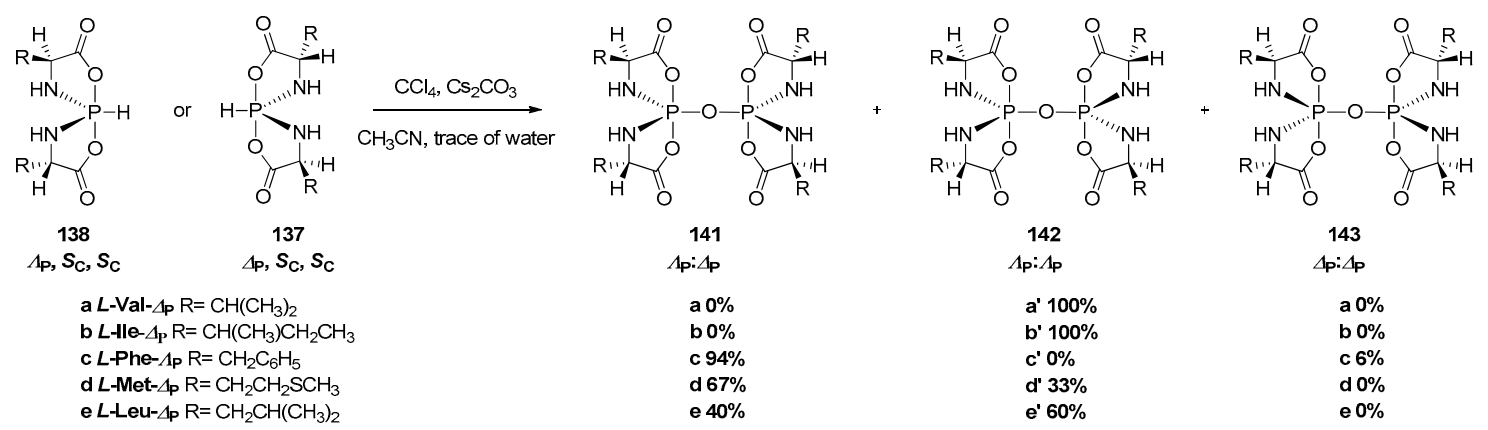

Scheme 59. Synthesis of pentacoordinate pyrospirophosphoranes 141-143.

A series of the alkoxy spirophosphoranes 144,145 was also prepared by this approach (Scheme 60) but unfortunately as a mixture of diastereoisomers (in most cases in 1:1 ratio) [117].<smiles>[R]C([R])C(=O)O[PH+]1N[C@@H]([R])C(=O)O1</smiles>

137 a $\mathrm{R}=\mathrm{CH}\left(\mathrm{CH}_{3}\right)_{2}$

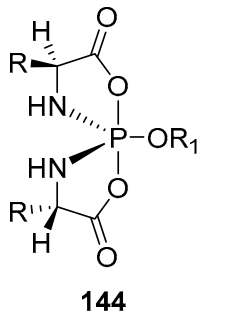

$\Lambda_{\mathrm{p}}, \mathrm{S}_{\mathrm{C}}, \mathrm{S}_{\mathrm{C}}$

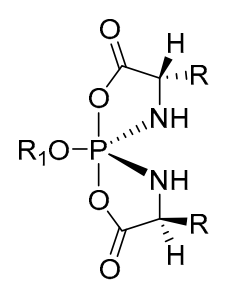

145

$\Delta_{\mathrm{p}}, \mathrm{S}_{\mathrm{C}}, \mathrm{S}_{\mathrm{C}}$

$\mathrm{R}_{1}=\mathrm{Me}, \mathrm{R}=\mathrm{CH}\left(\mathrm{CH}_{3}\right)_{2} ; \mathrm{dr}\left(\Lambda_{\mathrm{P}}: \Delta_{\mathrm{P}}\right)=50: 50$ yield $18 / 25 \%$

$\mathrm{R}_{1}=\mathrm{Et}, \mathrm{R}=\mathrm{CH}\left(\mathrm{CH}_{3}\right)_{2} ; \operatorname{dr}\left(\Lambda_{\mathrm{p}}: \Delta_{\mathrm{P}}\right)=44: 56$ yield $21 / 46 \%$

$\mathrm{R}_{1}=\mathrm{Et}, \mathrm{R}=\mathrm{CH}\left(\mathrm{CH}_{3}\right) \mathrm{CH}_{2} \mathrm{CH}_{3} ; \mathrm{dr}\left(\Lambda_{\mathrm{P}}: \Delta_{\mathrm{P}}\right)=44: 56$ yield $14 / 23 \%$

Scheme 60. Synthesis of alkoxy spirophosphoranes 144,145.

Bicyclic hydrophosphoranes 147 and $\mathbf{1 4 8}$ were synthesized as a 3:2 mixture of two epimers by the reaction of tris-(N,N-diethyl)phosphorus amide with isoleucinol 146. On the other hand, the tricyclic phosphorane 149 was isolated as a single stereoisomer in a similar reaction with the diaminodiole 151 (Scheme 61) [118]. The isolated spirophosphoranes 147-149 were used as chiral ligands in the Pd-catalyzed alkylation of 1,3-diphenyl allyl acetate with acceptable stereoselectivity (up to $74 \% e e$ ) (Scheme 61) [119]. Moreover, these hydrophosphorane derivatives were used to obtain complexes with $\left[\mathrm{Pt}(\mathrm{COD}) \mathrm{Cl}_{2}\right],\left[\mathrm{Rh}(\mathrm{CO})_{2} \mathrm{Cl}_{2}\left[\mathrm{Rh}(\mathrm{THF})_{2}(\mathrm{COD})\right]^{+} \mathrm{BF}_{4}{ }^{-}\right.$.

Chiral triquinphosphoranes 152-154 were easily synthesized from chiral enantiopure diaminodiols with a $C_{2}$ symmetry axis. It was shown that their structure is best represented by two trigonal bipyramid structures (TBP) with opposite absolute configurations at the phosphorus atom, $R_{\mathrm{P}}$ and $S_{\mathrm{P}}$, being in a fast equilibrium by a Berry pseudorotation process (Scheme 62) [120]. They reacted with various activated carbonyl compounds: trifluoroacetophenone, ketopantolactone and aromatic aldehydes to afford two diastereomeric hydroxyphosphoranes with a de up to $90 \%$ depending on the nature of the electrophile (Scheme 62). Diastereomeric mixture of hydroxyphosphoranes formed by the addition of ketopantolactone 155-156d-f were quantitatively converted into alkoxyphosphoranes 157 with diastereomeric excesses decreasing from $86 \%$ to $8 \%$ for $\mathrm{R}=\mathrm{Me}$, from $90 \%$ to $50 \%$ for $\mathrm{R}=\mathrm{iPr}$ and from $84 \%$ to $2 \%$ for $\mathrm{R}=\mathrm{Bn}$. 


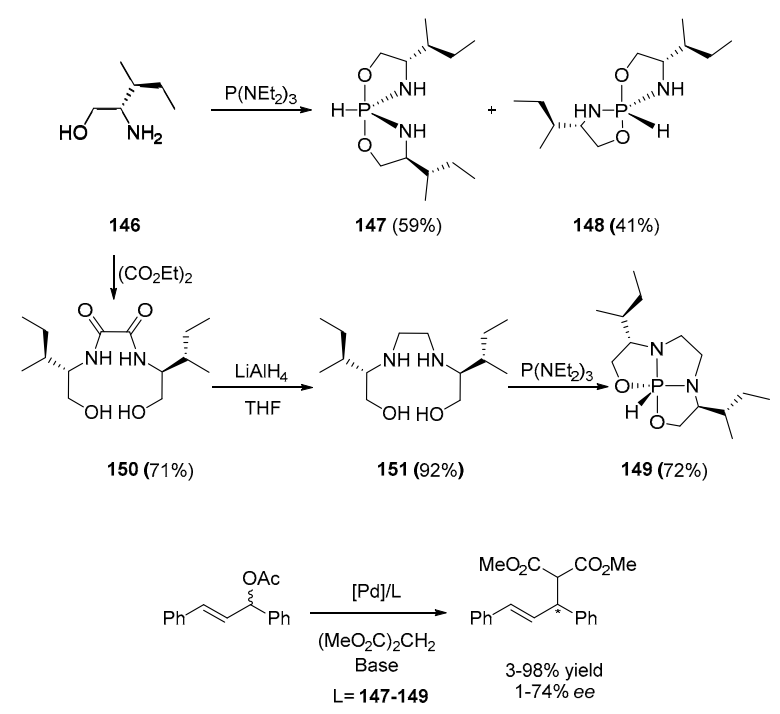

Scheme 61. Syntheses of bicyclic hydrophosphoranes 147-149 and their use as chiral ligands.
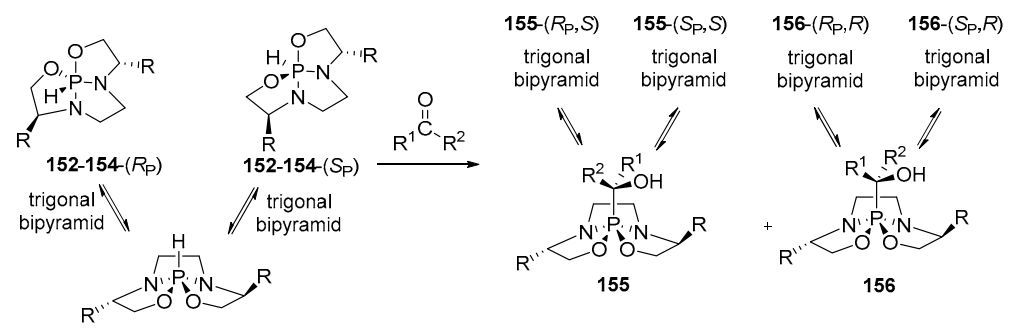

152-154 (square pyramid)

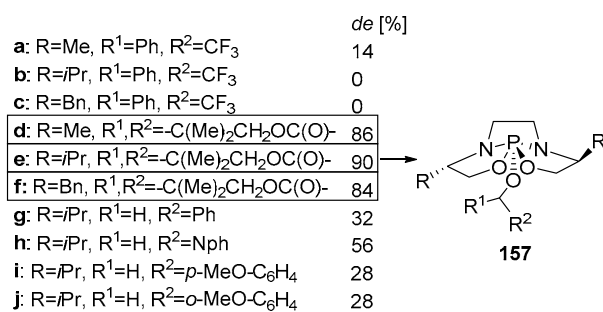

Scheme 62. Chiral triquinphosphoranes 152-154 and their reactions with various activated carbonyl compounds.

Similarly, the parent triquinphosphorane 158 was found to react with methyl, n-butyl, or $t$-butyl disulfide to produce the corresponding alkylthiophosphoranes $159 \mathbf{a}-\mathbf{c}$, although $t$-butylthio-phosphorane 159c was only a minor product and the major one was identified as the thiophosphoramide 160 (Scheme 63). Under irradiation at $-50{ }^{\circ} \mathrm{C} t$-butylthiophosphorane $159 \mathrm{c}$ was produced in high yield, however, by increasing the reaction temperature its slow conversion to 160 was observed. The reactivity of the triquinphosphorane $\mathbf{1 5 8}$ with alkyl disulfides was compared both under irradiation and in the dark at room temperature. The reactivity increased significantly in the case of $n$-butyl disulfide and $t$-butyl disulfide when the reactions were conducted under irradiation. This observation led to the conclusion that this type of the reaction does involve free radical species [121]. 


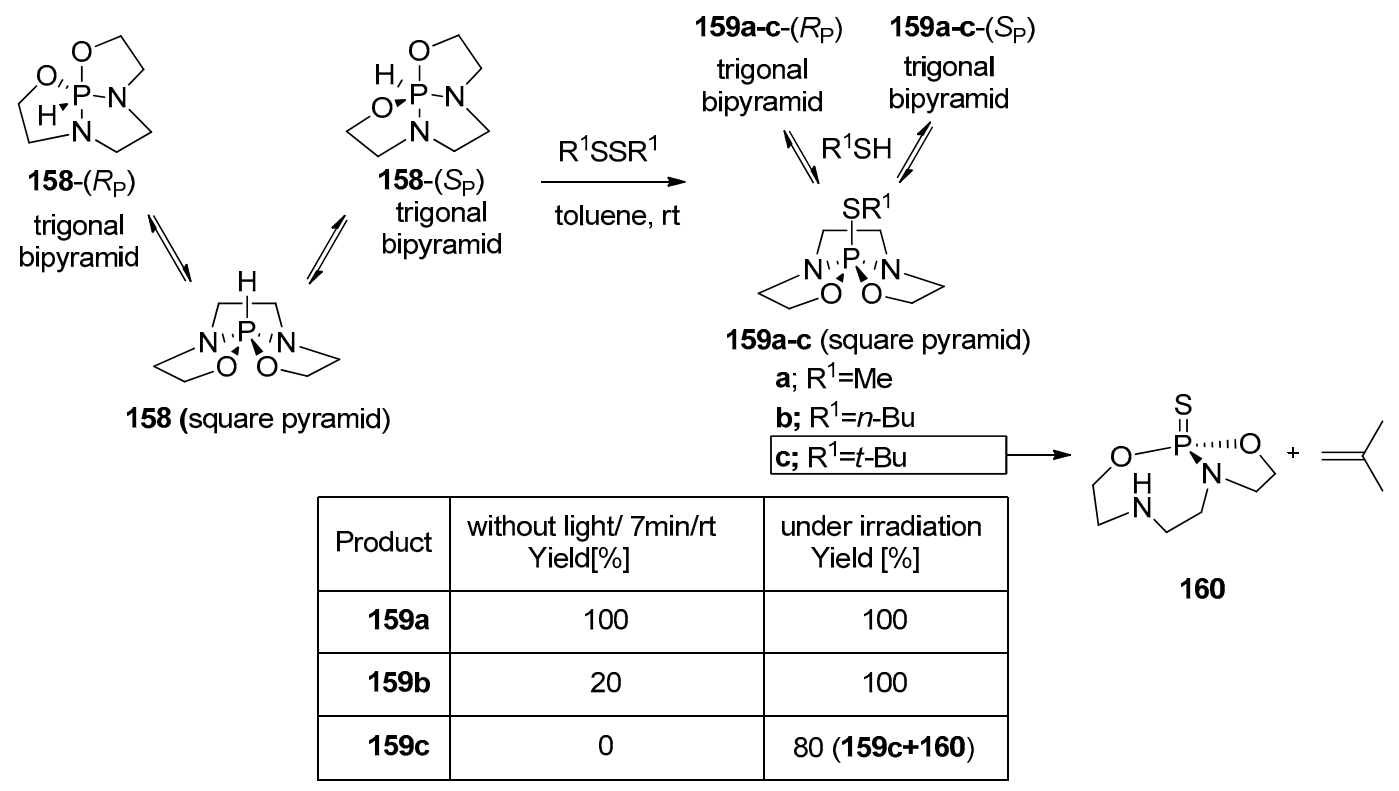

Scheme 63. Reaction of chiral hydrido-triquinphosphorane 158 with alkyl disulfides.

\section{P-1N-3O-1H and 10P-2N-2O-1H Phosphoranes}

Three synthetic protocols were applied to the preparation of this type of hydrophosphoranes with the use of amidooxime derivatives as substrates [122]. The first procedure started with the reaction of amidooxime 161a-e with $\mathrm{PCl}_{3}$ leading to 5-chloro-1,2,4,5-oxadiazaphospholines 162a-e. Their reactions with ethylene glycol or ortho-hydroxyphenol afforded the unsymmetrical spirophosphoranes 163 or 164 whereas the treatment with another dose of the amidooxime 161 led to symmetrically substituted spirophosphoranes 165a-e (Scheme 64).
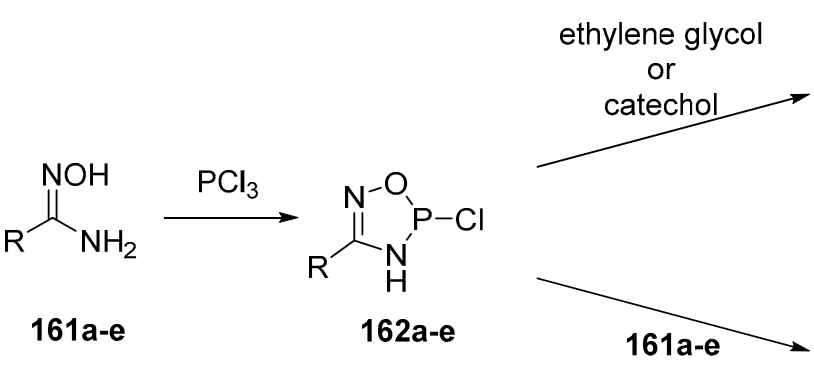

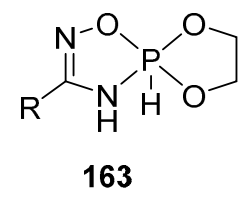

$(\mathrm{R}=\mathrm{Ph})$<smiles>[R]C1=NO[PH]2(N1)NC([R])=NO2</smiles>

165a-e
a, $\mathrm{R}=\mathrm{Me}$
b, $\mathrm{R}=i-\mathrm{Pr}$
c, $\mathrm{R}=t-\mathrm{Bu}$;
d, $\mathrm{R}=\mathrm{Bn}$
e, $\mathrm{R}=\mathrm{Ph}$

or<smiles></smiles>

$(\mathrm{R}=i-\operatorname{Pr}$ or $\mathrm{R}=\mathrm{Bn})$

Scheme 64. Syntheses of bicyclic hydrophosphoranes 163-165.

In the second approach, amidooxime $161 \mathrm{a}, \mathrm{c}, \mathrm{d}$ upon reaction with 2-dimethylamino1,3,2-dioxaphospholane 166 provided unsymmetrical spirophosphoranes $163 \mathbf{a}, \mathbf{c}, \mathbf{d}$ and in the reaction with 2-dimethylamino-(4,5)benzo-1,3,2-dioxaphospholane 167 in acetonitrile yielded unexpected symmetrically substituted spirophosphoranes 165a-e instead of unsymmetrically substituted phosphorane 164a-e (Scheme 65). 


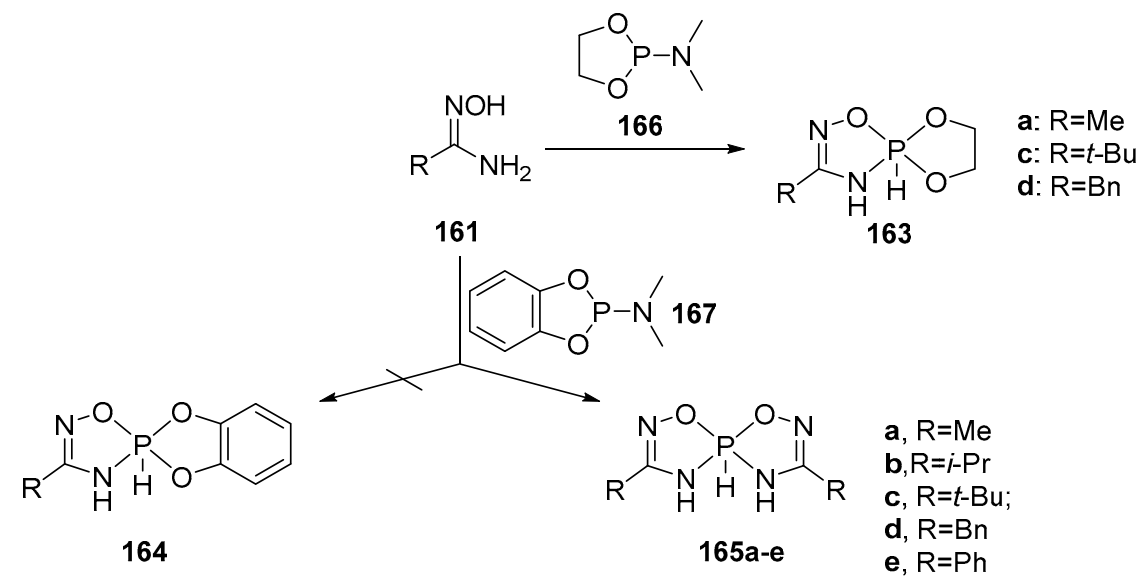

Scheme 65. Syntheses of bicyclic hydrophosphoranes 163 and 165.

The third synthetic pathway involved the treatment of amidooximes $161 \mathbf{b}, \mathbf{d}$,e with 2-chloro$(4,5)$ benzo-1,3,2-dioxaphospholane 168 leading, as it was expected, to the corresponding unsymmetrical spirophosphoranes 164. The latter were converted into the corresponding unsymmetrical spirophosphorane 170 by transesterification with pinacol 169 taking place in the presence of triethylamine (Scheme 66).

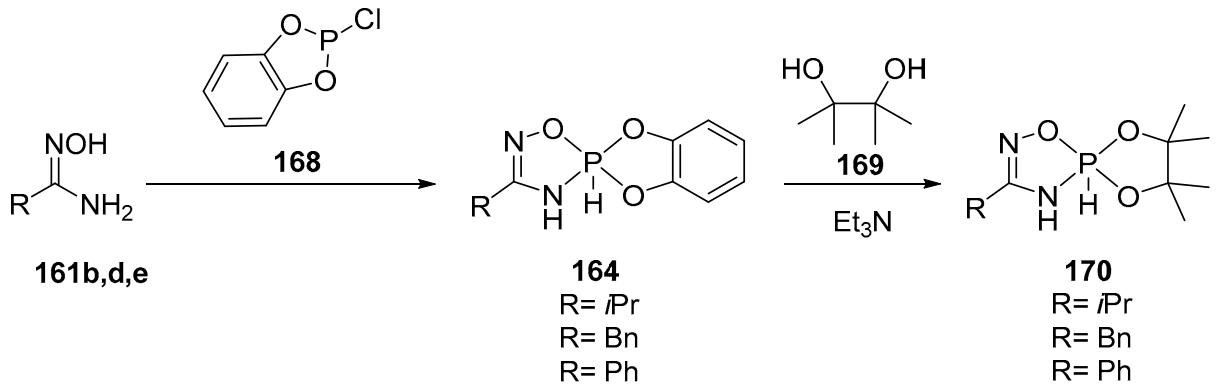

Scheme 66. Synthesis of bicyclic hydrophosphoranes 164 and 170.

10P-2N-3O and 10P-1N-4O Phosphoranes

Amidooximes 161d,e were used also as substrates for the preparation of the spirophosphoranes containing the $\mathrm{P}-\mathrm{OMe}$ grouping $\mathbf{1 7 3}, \mathbf{1 7 5}$. They were isolated upon application of the oxidation procedure carried out under basic conditions with iodine as an oxidant [122] (Scheme 67).
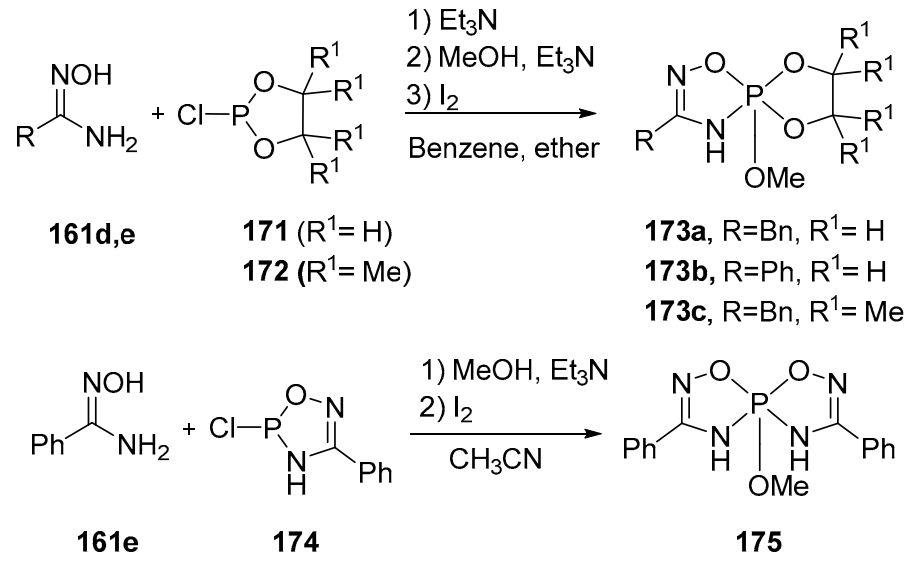

Scheme 67. Synthesis of bicyclic spirophosphoranes 173 and 175. 
$O$-Trimethylsilyl derivatives of spirophosphoranes 177 containing an amino acid residue were formed by the cyclisation of the corresponding cyclic amides 176 [123] (Scheme 68). The resulting spirophosphoranes $\mathbf{1 7 7}$ were used for the formation of peptides from amino acids such as histidine, serine, threonine and $\alpha$-alanine but not $\beta$-alanine.

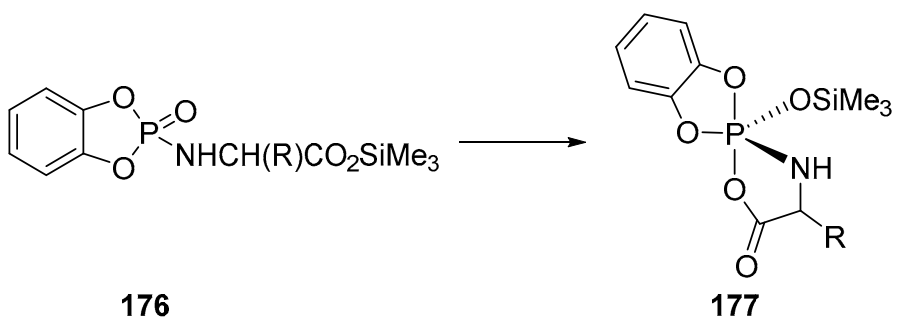

Scheme 68. Synthesis of bicyclic phosphoranes 177.

Later on, spirophosphoranes $\mathbf{1 7 7}$ were also used as substrates in a new method for the solid phase synthesis of oligopeptides. This approach was based on their rapid reaction with hydroxymethyl polystyrene resin leading to unstable hydroxyphosphoranes $\mathbf{1 7 8}$ which after mild hydrolysis gave the phosphate $\mathbf{1 8 0}$ and a solid phase bound amino-acids 179. Coupling of $\mathbf{1 7 9}$ with further phosphorane 177, followed by hydrolysis afforded a solid phase bound dipeptide. The process was repeated to give oligopeptides. The final oligopeptide was liberated from the resin by treatment with TFA [124] (Scheme 69).

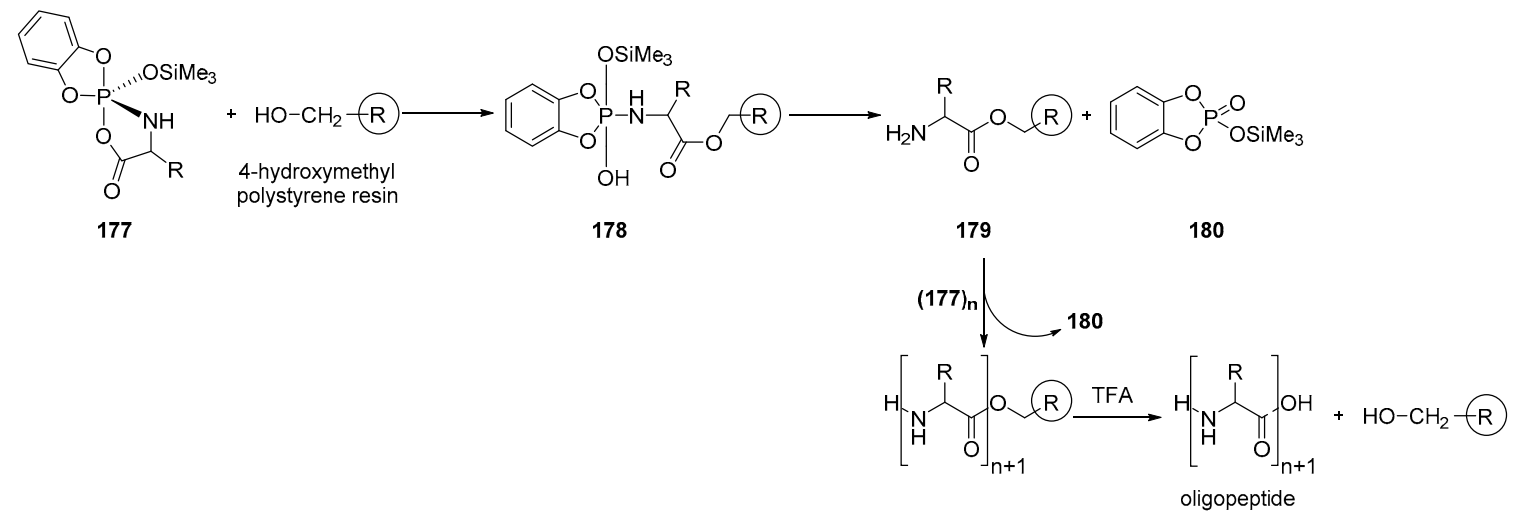

Scheme 69. Synthesis of oligopeptides with the use of spirophosphoranes 177 as substrates.

\section{P-4O-1H Phosphoranes}

The synthesis and reactivity of the hydrophosphorane $\mathbf{1 8 1}$ derived from perfluoropinacol was described as early as 1983 [125]. It was found that it could be rapidly oxidized by dimethyl sulfoxide to form the hydroxyphosphorane 182, and then silylated to the derivative $\mathbf{1 8 3}$. Chlorine and bromine reacted with 181 to give the corresponding halospirophosphoranes $\mathbf{1 8 4 a , b}$. In this context it is interesting to note that the corresponding fluorospirophosphorane $184 \mathrm{c}$ was obtained from the dichlorofluoro-perfluoropinacolophosphorane $\mathrm{FCl}_{2} \mathrm{P}\left(\mathrm{CH}_{2} \mathrm{Ph}\right)\left[\mathrm{OC}\left(\mathrm{CF}_{3}\right) \mathrm{C}\left(\mathrm{CF}_{3}\right) \mathrm{O}\right] 185$ and dilithium perfluoropinacolate $\mathrm{LiOC}\left(\mathrm{CF}_{3}\right) \mathrm{C}\left(\mathrm{CF}_{3}\right) \mathrm{OLi}$ 186. In the presence of triethylamine the hydrophosphorane 181 reacted with benzyl bromide or acetyl chloride to form the phosphoranes 187 or 188 . Moreover, the treatment of the hydrophosphorane 181 with trimethylphosphine gave a thermally unstable phosphonium salts 189. Hydrophosphorane $\mathbf{1 8 1}$ undergoes fast hydrolysis affording phosphorous acid and perfluoropinacol [125] (Scheme 70). 


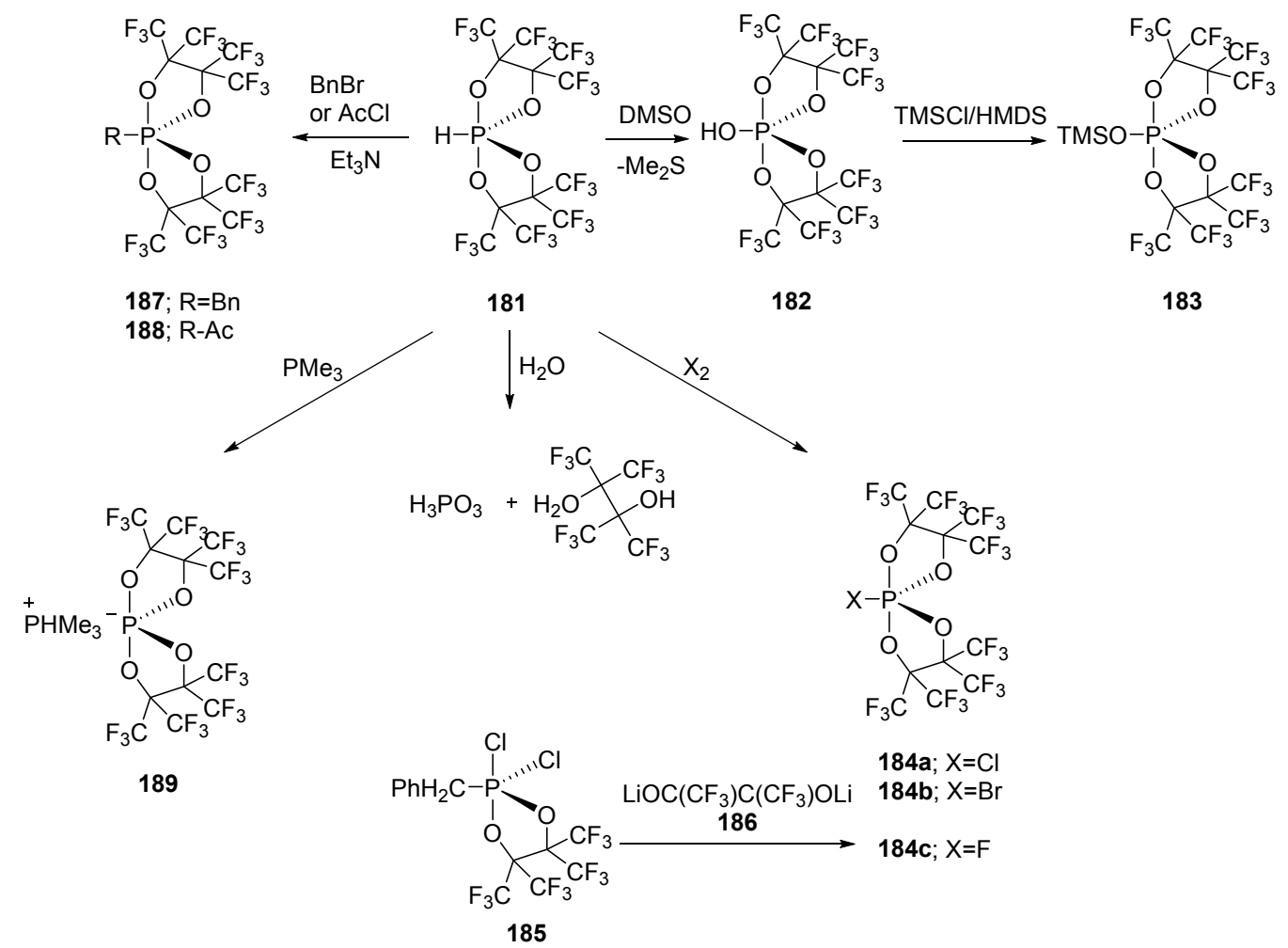

Scheme 70. Interconversions of spirophosphoranes 181 and 182.

10P-4O-1Metal and 10P-3N-1O-1Metal Phosphoranes

Another group of chiral pentacoordinate organophosphorus compounds are metallophosphoranes 192a-e. Nakazawa et al. [126] developed a synthetic method for the preparation of metallophosphoranes 192, which was based on the nucleophilic attack of the, in situ generated, anion 191a-e on the complexed trivalent phosphorus atom 190a,b (Scheme 71). For example, ferrocenyl complex 190a reacted with in situ generated anions 191a-e to form chiral metallophosphoranes 192a-e. The valency of the phosphorus atom increased from III to V without breaking the Fe-P bond and the nature of Fe-P bond changed from coordinative to covalent.

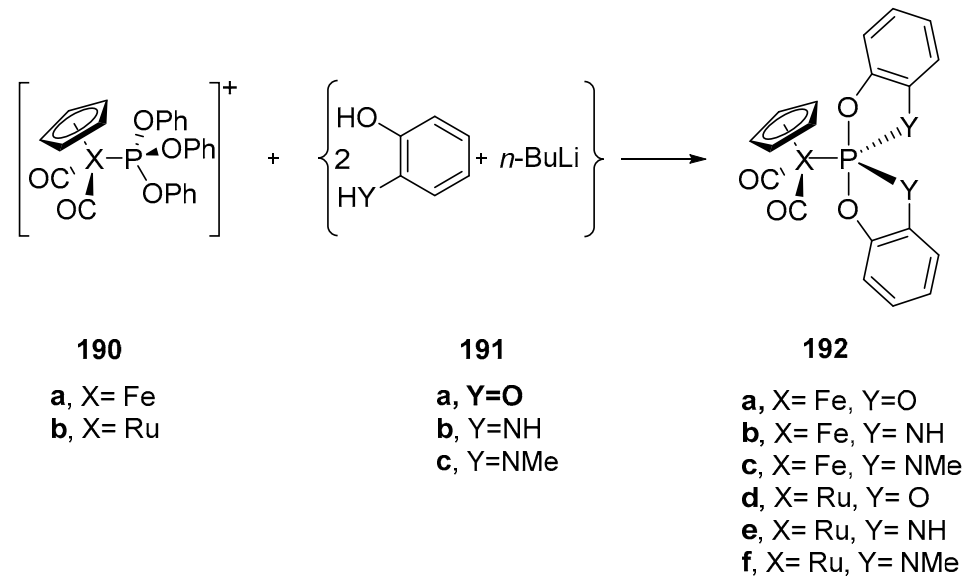

Scheme 71. Preparation of chiral metallophosphoranes 192.

It is worthy to note that treatment of $\mathrm{HP}\left(\mathrm{C}_{6} \mathrm{H}_{4} \mathrm{NH}\right)_{2}-193$ with $n \mathrm{BuLi}$ led to unexpected deprotonation on nitrogen atom (giving 194b) instead on phosphorus (to afford 194a). Moreover, 
an amide anion 194b is in an equilibrium with phosphoranide 194a. The reaction of the in situ generated anion 194a with $[\mathrm{Cp}(\mathrm{CO}) \mathrm{LFeCl}](195 \mathrm{a}$ or $195 \mathrm{~b})$ gave a P-metallated phosphorane 196a or 196b. On the contrary, the reaction the equilibrated mixture of 194 with $\left[\mathrm{Cp}(\mathrm{CO}) \mathrm{CoI}_{2}\right]-197$ led to $\mathrm{N}$-metallated compound 198. Interestingly, in the reaction of the phosphoranide 194a with MeI the P-methylated product 199 was formed. On the other hand, electrophiles such as $\mathrm{Me}_{3} \mathrm{SiCl}, \mathrm{Me}_{3} \mathrm{GeCl}$ or $\mathrm{Me}_{3} \mathrm{SnCl}$, provided $\mathrm{N}$-substituted products $(\mathbf{2 0 0 a}-\mathbf{c})[127,128]$ (Scheme 72).

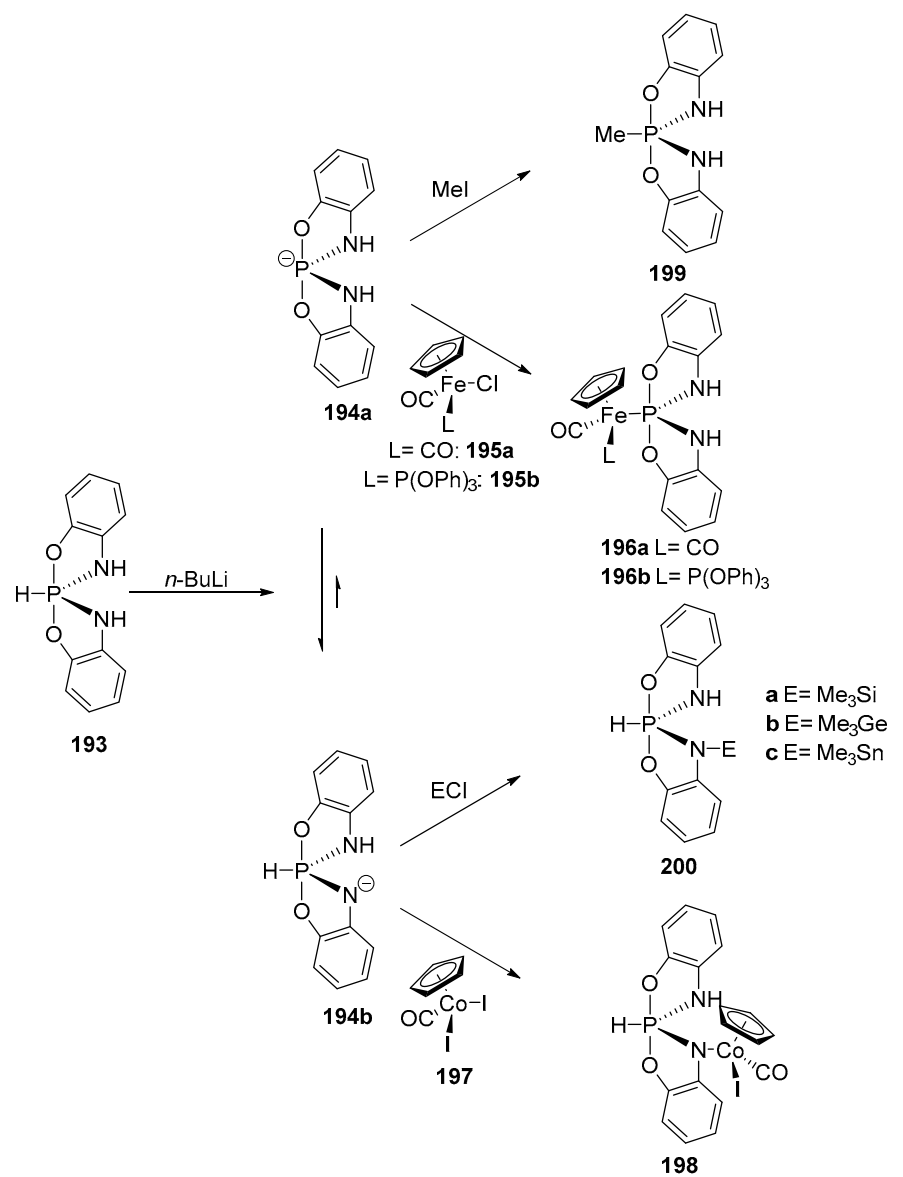

Scheme 72. Functionalization of hydrophosphorane 193 leading to phosphoranes 196 and 198-200.

Moreover, it was found that in the presence of a base a migration of pentacoordinate phosphorane fragment occured from the transition metal in $\mathbf{1 9 6}$ to the carbon atom in the $\mathrm{Cp}$ ring in 201 [129] (Scheme 73).

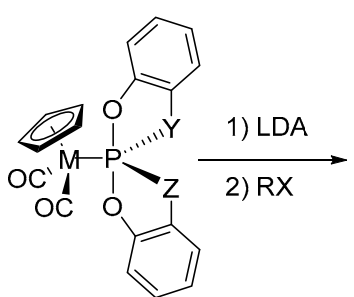

196

$\mathrm{M}=\mathrm{Fe}$ or $\mathrm{Ru}$

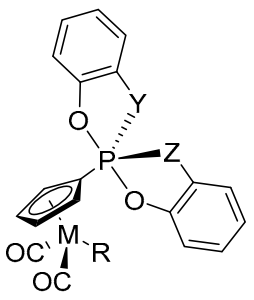

201

$\mathrm{Y}=\mathrm{NMe}$ or $\mathrm{O}$

$Z=\mathrm{NMe}$ or $\mathrm{O}$

$\mathrm{R}=\mathrm{Me}$ or $\mathrm{CH}_{2} \mathrm{Ph}$

Scheme 73. Alkylation of metallophosphoranes 196. 


\section{P-5O Phosphoranes}

The hydroxyphosphorane $\mathbf{2 0 3}$ was prepared by $\mathrm{N}_{2} \mathrm{O}_{4}$ oxidation of the parent hydrophosphorane 202 (Scheme 74). Its single crystal analysis showed an almost perfect trigonal bipyramidal structure with the unit cell containing two molecules of the same helicity connected by $\mathrm{H}$-bonds between the $\mathrm{P}-\mathrm{OH}$ and carbonyl groups [130].<smiles>O=C1CO[Pb]2(OCC(=O)O2)O1</smiles>

202

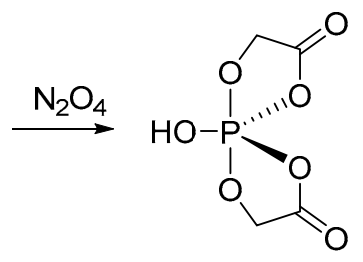

203

Scheme 74. Oxidation of hydrophosphorane 202.

The triethylammonium salt of the hydroxyspirophosphorane 205 derived from $n$-butyl tartrate was prepared on treatment of the very acidic $\left[\mathrm{p} K_{\mathrm{a}}=4.4(\mathrm{DMSO})\right]$ monocyclic phosphorus ester 204 with triethylamine (Scheme 75) [131].

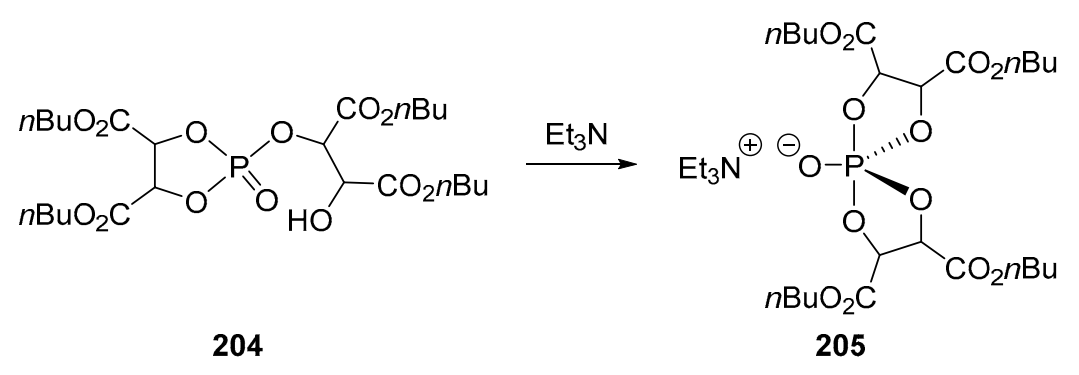

Scheme 75. Formation of salt 205.

The radical reaction of the bicyclic hydrophosphorane 206 derived from glycol with ethyl vinyl ether provided the corresponding $\mathrm{P}-\mathrm{C}$ spirophosphorane 207 along with the monocyclic phosphite 208. The reaction of the hydrospirophosphorane 206 with ethylene glycol and enamine 209 afforded the pentaoxyspirophosphorane $\mathbf{2 1 0}$ with the simultaneous reduction of the enamine 209 to amine 211 [132] (Scheme 76).

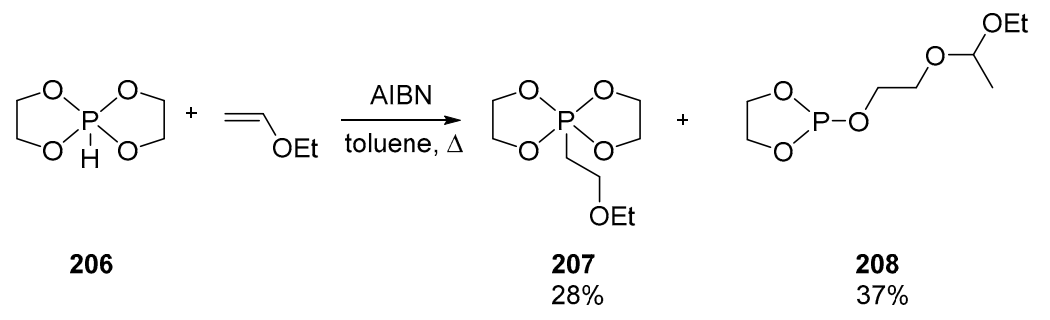

2<smiles>O=C1CO[PH]2(OCCO2)O1</smiles>

206<smiles>C1=CCCC(N2C[CH-]CC2)=C1</smiles>

209<smiles>OCCOC1CCCCC1</smiles>

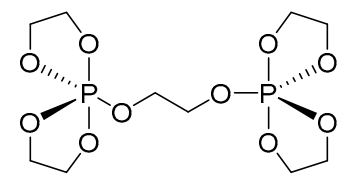

210

$100 \%$<smiles>C1CCC(N2CCCC2)C1</smiles>

211

Scheme 76. Synthesis of spirophosphoranes 207 and 210. 


\section{Conclusions}

Enantiomerically enriched pentavalent phosphoranes constitute an interesting group of heterocyclic chiral auxiliaries. Their still limited applications in asymmetric synthesis are however hampered mainly by a limited access to diastereomerically pure and especially enantiomerically pure (or at least enantiomerically enriched) samples. It is our hope that this review, which describes briefly the basic procedures used for the preparation of these derivatives, their stereoisomerization mechanisms and their selected interconversions, will encourage wider interest in the synthesis, structural determinations and applications of these groups of chiral, hypervalent organophosphorus derivatives.

Acknowledgments: Preparation of this review was financially supported by the National Science Center, Poland fund awarded based on the decisions (grant Maestro UMO-2012/06/A/ST5/00227 to (Józef Drabowicz).

Conflicts of Interest: The authors declare no conflict of interest.

\section{References}

1. Eliel, E.L.; Wilen, S.H. Stereochemistry of Organic Compounds; John Wiley \& Sons: New York, NY, USA, 1994.

2. Wolf, C. Dynamic Stereochemistry of Chiral Compounds: Principles and Applications; The Royal Society of Chemistry: Cambridge, UK, 2008.

3. Singh, J.; Hagen, T.J. Chirality and Biological Activity. In Burger's Medicinal Chemistry and Drug Discovery, 7th ed.; Abraham, D., Rotella, D., Eds.; John Wiley \& Sons: New York, NY, USA, 2010; pp. 127-166.

4. Reddy, I.K.; Mehvar, R. Chirality in Drug Design and Development; CRC Press: New York, NY, USA; Basel, Switzerland, 2004.

5. Mori, K. Bioactive Natural Products and Chirality. Chirality 2011, 23, 449-462. [CrossRef] [PubMed]

6. Ojima, I. (Ed.) Catalytic Asymmetric Synthesis, 3rd ed.; Wiley-VCH: New York, NY, USA, 2010.

7. Christmann, M.; Brase, S. (Eds.) Asymmetric Synthesis: More Methods and Applications; Wiley-VCH: New York, NY, USA, 2012.

8. Bruckner, R. Organic Mechanisms: Reactions, Stereochemistry and Synthesis; Harmata, M., Ed.; Springer: Berlin/Heidelberg, Germany, 2010.

9. Montchamp, J.-L. Phosphorus Chemistry I: Asymmetric Synthesis and Bioactive Compounds. In Topics in Current Chemistry; Springer International Publishing: Cham, Switzerland, 2015; Volume 360.

10. Dutartre, M.; Bayardon, J.; Juge, S. Applications and stereoselective syntheses of P-chirogenic phosphorus compounds. Chem. Soc. Rev. 2016, 45, 5771-5794. [CrossRef] [PubMed]

11. International Union of Pure and Applied Chemistry (IUPAC). Compendium of Chemical Terminology, 2nd ed.; (the "Gold Book"); McNaught, A.D., Wilkinson, A., Eds.; Blackwell Scientific Publications: Oxford, UK, 1997.

12. Quin, L.D. A Guide to Organophosphorus Chemistry; John Wiley \& Sons: New York, NY, USA, 2000.

13. Musher, J.I. The Chemistry of Hypervalent Molecules. Angew. Chem. Int. Ed. Engl. 1969, 8, 54-68. [CrossRef]

14. Perkins, C.W.; Martin, J.C.; Arduengo, A.J.; Lau, W.; Alergia, A.; Kochi, J.K. An Electrically Neutral sigma-Sulfuranyl Radical from the Homolysis of a Perester with Neighboring Sulfenyl Sulfur: 9-S-3 Species. J. Am. Chem. Soc. 1980, 102, 7753-7759. [CrossRef]

15. Hayes, R.A.; Martin, J.C. Organic Sulfur Chemistry: Theoretical and Experimental Advances; Bernardi, F., Csizmadia, J.G., Mangini, A., Eds.; Elsevier: Amsterdam, The Netherlands, 1985; Chapter 8; pp. 408-458.

16. Akiba, K. Hypervalent Compounds. In Chemistry of Hypervalent Compounds; Akiba, K., Ed.; Wiley-VCH: New York, NY, USA, 1999; Chapter 1; pp. 1-8.

17. Akiba, K. Structure and Reactivity of Hypervalent Organic Compounds: General Aspects. In Chemistry of Hypervalent Compounds; Akiba, K., Ed.; Wiley-VCH: New York, NY, USA, 1999; Chapter 2; pp. 9-47.

18. Noury, S.; Silyi, B.; Gillespie, J.R. Chemical Bonding in Hypervalent Molecules: Is the Octet Rule Relevant? Inorg. Chem. 2002, 41, 2164-2172. [CrossRef] [PubMed]

19. Mutterties, E.L.; Schunn, R.A. Pentacoordination. Q. Rev. 1966, 20, 245-299.

20. Berry, R.S. Correlation of Rates of Intramolecular Tunneling Processes, with Application to Some Group V Compounds. J. Chem. Phys. 1960, 32, 933-938. [CrossRef]

21. Berry, J.S. Time-Dependent Measurements and Molecular Structure: Ozone. Rev. Mod. Phys. 1960, 32, 447-454. [CrossRef]

22. Sheppard, W.A. Alkyl- and Arylsulfur Trifluorides. J. Am. Chem. Soc. 1962, 84, 3058-3063. [CrossRef] 
23. Ugi, I.; Marquarding, D.; Klusacek, H.; Gillespie, P.; Ramirez, F. Berry Pseudorotation and Turnstile Rotation. Acc. Chem. Res. 1971, 4, 288-296. [CrossRef]

24. Gillespie, P.; Hoffmann, P.; Klusacek, H.; Marquarding, D.; Pfohl, S.; Ramirez, F.; Tsolis, E.A.; Ugi, I. Bewegliche Molekülgerüste-Pseudorotation und Turnstile-Rotation Pentakoordinierter Phosphorverbindungen und Verwandte Vorgänge. Angew. Chem. 1971, 83, 691-721. [CrossRef]

25. Ugi, I.; Marquading, D.; Klusacek, H.; Gokel, G.; Gillespie, P. Chemie und Logische Strukturen. Angew. Chem. 1970, 82, 741-771. [CrossRef]

26. Ramirez, F.; Ugi, I. Turnstile Rearrangement and Pseudorotation in the Permutational Isomerization of Pentavalent Phosphorus Compounds. Adv. Phys. Org. Chem. 1971, 9, 25-126.

27. Couzijn, E.P.A.; Slootweg, J.C.; Ehlers, A.W.; Lammertsma, K. Stereomutation of Pentavalent Compounds: Validating the Berry Pseudorotation, Redressing Ugi's Turnstile Rotation, and Revealing the Two- and Three-Arm Turnstiles. J. Am. Chem. Soc. 2010, 132, 18127-18140. [CrossRef] [PubMed]

28. Wittig, G.; Rieber, M. Darstellung und Eigenschaften des Pentaphenyl-phosphors. Justus Liebigs Ann. Chem. 1949, 562, 187-192. [CrossRef]

29. Ramirez, F. Condensations of carbonyl compounds with phosphite esters. Pure Appl. Chem. 1964, 9, 337-370. [CrossRef]

30. Ramirez, F. Recent developments in the chemistry of hydroxyphosphoranes. Bull. Soc. Chem. Fr. 1966, 8 , 2443-2450.

31. Westheimer, F.H. Pseudo-rotation in the hydrolysis of phosphate esters. Acc. Chem. Res. 1968, 1, 70-78. [CrossRef]

32. Hall, C.D. Pentacoordinated and hexacoordinated compounds. In Organophosphorus Chemistry; Hutchinson, D.W., Walker, B.J., Eds.; The Royal Society of Chemistry: London, UK, 1986; Volume 16, pp. 51-78.

33. Hall, C.D. Pentacoordinated and hexacoordinated compounds. In Organophosphorus Chemistry; Walker, B.J., Hobbs, J.B., Eds.; The Royal Society of Chemistry: London, UK, 1988; Volume 19, pp. 47-69.

34. Hall, C.D. Pentacoordinated and hexacoordinated compounds. In Organophosphorus Chemistry; Walker, B.J., Hobbs, J.B., Eds.; The Royal Society of Chemistry: London, UK, 1991; Volume 21, pp. 51-72.

35. Hall, C.D. Pentacoordinated and hexacoordinated compounds. In Organophosphorus Chemistry; Allen, D.W., Tebby, J.C., Eds.; The Royal Society of Chemistry: London, UK, 2003; Volume 33, pp. 68-83.

36. Hall, C.D. Pentacoordinated and hexacoordinated compounds. In Organophosphorus Chemistry; Allen, D.W., Tebby, J.C., Eds.; The Royal Society of Chemistry: London, UK, 2006; Volume 35, pp. 265-303.

37. Tebby, J.C. Pentacoordinated and hexacoordinated compounds. In Organophosphorus Chemistry; Allen, D.W., Tebby, J.C., Eds.; The Royal Society of Chemistry: London, UK, 2007; Volume 36, pp. 184-196.

38. Roschenthaler, G.-V. Pentacoordinated and hexacoordinated compounds. In Organophosphorus Chemistry; Allen, D.W., Tebby, J.C., Eds.; The Royal Society of Chemistry: London, UK, 2009; Volume 38, pp. 318-331.

39. Pajkert, R.; Roschenthaler, G.-V. Pentacoordinated and hexacoordinated compounds. In Organophosphorus Chemistry; Allen, D.W., Tebby, J.C., Loakes, D., Eds.; The Royal Society of Chemistry: London, UK, 2010; Volume 39, pp. 290-307.

40. Pajkert, R.; Roschenthaler, G.-V. Pentacoordinated and hexacoordinated compounds. In Organophosphorus Chemistry; Allen, D.W., Tebby, J.C., Loakes, D., Eds.; The Royal Society of Chemistry: London, UK, 2011; Volume 40, pp. 297-315.

41. Pajkert, R.; Roschenthaler, G.-V. Pentacoordinated and hexacoordinated compounds. In Organophosphorus Chemistry; Allen, D.W., Tebby, J.C., Loakes, D., Eds.; The Royal Society of Chemistry: London, UK, 2012; Volume 41, pp. 322-348.

42. Pajkert, R.; Roschenthaler, G.-V. Pentacoordinated and hexacoordinated compounds. In Organophosphorus Chemistry; Allen, D.W., Tebby, J.C., Loakes, D., Eds.; The Royal Society of Chemistry: London, UK, 2013; Volume 42, pp. 197-215.

43. Pajkert, R.; Roschenthaler, G.-V. Pentacoordinated and hexacoordinated compounds. In Organophosphorus Chemistry; Allen, D.W., Tebby, J.C., Loakes, D., Eds.; The Royal Society of Chemistry: London, UK, 2014; Volume 43, pp. 348-365.

44. Kolodiazhnyi, O.I. Recent developments in the asymmetric synthesis of $P$-chiral phosphorus compounds. Tetrahedron Asymmetry 2012, 23, 1-46. [CrossRef]

45. Randić, M. Symmetry properties of graphs of interest in chemistry. II. Desargues Levi graph Quantum Chemistry. Int. J. Quantum Chem. 1979, 15, 663-682. [CrossRef] 
46. DeBruin, K.E.; Naumann, K.; Zon, G.; Mislow, K. Topological representation of the stereochemistry of displacement reactions at phosphorus in phosphonium salts and cognate systems. J. Am. Chem. Soc. 1969, 91, 7031-7040. [CrossRef]

47. Moulin, D.; Bago, S.; Bauduin, C.; Darcel, C.; Juge, S. Asymmetric synthesis of P-stereogenic o-hydroxyarylphosphine (borane) and phosphine-phosphinite ligands. Tetrahedron Asymmetry 2000, 11, 3939-3956. [CrossRef]

48. Uziel, J.; Stéphan, M.; Kaloun, El B.; Genet, J.P.; Juge, S. Regio- and stereochemistry of nucleophilic attack at the P-chiral center of a dioxaphospholane-borane complex: A model of study for the P-O bond cleavage. Bull. Soc.Chim. Fr. 1997, 134, 379-389.

49. Lopez-Cortina, S.; Basiulis, D.I.; Marsi, K.; Munoz-Hernandez, M.A.; Fernandez-Zertuche, M. Synthesis of New 1,3-Oxaphosphorinanium Salts. Stereochemistry of Hydroxide-Induced Displacement of Methoxide Ion. J. Org. Chem. 2005, 70, 7473-7478. [CrossRef] [PubMed]

50. Van Lier, J.J.C.; Hermans, R.J.M.; Buck, H.M. Evidence in support of tetraoxaspirophosphorane intermediates with a six-membered ring and $\mathrm{P}[\mathrm{sbnd}] \mathrm{H}$ bond in intramolecular transesterification reactions. Phosphorus Sulfur Silicon Relat. Elem. 1984, 19, 173-188. [CrossRef]

51. Boisdon, M.T.; Malavaud, C.; Mathis, F.; Barrans, J. Investigations of phosphoranes with P-H bond during the reaction of aminophosphine with alcohols. Tetrahedron Lett. 1977, 18, 3501-3505. [CrossRef]

52. Chung, F.-L.; Earl, R.A.; Townsend, L.B. The novel synthesis of a [6:5:6] linear isoguanosine type tricyclic nucleoside using carbonyl sulfide. Tetrahedron Lett. 1980, 21, 1599-1602. [CrossRef]

53. Lönnberg, T.; Laine, M. Phosphorane intermediate vs. leaving group stabilization by intramolecular hydrogen bonding in the cleavage of trinucleoside monophosphates: Implications for understanding catalysis by the large ribozymes. Org. Biomol. Chem. 2010, 8, 349-356. [CrossRef] [PubMed]

54. Binyamin, I.; Meidan-Shani, S.; Ashkenaz, N. Synthesis of $\gamma$-hydroxypropyl P-chirogenic $( \pm)$-phosphorus oxide derivatives by regioselective ring-opening of oxaphospholane 2-oxide precursors. Beilstein J. Org. Chem. 2015, 11, 1332-1339. [CrossRef] [PubMed]

55. Kolodyazhnyi, O.I. 2-Halo-1,2 $\lambda^{5}$-oxaphosphetanes. Zh. Obsh Kh. 1986, 56, 283-298.

56. Labaudiniere, L.; Burgada, R. Evolution thermique de vinylphosphoranes. Tetrahedron 1986, 42, $3521-3536$. [CrossRef]

57. Dieckbreder, U.; Roschenthaler, G.-V.; Kolomeitsev, A.A. P-bis(trifluoromethyl) ylides: Synthesis and reactions. Heteroat. Chem. 2002, 13, 650-653. [CrossRef]

58. Francke, R.; Roschenthaler, G.-V.; Di Giacomo, R.; Dakternieks, D. Oxidative addition von hexafluoraceton an die phosphor(III)-verbindungen $t \mathrm{BuP}(\mathrm{X}) \mathrm{NEt}_{2}\left(\mathrm{X}=\mathrm{F}, \mathrm{Cl}, \mathrm{OCH}\left(\mathrm{CF}_{3}\right)_{2}\right.$. Phosphorus Sulfur Silicon Relat. Elem. 1984, 20, 107-115. [CrossRef]

59. Yamamoto, Y.; Nakao, K.; Hashimoto, T.; Matsukawa, S.; Suzukawa, N.; Kojima, S.; Akiba, K.-Y. Crystallographic and NMR studies on species intermediate between haloalkoxyphosphoranes and alkoxyphosphonium halides. Heteroat. Chem. 2011, 22, 523-530. [CrossRef]

60. Sase, S.; Kano, N.; Kawashima, T. Novel Synthetic Method of Fluorophosphoranes by Fluoride Ion Abstraction from Tetrafluoroborate. Phosphorus Sulfur Silicon Relat. Elem. 2002, 177, 2041. [CrossRef]

61. Hellwinkel, D. Organic Phosphorus Chemistry; Kosolapoff, G.M., Maier, L., Eds.; Wiley Interscience: New York, NY, USA, 1973; Volume 3, pp. 186-339.

62. Burger, K. Organophosphorus Reagents in Organic Synthesis; Cadogan, J.I.G., Ed.; Academic Press: London, UK, 1979.

63. Holmes, R.R. Structure and Spectroscopy and Reaction Mechanisms in Pentacoordinated Phosphorus; ACS Monograph Series 175 and 176; American Chemical Society: Washington, DC, USA, 1980; Volume 1-2.

64. Husband, J.B.; McNab, H. The thermolysis of pentacoordinate phosphorus heterocycles. Phosphorus Sulfur Silicon Relat. Elem. 1984, 20, 207-230. [CrossRef]

65. Burgada, R.R.; Setton, R. The Chemistry of Organophosphorus Compounds; Hartley, F.R., Ed.; Wiley-Interscience: Chichester, UK, 1994; Volume 3, pp. 185-277.

66. Kawashima, T. Chemistry of Hypervalent Compounds; Akiba, K.-Y., Ed.; Wiley: New York, NY, USA, 1999.

67. Hellwinkel, D. Die Stereochemie organischer Derivate des fünf-und sechsbindigen Phosphors, II. Über ein erstes optisch aktives Pentaarylphosphoran. Chem. Ber. 1966, 99, 3642-3659. [CrossRef]

68. Monkowius, U.N.; Mitzel, W.; Schier, A.; Schmidbaur, H. 5-Organyl-5-phosphaspiro[4.4]nonanes: A Contribution to the Structural Chemistry of Spirocyclic Tetraalkylphosphonium Salts and Pentaalkylphosphoranes. J. Am. Chem. Soc. 2002, 124, 6126-6132. [CrossRef] [PubMed] 
69. Byrne, P.A.; Muldoon, J.; Ortin, Y.; Müller-Bunz, H.; Gilheany, D.G. Investigations on the Operation of Stereochemical Drift in the Wittig Reaction by NMR and Variable-Temperature NMR Spectroscopy of Oxaphosphetane Intermediates and Their Quench Products. Eur. J. Org. Chem. 2014, 86-98. [CrossRef]

70. Vedejs, E.; Meier, G.P.; Snoble, K.A.J. Low-temperature characterization of the intermediates in the Wittig reaction. J. Am. Chem. Soc. 1981, 103, 2823-2831. [CrossRef]

71. Kawashima, T.; Soda, T.; Okazaki, R. Synthesis, Structure, and Thermolysis of N-Apical 1,2 $\lambda^{5}$-Azaphosphetidines with a Pentacoordinate P Center and the First Observation of Their N-Equatorial Pseudorotamers. Angew. Chem. Int. Ed. Engl. 1996, 35, 1096-1098. [CrossRef]

72. Kawashima, T.; Kato, K.; Okazaki, R. Synthese, Struktur und Thermolyse eines 3-Methoxycarbonyl1,2 $\lambda^{5}$-oxaphosphetans. Angew. Chem. 1993, 105, 941-942. [CrossRef]

73. Kawashima, T.; Kato, K.; Okazaki, R. Synthesis, Structure, and Thermolysis of a 3-Methoxycarbonyl1,2 $\lambda^{5}$-oxaphosphetane. Angew. Chem. Int. Ed. Eng. 1993, 32, 869-870. [CrossRef]

74. Kano, N.; Kikuchi, A.; Kawashima, T. The first isolable pentacoordinate 1,2 $\lambda^{5}$-azaphosphetine: Synthesis, X-ray crystallographic analysis, and dynamic behaviour. Chem. Commun. 2001, 2096-2097. [CrossRef]

75. López, J.G.; Ramallal, A.M.; González, J.; Roces, L.; Garcia-Ganda, S.; Iglesias, M.J.; Oña-Burgos, P.; Ortis, F.L. Mechanisms of Stereomutation and Thermolysis of Spiro-1,2-oxaphosphetanes: New Insights into the Second Step of the Wittig Reaction. J. Am. Chem. Soc. 2012, 134, 19504-19507. [CrossRef] [PubMed]

76. García-López, J.; Peralta-Pérez, E.; Forcén-Acebal, A.; García-Granda, S.; López-Ortiz, F. Dilithiated phosphazenes: Scaffolds for the synthesis of olefins through a new class of bicyclic 1,2-oxaphosphetanes. Chem. Commun. 2003, 856-857. [CrossRef]

77. Kojima, S.; Kajiyama, K.; Akiba, K.-Y. Characterization of Enantiomeric Pairs of Optically Active 10-P-5 Phosphoranes with Asymmetry Only at Phosphorus. Bull. Chem. Soc. Jpn. 1995, 68, 1785-1797. [CrossRef]

78. Kojima, S.; Kajiyama, K.; Akiba, K.-Y. Characterization of an optically active pentacoordinate phosphorane with asymmetry only at phosphorus. Tetrahedron Lett. 1994, 35, 7037-7040. [CrossRef]

79. Kajiyama, K.; Yoshimune, M.; Nakamoto, M.; Matsukawa, S.; Kojima, S.; Akiba, K.-Y. Highly Selective One-Pot Synthesis of Spirophosphoranes Exhibiting Reversed Apicophilicity by Oxidation of Dianions Generated from P-H Spirophosphorane. Org. Lett. 2001, 3, 1873-1875. [CrossRef] [PubMed]

80. Akiba, K.-Y.; Matsukawa, S.; Kajiyama, K.; Nakamoto, M.; Kojima, S.; Yamamoto, Y. Novel results obtained by freezing berry pseudorotation of phosphoranes (10-P-5). Heteroat. Chem. 2002, 13, 390-396. [CrossRef]

81. Kajiyama, K.; Yoshimune, M.; Kojima, S.; Akiba, K.-Y. A New Method for the Formation of Anti-apicophilic (O-cis) Spirophosphoranes-Kinetic Studies on the Stereomutation of O-cis Arylspirophosphoranes to Their O-trans Isomers. Eur. J. Org. Chem. 2006, 2739-2746. [CrossRef]

82. Kano, N.; Miyaka, H.; Kawashima, T. Hypervalent Silicon and Phosphorus Atoms in Single Molecules: Synthesis and Properties of Phosphoranylalkoxysilicates and a Phosphoranyloxysilicate. Chem. Lett. 2007, 36, 1260-1261. [CrossRef]

83. Miyaka, H.; Kano, N.; Kawashima, T. Synthesis and Properties of Pentacoordinate Phosphorus Compounds Containing a Pentacoordinate Silicon Atom. Phosphorus Sulfur Silicon Relat. Elem. 2008, 183, 673-674. [CrossRef]

84. Jiang, X.-D.; Kakuda, K.; Matsukawa, S.; Yamamichi, H.; Kojima, S.; Yamamoto, Y. Synthesis and application of a bidentate ligand based on decafluoro-3-phenyl-3-pentanol: Steric effect of pentafluoroethyl groups on the stereomutation of O-equatorial C-apical spirophosphoranes. Chem. Asian J. 2007, 2, 314-323. [CrossRef] [PubMed]

85. Jiang, X.-D.; Matsukawa, S.; Kakuda, K.; Fukuzaki, Y.; Zhao, W-L.; Li, L.-S.; Shen, H.-B.; Kojima, S.; Yamamoto, Y. Efficient synthesis of tetradecafluoro-4-phenylheptan-4-ol by a Cannizzaro-type reaction and application of the alcohol as a bulky Martin ligand variant for a new anti-apicophilic phosphorane. Dalton Trans. 2010, 39, 9823-9829. [CrossRef] [PubMed]

86. Jiang, X.-D.; Matsukawa, S.; Yamamoto, Y. Synthesis, structure and isomerization of arylphosphoranes with anti-apicophilic bonding modes using a novel bidentate ligand with two C2F5 groups. Dalton Trans. 2008, 3678-3687. [CrossRef] [PubMed]

87. Jiang, X.D.; Matsukawa, S.; Yamamichi, H.; Yamamoto, Y. Some Reactions of O-Equatorial Spirophosphoranes Bearing the Bidentate Ligand Based on Decafluoro-3-phenyl-3-pentanol. Heterocycles 2007, 73, 805-824.

88. Kojima, S.; Sugino, M.; Matsukawa, S.; Nakamoto, M.; Akiba, K.-Y. First Isolation and Characterization of an Anti-Apicophilic Spirophosphorane Bearing an Oxaphosphetane Ring: A Model for the Possible Reactive Intermediate in the Wittig Reaction. J. Am. Chem. Soc. 2002, 124, 7674-7675. [CrossRef] [PubMed] 
89. Sase, S.; Kano, N.; Kawashima, T. Pentacoordinate 1H-Phosphirenes: Reactivity, Bonding Properties, and Substituent Effects on Their Structures and Thermal Stability. J. Org. Chem. 2006, 71, 5448-5456. [CrossRef] [PubMed]

90. Sase, S.; Kano, N.; Kawashima, T. Synthesis and Structure of the First 1,20 $\sigma^{5}$-Selenaphosphirane. J. Am. Chem. Soc. 2002, 124, 9706-9707. [CrossRef] [PubMed]

91. Sase, S.; Kano, N.; Kawashima, T. Synthesis of the First Stable Pentacoordinate Selenaphosphirane. Phosphorus Sulfur Silicon Relat. Elem. 2002, 177, 2039-2040. [CrossRef]

92. Sase, S.; Kano, N.; Kawashima, T. Isolation of a Cyclic Intermediate in the Reaction of a Phosphorus Ylide with Elemental Sulfur: Synthesis, Structure, and Reactivity of a 1,2 $\sigma^{5}$-Thiaphosphirane. Chem. Lett. 2004, 33, 1434-1435. [CrossRef]

93. Ken Hirotsu, K.; Akihiro Okamoto, A.; Kozo Toyota, K.; Masaaki Yoshifuji, M. X-ray structure of 3-diphenylmethylene-2-(2,4,6-tri-t-butylphenyl)thiaphosphirane 2-sulfide: The first thiaphosphirane with exo-methylene. Heteroat. Chem. 1990, 1, 251-254. [CrossRef]

94. Kano, N.; Xing, J.-H.; Kikuchi, A.; Kawa, S.; Kawashima, T. Synthesis of Four- and Five-Membered Heterocycles Derived from an Iminophosphorane. Phosphorus Sulfur Silicon Relat. Elem. 2002, 177, 1685-1687. [CrossRef]

95. Kano, N.; Xing, J.-H.; Kawa, S.; Kawashima, T. Synthesis, structure, and thermolysis of pentacoordinate 1,3,2 $\lambda^{5}$-oxazaphosphetidines: The intermediates of aza-Wittig reactions. Tetrahedron Lett. 2000, 41, 5237-5241. [CrossRef]

96. Kano, N.; Xing, J.-H.; Kikuchi, A.; Kawashima, T. Formation and X-ray crystallographic analysis of a 1,2 $\lambda^{5}$-oxaphosphol-5(2H)-one. Heteroat. Chem. 2001, 12, 282-286. [CrossRef]

97. Burnaeva, L.M.; Mironov, V.F.; Romanov, S.V.; Ivkova, G.A.; Shulaeva, I.L.; Konovalova, I.V. Reaction of 2-Phenyl-1,3,2-benzodioxaphosphinan-4-one with Diethyl Benzylidenemalonate. Russ. J. Gen. Chem. 2001, 71, 488-489. [CrossRef]

98. Adachi, T.; Matsukawa, S.; Nakamoto, M.; Kajiyama, K.; Kojima, S.; Yamamoto, Y.; Akiba, K.-Y.; Re, S.; Nagase, S. Experimental Determination of the $\mathrm{n}_{\mathrm{N}} \rightarrow \sigma_{\mathrm{P}-\mathrm{O}}^{*}$ Interaction Energy of $O$-Equatorial C-Apical Phosphoranes Bearing a Primary Amino Group. Inorg. Chem. 2006, 45, 7269-7277. [CrossRef] [PubMed]

99. Acher, F.; Juge, S.; Wakselman, M. Chiral bicyclic spirophosphoranes in an Arbuzov-type reaction. Tetrahedron 1987, 43, 3721-3728. [CrossRef]

100. Sevenard, D.V.; Kazimir, E.L.; Pashkevich, I.; Röschenthaler, G.-V. Polyfluoroacylcycloalkanones in reactions with selected phosphorus(III) compounds. Heteroat. Chem. 2002, 13, 97-107. [CrossRef]

101. Terent'eva, S.A.; Pudovik, M.A.; Gubaidullin, A.T.; Litvinov, I.A.; Pudovik, A.N. Synthesis and crystal and molecular structures of 2-diethylaminomethyl- and 2-chloromethyl-2,2'-spirobi[benzo-1,3,2-oxazaphospholines]. Russ. J. Gen. Chem. 2001, 71, 330-336. [CrossRef]

102. Terent'eva, S.A.; Pudovik, M.A.; Pudovik, A.N. Aminoalkylation of 2-substituted 4,5-benzo-1,3,2oxazaphospholanes. Zh. Obshch. Khim. 1987, 57, 496-499.

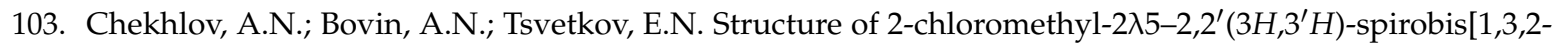
benzoxazaphosphole]. Izv. Akad. Nauk. SSSR Ser. Khim. 1991, 7, 1523-1526.

104. Terent'eva, S.A.; Nikolaeva, I.L.; Burilov, A.R.; Kharitonov, D.I.; Popova, E.V.; Pudovik, M.A.; Litvinov, I.A.; Gubaidullin, A.T.; Konovalov, A.I. Reaction of 2-Ethyl-1,3,2-bezoxaphospholine with Calix[4]resorcinarenes. Russ. J. Gen. Chem. 2001, 71, 389-395. [CrossRef]

105. Malavaud, C.; Barrans, J. Les phosphoranes monocycliques a ligand hydrogene intermediaires dans la synthese de spirophosphoranes. Tetrahedron Lett. 1975, 16, 3077-3080. [CrossRef]

106. Gholivand, K.; Mahzouni, H.R.; Molaei, F.; Kalateh, A.A. A phosphoryl to spiro-bicyclophosphorane transformation via $\beta$-amidic proton elimination in phosphorylated hydrazides. Tetrahedron Lett. 2012, 53, 5944-5947. [CrossRef]

107. Freytag, M.; Jones, P.G.; Schmutzler, R.; Yoshifuji, M. Reaction of (E)-bis-(2,4,6-tri-t-butylphenyl)diphosphane with tetrachloro-o-benzoquinone. Heteroat. Chem. 2001, 12, 300-308. [CrossRef]

108. Malavaud, C.; Charbonnel, Y.; Barrans, J. Synthese de P-methyl spirophosphoranes a partir de diols ou D-aminoalcohols. Tetrahedron Lett. 1975, 16, 497-498. [CrossRef]

109. Bonningue, C.; Brazier, J.F.; Houalla, D.; Osman, F.H. Phosphoranes par fermeture trans annulaire IV. Quelques Exemples de Phosphoranes Bicycliques Chiraux. Phosphorus Sulfur Silicon Relat. Elem. 1979, 5, 291-298. [CrossRef] 
110. Koenig, M.; Munoz, A.; Garrigues, B.; Wolf, R. Spirophosphoranes a Liaison P-H D' $\alpha$-Hydroxyacidies et de Tartrates D'Alcoyle: Preparation, Properties-Stereochimie. Phosphorous Sulfur Relat. Elem. 1979, 6, 435-451. [CrossRef]

111. Hou, J.-B.; Tang, G.; Guo, J.-N.; Liu, Y.; Zhang, H.; Zhao, Y.-F. Stereochemistry of chiral pentacoordinate spirophosphoranes correlated with solid-state circular dichroism and ${ }^{1} \mathrm{H}$ NMR spectroscopy. Tetrahedron Asymmetry 2009, 20, 1301-1307. [CrossRef]

112. Hou, J.-B.; Zhang, H.; Guo, J.-N.; Liu, Y.; Xu, P.-X.; Zhao, Y.-F.; Blackburn, G.M. Chirality at phosphorus in pentacoordinate spirophosphoranes: Stereochemistry by $\mathrm{X}$-ray structure and spectroscopic analysis. Org. Biomol. Chem. 2009, 7, 3020-3023. [CrossRef]

113. Yang, G.; Xu, Y.; Hou, J.; Zhang, H.; Zhao, Y. Determination of the Absolute Configuration of Pentacoordinate Chiral Phosphorus Compounds in Solution by Using Vibrational Circular Dichroism Spectroscopy and Density Functional Theory. Chem. Eur. J. 2010, 16, 2518-2527. [CrossRef] [PubMed]

114. Yang, G.; Xu, Y.; Hou, J.; Zhang, H.; Zhao, Y. Diastereomers of the pentacoordinate chiral phosphorus compounds in solution: Absolute configurations and predominant conformations. Dalton Trans. 2010, 39, 6953-6959. [CrossRef] [PubMed]

115. Cao, S.; Gao, P.; Gua, Y.; Zhao, H.; Wang, J.; Liu, Y.; Zhao, Y. Unexpected Insertion of $\mathrm{CO}_{2}$ into the Pentacoordinate P-N Bond: Atherton-Todd-Type Reaction of Hydrospirophosphorane with Amines. J. Org. Chem. 2013, 78, 11283-11293. [CrossRef] [PubMed]

116. You, X.H.; Qi, L.; Zheng, J.; Dai, W.; Guo, Y.C.; Zhao, Y.F.; Cao, S.X. Synthesis and Characterization of New Pyrospirophosphoranes Containing a P-O-P Bond by the Atherton-Todd Reaction. Heteroat. Chem. 2015, 26, 168-174. [CrossRef]

117. Dai, W.; Liu, Q.; You, X.; Zhou, Z.; Guo, Y.; Zhao, Y.; Cao, S. Synthesis and Characterization of Alkoxy Spirophosphoranes Prepared from Hydrospirophosphoranes and Sodium Alcoholates. Heteroat. Chem. 2016, 27, 63-71. [CrossRef]

118. Bondarev, O.G.; Mikhel, I.S.; Tsarev, P.V.; Petrovskii, P.V.; Davankov, V.A.; Gavrilov, K.N. Platinum and rhodium complexes with isoleucinol-based bi- and tricyclic hydrophosphoranes. Russ. Chem. Bull. Int. Ed. 2003, 52, 116-121. [CrossRef]

119. Gavrilov, K.N.; Polosukhin, A.I.; Bondarev, O.G.; Lyubimov, S.E.; Lyssenko, K.A.; Petrovski, P.; Davankov, V.A. Synthesis and properties of pentacoordinated phospha derivatives of iso-leucinol. A rare example of using of hydrophosphoranes as ligands in asymmetric catalysis. J. Mol. Catal. A Chem. 2003, 196, 39-53. [CrossRef]

120. Marchi, C.; Buono, G. Asymmetric Addition of Chiral Triquinphosphoranes on Activated Carbonyl Compounds. Tetrahedron Lett. 1999, 40, 9251-9254. [CrossRef]

121. Marchi, C.; Buono, G. Alkylthiylation of triquinphosphoranes by disulfides: An entry to chiral thiatriquinphosphoranes. Tetrahedron Lett. 2000, 41, 3073-3076. [CrossRef]

122. Lopez, L.; Fabas, C.; Barrans, J. Heterocycles phosphores derivant des amidoximes. II. Composes du phosphore penta et hexacoordine. Phosphorus Sulfur Silicon Relat. Elem. 1979, 7, 81-87. [CrossRef]

123. Zhao, Y.-F.; Han, B.; Chen, J.; Jiang, Y. Penta-Coordinate Phosphorus Compounds and Biochemistry. Phosphorus Sulfur Silicon Relat. Elem. 2002, 177, 1391-1396. [CrossRef]

124. Li, Z.; Fu, H.; Gong, H.; Zhao, Y. Convenient Solid Phase Synthesis of Oligopeptides Using Pentacoordinated Phosphoranes with Amino Acid Residue as Building Blocks. Bioorg. Chem. 2004, 32, 170-177. [CrossRef] [PubMed]

125. Schenthaler, G.-V.R.; Bohlen, R.; Storzer, W.; Sopchik, A.E.; Bentrude, W.G. Reaktionen eines Tetraalkoxyhydro-spirophosphorans. Z. Anorg. Allg. Chem. 1983, 507, 93-99. [CrossRef]

126. Nakazawa, H.; Kubo, K.; Miyoshi, K. Metallaphosphorane Chemistry: Preparations, Structures, and Reactivities. Bull. Chem. Soc. Jpn. 2001, 74, 2255-2267. [CrossRef]

127. Nakazawa, H.; Ogawa, T.; Kawamura, K.; Miyoshi, K. Syntheses of Metallaphosphorane Complexes and Berry Pseudorotation. Phosphorus Sulfur Silicon Relat. Elem. 2002, 177, 2163-2164. [CrossRef]

128. Nakazawa, H.; Kawamura, K.; Ogawa, T.; Myoshi, K. Syntheses, structure, and Berry pseudorotation of ruthenium-, iron-, and cobalt-phosphorane complexes. J. Organomet. Chem. 2002, 646, 204-211. [CrossRef]

129. Kubo, K.; Nakazawa, H.; Kawamura, K.; Mizuta, T.; Miyoshi, K. Migration Reaction of a Hypervalent Fragment: Base-Induced Migration of a Phosphorane Fragment from Iron to the Cyclopentadienyl Ring in $\mathrm{Cp}(\mathrm{CO}){ }_{2} \mathrm{Fe}\left\{\mathrm{P}\left(\mathrm{OC}_{6} \mathrm{H}_{4} \mathrm{Y}\right)\left(\mathrm{OC}_{6} \mathrm{H}_{4} \mathrm{Z}\right)\right\}(\mathrm{Y}, \mathrm{Z}=\mathrm{NMe}, \mathrm{NH}, \mathrm{O})$. J. Am. Chem. Soc. 1998, 120, 6715-6721. [CrossRef] 
130. Munoz, A.; Gornitzka, H. Phosphorus, Structure Cristalline D'un Hydroxyspirophosphorane Modele D'intermediaire de Reactions Impliquant Des Esters Cycliques de L'acide Phosphorique. Phosphorus Sulfur Silicon Relat. Elem. 2003, 178, 55-60. [CrossRef]

131. Boyer, D.G.; Boisdon, M.-T.; Rochal, A.; Munoz, A. Equilibre Ester Phosphorique, Hydroxyphosphorane Role des Liaisons Hydrogene, Acidite de Bronsted. Phosphorus Sulfur Silicon Relat. Elem. 2003, 178, 2117-2125. [CrossRef]

132. Laurenco, C.; Burgada, R. Mecanisme de formation et de transformation des spirophosphoranes-IX: Reactions des spirophosphoranes a liaison $\mathrm{P}-\mathrm{H}$ avec les ethers vinyliques et les enamines. Tetrahedron 1976, 22, 2253-2255. [CrossRef]

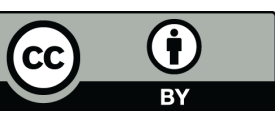

(C) 2016 by the authors; licensee MDPI, Basel, Switzerland. This article is an open access article distributed under the terms and conditions of the Creative Commons Attribution (CC-BY) license (http://creativecommons.org/licenses/by/4.0/). 